\title{
The effect of novel kappa opioid peptide receptor agonists on learning and memory in rats
}

\author{
Susan Adele Welsh
}

\section{VICTORIA UNIVERSITY OF WELLINGTON}

Te Whare Wananga o the Upoko o the Ika a Maui

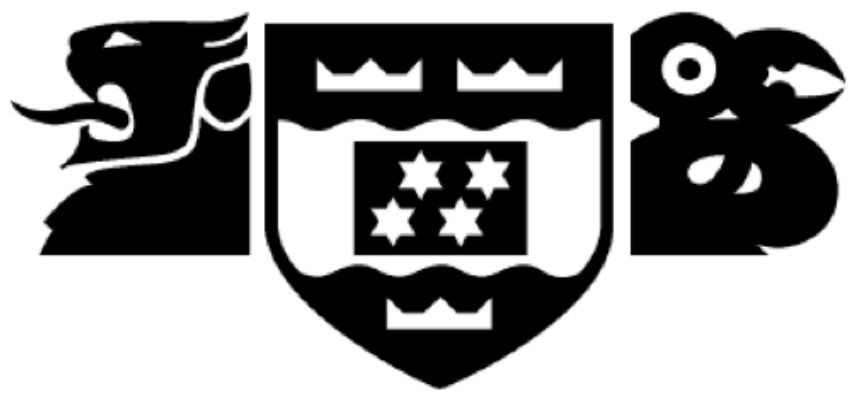

School of Biological Sciences

Te kura Matauranga Koiora

\section{A thesis}

submitted to the Victoria University of Wellington in fulfilment of the requirements of the degree of Master of Biomedical Science 


\section{Abstract}

Kappa opioid peptide receptors (KOPrs) are a class of opioid receptors which shown analgesic and anti-addictive properties. Nonaddictive analgesics would be beneficial as morphine, one of the most commonly prescribed opioids for chronic pain, activates the brain reward system and can lead to addiction. Although medical research is progressing rapidly, there is still no treatment for psychostimulant abuse. KOPr agonists show promise in this regard but display undesirable side effects and could negatively affect memory. Salvinorin A (Sal A), a structurally unusual KOPr agonist, has a reduced side effect profile compared to the more traditional KOPr agonists such as $\cup 50,488$. The effect of Sal A and $U 50,488$ on memory is controversial as they have both been shown to induce a memory impairment and also to improve memory impairments. Sal A also has a poor pharmacokinetic profile with a short duration of action. Structural analogues of Sal A have improved pharmacokinetic and side effect profiles compared to Sal A yet retain the analgesic and anti-addiction properties. This thesis will investigate whether Sal A analogues, namely Ethynyl Sal A (Ethy Sal A), Mesyl Salvinorin B (Mesyl Sal B), and Bromo Salvinorin A (Bromo Sal A), produce a memory impairment.

Male Sprague-Dawley rats were evaluated in the novel object recognition (NOR) task to determine whether novel Sal A analogues impair long term recognition memory. The degree of novelty was also investigated on a cellular basis through quantifying c-Fos immunoreactive neurons within the perirhinal cortex, an area of the brain shown to respond to novelty.

Acute administration of Sal A (0.3 and $1 \mathrm{mg} / \mathrm{kg})$ and novel analogues Ethy Sal A (0.3 and $1 \mathrm{mg} / \mathrm{kg}$ ), Mesyl Sal B (0.3 and $1 \mathrm{mg} / \mathrm{kg}$ ), and Bromo Sal A (1 mg/kg) showed no significant differences compared to vehicle when tested in the NOR task. The prototypical KOPr agonist, U50,488 (10 mg/kg), produced a significant decrease in recognition index compared to vehicle when tested in the same task as the novel analogues. Correlating the recognition indices calculated from $U 50,488$ in the NOR to c-Fos counts in the perirhinal cortex showed a strong positive correlation with an increase in $\mathrm{RI}$ relating to an increase in c-Fos activation. U50,488 (10 mg/kg) showed a non-significant trend 
compared to vehicle in the number of c-Fos immunoreactive cells within the perirhinal cortex.

Neither Sal A nor novel analogues affected NOR, suggesting no impairment of long term recognition memory. The lack of this side-effect, among others, demonstrates that the development of potent KOPr agonists with reduced side-effect profiles is feasible. These novel analogues show improvement over the traditional KOPr agonists. 


\section{Acknowledgements}

Had I attempted Master's without any help, we all know I wouldn't have gotten very far so l'd like to thank the the following people for helping me out and keeping me sane over the last couple of years.

Firstly, to my supervisor Dr Bronwyn Kivell. Thank you for providing me with this opportunity to experience what real science is first hand and allowing me to learn new and interesting skills along the way.

To everyone in my lab group, you've helped me through a lot of interesting and difficult times. To Aimee and David for teaching me everything about lil ratties from scratch and for showing me how amazing show tunes are when distractions are needed. To Kelly, for putting up with a lot of stupid questions from me and demolishing all the baking I brought in. To Amy for letting me distract you and helping me find work to do so I was being productive without doing my work. To the rest of the rat pack: Amy, Stephen, Diana, and Andy, thank you all for appreciating the cute little faces we get to work with every day. And to everyone else who's been a part of the lab with me: Adam, Phyllida, Jasleen, Miguel, Rickiii, Sam, Isobel, Afnan, and Harvir, good on ya for having a shot at sciencing as well.

To my other science buddies: Nikki for providing advice so I didn't have to start from scratch, Marie for showing me a skill l've greatly enjoyed learning, to Vimal for being a thesis saver and for sorting me out when I really needed it, to Jaime for going through this torture we call science with me, and to Cameron, for suffering with me from afar and even moreso for reading what was probably the most interesting thing you've ever picked up.

To the builders and fixers of things: Shaun, Neville, and Craig. Thank you for making all my objects for me and for fixing all the things I broke over the time I spent in the lab. Without you, I would have had to replace many things. To Derek and Sushila, the technicians who helped out with strange requests and always found a way to make my life slightly easier. To the SBS staff Mary, Charlotte, Lesley, Patricia, Mark, Stephen, and Paul. Thank you for showing me what to do and understanding what it was I really needed 
help with. To Peter Ritchie and Janet Pitman, thank you for letting me make use of your labs when I was unable to use mine so I could keep on keeping on.

A shout out to the crew at The Lab. Thank you for making my life slightly more bearable and not judging me too much with all the marshmallows I ate daily.

To Kate, Zoe, Kassie, Dale, Sean, Ali and Veronica, Struan, The White Lamington, a big thank you for helping me stay sane, for getting drunk with me, and for putting up with my craziness and grumpiness over the last years. Thanks for making sure I didn't die and made sure I was eating! Thank you for to Jean Goodman Dance Studios and Body Electric Pole Dance Studio for being a way to unwind and giving me space from science when I needed it. I'm so glad I know all of you because this year would have sucked a lot more if you weren't around

To my crazy massive family. Thanks for making me feel underqualified and giving me motivation to keep going and get through postgraduate. Thanks for being there for the random things I needed help with without much warning, and a super massive thanks for making sure I had food and was looking after myself over the last few months.

To all the animals I met along the way, thanks for making me happy and giving me fluffy snuggles.

And finally to Esther. You're pretty great. 


\section{Table of Contents}

Abstract

Acknowledgements iii

Table of Contents $\quad$ v

List of figures vii

List of tables vii

Abbreviations viii

1 Introduction 1

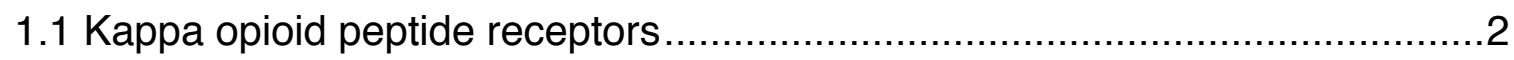

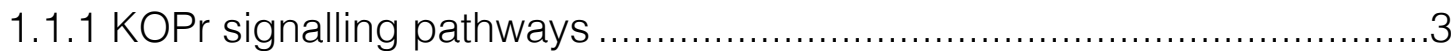

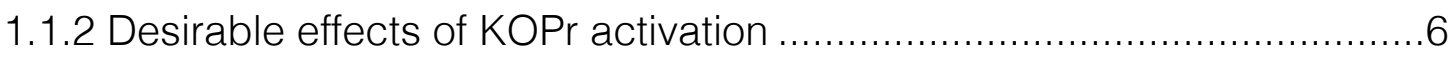

1.1.3 Undesirable KOPr side effects...............................................

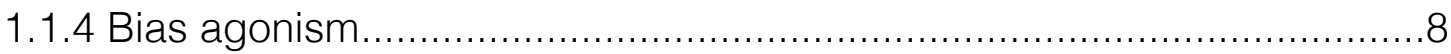

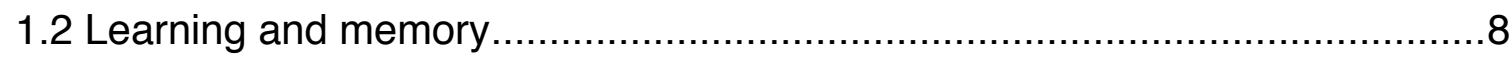

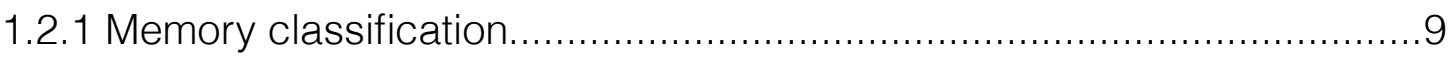

1.2.2 Brain regions involved in recognition memory ................................. 12

1.2.3 Animal models of learning and memory .................................. 15

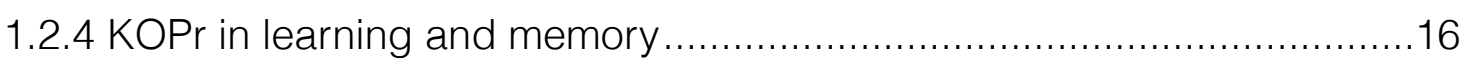

1.2.5 Immediate early genes in learning and memory ............................ 18

1.2.6 CREB in learning and memory ........................................... 19

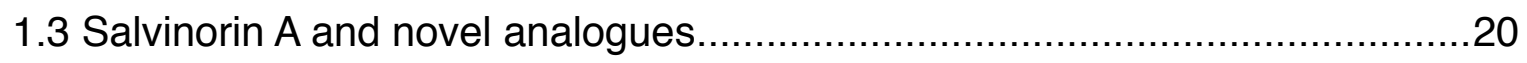

2 Aims and hypotheses $\quad 24$

3 Methods $\quad 25$

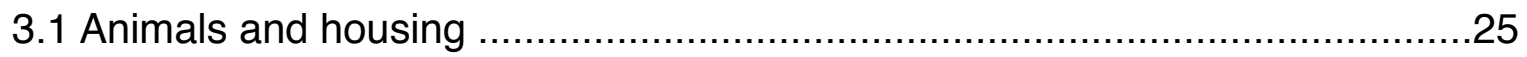

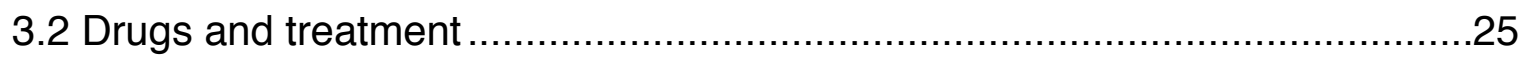

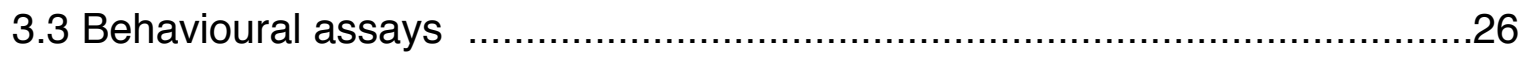

3.3.1 NOR task methods development ...............................................26

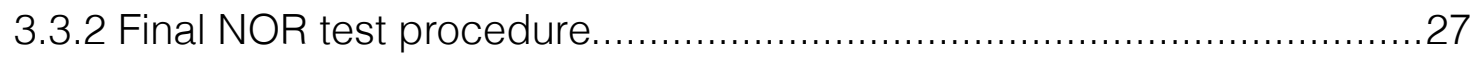

3.3.3 Forced swim test for immunohistochemistry methods development ......31 


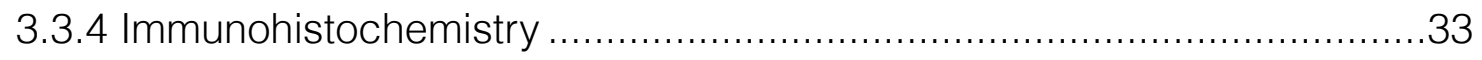

3.3.5 Quantification of c-Fos positive cells ...................................................33

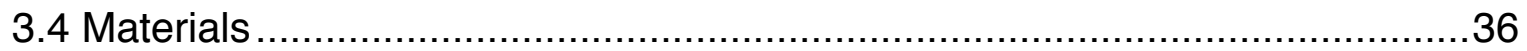

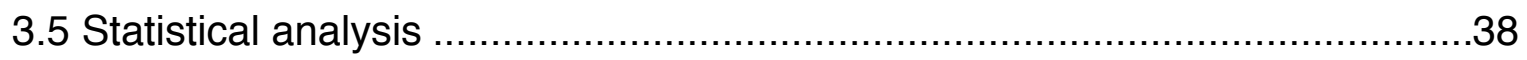

4 Results $\quad 39$

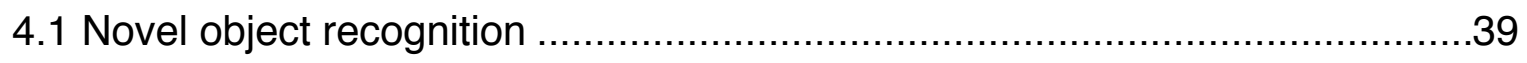

4.1.1 Larger objects allow for greater object exploration time ….................39

4.1.2 Increasing habituation time increases object exploration .....................39

4.1.3 Using a two-phase protocol increases the sensitivity of the positive

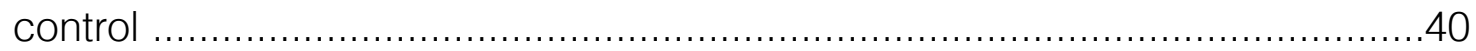

4.1.4 U50,488 causes a memory impairment in LTM but not STM ................42

4.1.5 Sal A analogues do not alter long term recognition memory .................44

4.1.6 The long term recognition memory impairment caused by $\mathrm{U} 50,488$ is

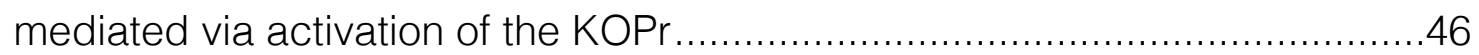

4.2 Activation of c-Fos in the perirhinal cortex correlates with U50,488 induced NOR

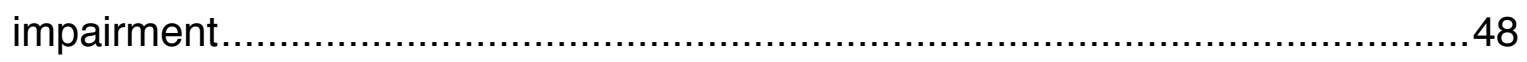

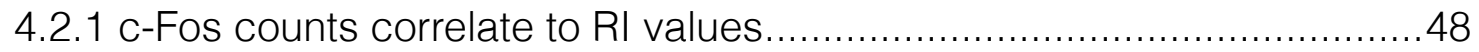

4.2.2 U50,488 treatment induces activation of c-Fos in the perirhinal cortex .48

\section{Discussion $\quad 50$}

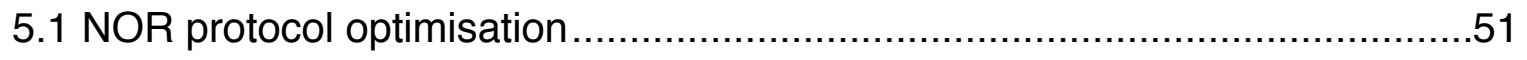

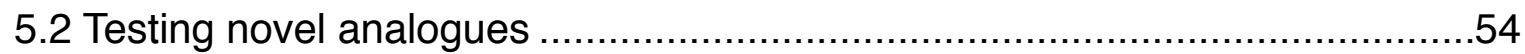

5.3 Possible mechanisms for memory impairment ..........................................5

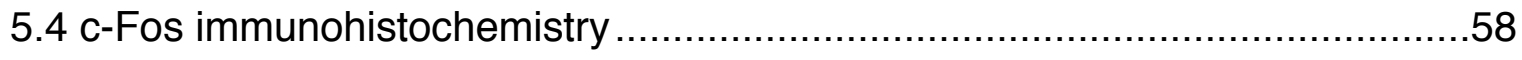

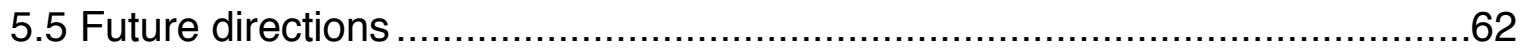

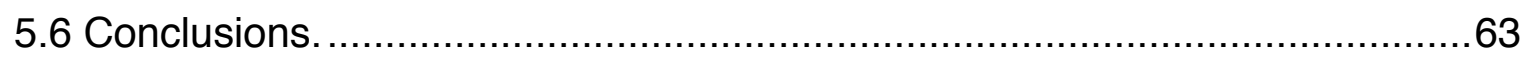

6 Supplementary information $\quad 64$

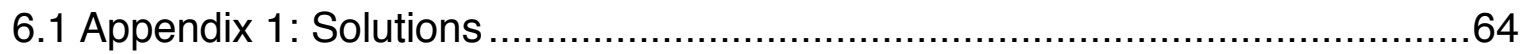

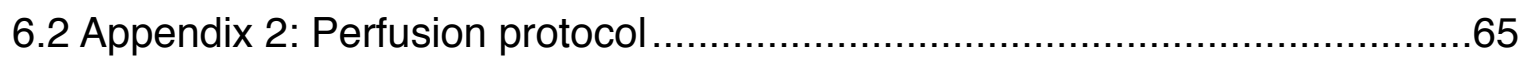

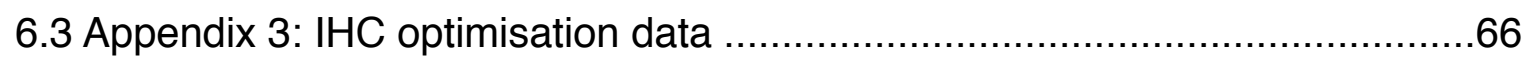

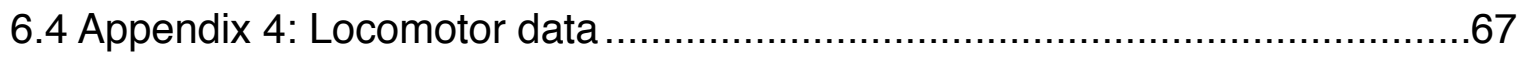

$\begin{array}{ll}7 \text { Literature cited } & 68\end{array}$ 


\section{List of figures}

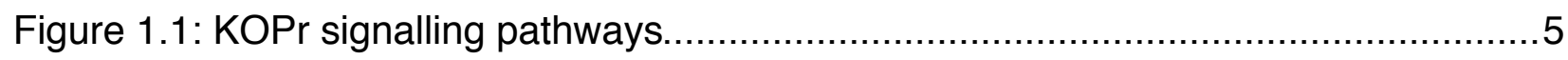

Figure 1.2: Learning and memory classification ...................................................11

Figure 1.3: Brain regions involved in learning and memory........................................14

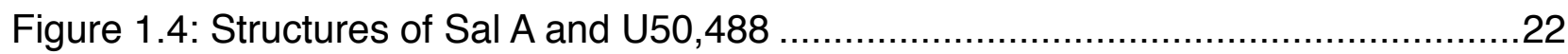

Figure 3.1: Visual representation of NOR methods trialled ........................................29

Figure 3.2: Rat brain regions used in forced swim stress..........................................32

Figure 3.3: Stereology method used when quantifying c-Fos positive cells .....................35

Figure 3.4: Objects used throughout the NOR tests......................................................37

Figure 4.1: Effects of Sal A on RI from the NOR test.............................................. 41

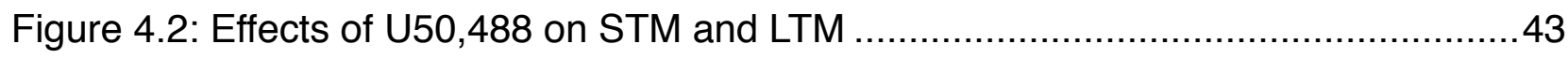

Figure 4.3: Effect of KOPr agonists on RI measured with the NOR task........................45

Figure 4.4: norBNI prevented U50,488 mediated long term recognition memory impairment

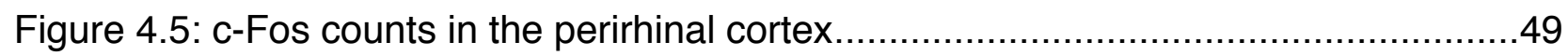

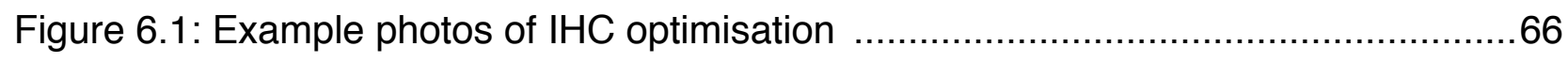

Figure 6.2: KOPr treatments affecting total distance travelled .....................................67

\section{List of tables}

Table 1.1: Pharmacokinetic properties of U50,488, Sal A, and novel analogues ...........23

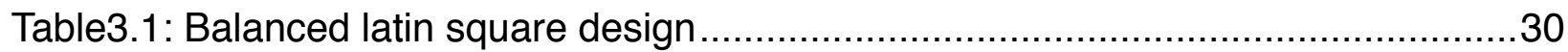




\section{Abbreviations}

$\beta$-THP: $\quad \beta$-tetrahydropyran Salvinorin B

Bromo Sal A: Bromo Salvinorin A

cAMP: $\quad$ Cyclic adenosine monophosphate

Cg: $\quad$ Cngulate cortex

CNS: $\quad$ Central nervous system

CRE: $\quad$ cAMP response element

CREB: $\quad$ CAMP response element binding protein

DAB: $\quad$ Diaminobenzidine

DOPr: Delta opioid peptide receptor

ERK1/2: $\quad$ Extracellular signal-regulated kinases 1 and 2

Ethy Sal A: Ethynyl Salvinorin A

FST: $\quad$ Forced swim test

GPCR: $\quad$ G-protein coupled receptor

GRK3: $\quad$ G-protein coupled receptor kinase 3

IHC: Immunohistochemistry

IL: $\quad$ Infralimbic cortex

ip: Intraperitoneal

ITI: $\quad$ Intertrial interval

JNK: $\quad c$-Jun $N$-terminal kinase

KOPr: $\quad$ Kappa opioid peptide receptor

LTM: $\quad$ Long term memory

LTP: $\quad$ Long term potentiation

Mesyl Sal B: Mesyl Salvinorin B

MOM Sal B: 2-Methoxymethyl Salvinorin B

MOPr: $\quad$ Mu opioid peptide receptor

NOR: $\quad$ Novel object recognition

norBNI: nor-Binaltorphimine

p38 MAPK: p-38 mitogen-activated protein kinase

PBS: $\quad$ Phosphate buffered saline 
PBS-T: $\quad$ Phosphate buffered saline with Triton X-100

PFA: $\quad$ Paraformaldehyde

PFC: $\quad$ Prefrontal cortex

PI3K: $\quad$ Phosphoinositide 3-kinase

PKCद: $\quad$ Protein kinase $\mathrm{C} \zeta$

Prh: $\quad$ Perirhinal cortex

RI: $\quad$ Recognition index

Sal A: $\quad$ Salvinorin A

sc: $\quad$ Subcutaneous

STM: $\quad$ Short term memory

U50,488: 2-(3,4-dichlorophenyl)-N-methyl-N-[(1R,2R)-2-pyrrolidin-1-ylcyclohexyl] acetamide

WM: $\quad$ Working memory 


\section{Introduction}

Kappa opioid peptide receptors (KOPrs) are a class of opioid receptors found throughout the central nervous system and periphery tissues. As opioid receptors, KOPr activation causes an analgesic effect (Aldrich \& McLaughlin, 2009). However, since activation of the KOPr system does not produce a rewarding effect (Wang, Sun, Tao, Chi, \& Liu, 2010), addiction to KOPr agonists is highly unlikely. The need for nonaddictive analgesics is high since pain is the leading cause for doctor and hospital visits in the United States. Chronic pain is reported to affect approximately 100 million American adults making it more prevalent than other diseases such as diabetes, coronary heart disease, strokes, and cancer (Institute of Medicine, 2011). One of the most commonly prescribed treatments for chronic pain is mu opioid peptide receptor (MOPr) agonist, morphine (Jordan, Cvejic, \& Devi, 2000). Unfortunately, the MOPr system activates the brain reward system. Addiction, a chronic relapsing brain disorder characterised by a compulsive, uncontrolled desire to take a drug (Koob \& Le Moal, 2001), is estimated to affect about $12 \%$ of New Zealand's population (National Committee for Addiction Treatment, 2016). Abuse of alcohol and drugs is the sixth highest contributor to disease within New Zealand (National Committee for Addiction Treatment, 2011) and comes at a high cost worldwide. In 2014, 60.9\% of recorded overdoses in the United States involved an opioid (Rudd, Seth, David, \& Scholl, 2016). Although medical research is progressing rapidly, there is still no FDA approved pharmaceutical treatment for psychostimulant abuse.

A novel KOPr agonist isolated from the naturally hallucinogenic plant Salvia divinorim, Salvinorin A (Sal A), has a reduced side effects compared to more traditional KOPr agonists but has a poor pharmacokinetic profile. Structural analogues of this compound show promise as potential future therapies both as nonaddictive analgesics and as anti-addiction therapies. This thesis will investigate whether these Sal A analogues produce a memory impairment as a side effect. 


\subsection{Kappa opioid peptide receptors}

The kappa opioid peptide receptor (KOPr) is a seven membrane receptor within the G-protein coupled receptor (GPCR) family. These are widely expressed throughout the central nervous system (CNS) (Mansour, Fox, Akil, \& Watson, 1995) and peripheral tissues (Feng et al., 2012; Wittert, Hope, \& Pyle, 1996). More specifically, the KOPr is one of four major opioid receptor families. The other three opioid receptor families are the MOPr, the delta opioid peptide receptor (DOPr), and the nociceptin receptor (Feng et al., 2012). These four opioid families have similar structures (Jordan et al., 2000) with most of the similarity seen in the seven transmembrane domains with the different families sharing about $60 \%$ amino acid identity (Burford, Traynor, \& Alt, 2015). The functionality of opioid receptors is greatly increased as they can form both homodimers with two identical monomers, and heterodimers: a dimer formed between two non-identical monomers (Durán-Prado, Malagón, Gracia-Navarro, \& Castaño, 2008). Heterodimers are more common in the opioid receptors with well known heterodimers formed between KOPr and DOPr subunits, and MOPr and DOPr subunits (Gupta, Décaillot, \& Devi, 2006). This heterodimerisation increases functionality of the receptors distinctively from homodimers of either subunit. It is thought that compounds binding specially to heterodimers are likely to have fewer side effects than their mono subunits (Gupta et al., 2006).

Activation of the KOPr subunit has been shown to mediate cardioprotection (Wang et al., 2010; Wu, Li, \& Wong, 1999) and increase heart rate without affecting blood pressure (Hajrasouliha et al., 2005). The MOPr subfamily has a role in regulating ionic homeostasis (legorova, Fisyunov, \& Krishtal, 2010), preconditioning against epileptic seizures (Rubaj, Gustaw, Zgodziński, Kleinrok, \& Sieklucka-Dziuba, 2000), and has a role in obesity by stimulating the intake of a high fat diet (Barnes, Holmes, Primeaux, York, \& Bray, 2006; Zuberi, Townsend, Patterson, Zheng, \& Berthoud, 2008). Neural differentiation has been shown to be promoted by DOPr activation (Narita et al., 2006), along with antidepressant and anxiolytic effects in rodents (Saitoh, Yoshikawa, Onodera, \& Kamei, 2005), and neuroprotection against hypoxic stress (Tian et al., 2008).

However, of all the effects the opioid systems have throughout the body, the main known effect and the main use of opioid receptor agonists as therapeutics are to do with pain mediation. Morphine is a well-known and widely used MOPr agonist for chronic pain 
(Jordan et al., 2000). It acts as a pain mediator by increasing the threshold for pain perception (Center, 2011). It is currently one of the most widely prescribed therapies for pain mediation due to its high potency as an analgesic. However, morphine also causes adverse effects including sedation, nausea, pruritus, and euphoria (Gupta et al., 2006). As excessive use of morphine and other known opiates such as heroin can lead to opioid addiction and tolerance within the nervous system (Feng et al., 2012), new compounds are being developed and tested for analgesic effects without causing dependence and other side effects. Compounds targeting the KOPrs have shown promise in this regard as attenuation of stimulant self-administration has been observed through the use of highly selective KOPr agonists (Prisinzano, Tidgewell, \& Harding, 2005)

\subsubsection{KOPr signalling pathways}

Activation of the KOPr system through agonist treatment stimulates various kinase pathways. As the KOPrs are from a class of GPCRs, part of their signalling pathways are to do with the G-protein subunits (Dogra \& Yadav, 2015). When an agonist binds to the receptor, the receptor changes shape causing the attached $\beta \gamma$ subunit to dissociate from Gai subunit. The now free $\beta \gamma$ subunit modulates calcium and potassium channels by stimulating potassium ion efflux and inhibiting calcium ion influx (Rusin, Giovannucci, Stuenkel, \& Moises, 1997). The Gai subunit inhibits adenyl cyclase activity leading to a decrease in cAMP (Taussig, Iñiguez-Lluhi, \& Gilman, 1993). Extracellular signal-regulated kinases 1 and 2 (ERK1/2) (McLennan et al., 2008) and c-Jun N-terminal kinase (JNK) (Kam, Chan, \& Wong, 2004) are both phosphorylated by the Gai subunit. This ERK1/2 phosphorylation through the Gai subunit is early phase phosphorylation occurring between 5 and $15 \mathrm{~min}$ after treatment. This phosphorylation is mediated through phosphoinositol 3-kinase (PI3K) and protein kinase C zeta (PKCद) (Belcheva et al., 2005). This part of the signalling pathway is known as the G-coupled cascade (figure 1.1). For a full review of KOPr signalling pathways, see Bruchas \& Chavkin, (2010).

When agonists bind to the $\mathrm{KOPr}$, the intracellular domain is also phosphorylated due to G protein receptor kinase 3 (GRK3) and $\beta$-arrestin recruitment (Dogra \& Yadav, 2015; Liu-Chen, 2004). This leads to KOPr internalisation and degradation (Li, Luo, Krupnick, Benovic, \& Liu-Chen, 1999), phosphorylation of p38 mitogen-activated protein kinase 
(p38 MAPK), and phosphorylation of ERK1/2. The latter is late phase phosphorylation occurring around $2 \mathrm{~h}$ after treatment (McLennan et al., 2008) and results in phosphorylation of cAMP response element binding protein (CREB) (Dogra \& Yadav, 2015). Transcription factors zif268 and c-Fos are induced as a result of p38 MAPK and CREB phosphorylation respectively (Bruchas \& Chavkin, 2010). This side of the signalling pathway is known as the $\beta$-arrestin recruitment pathway. 


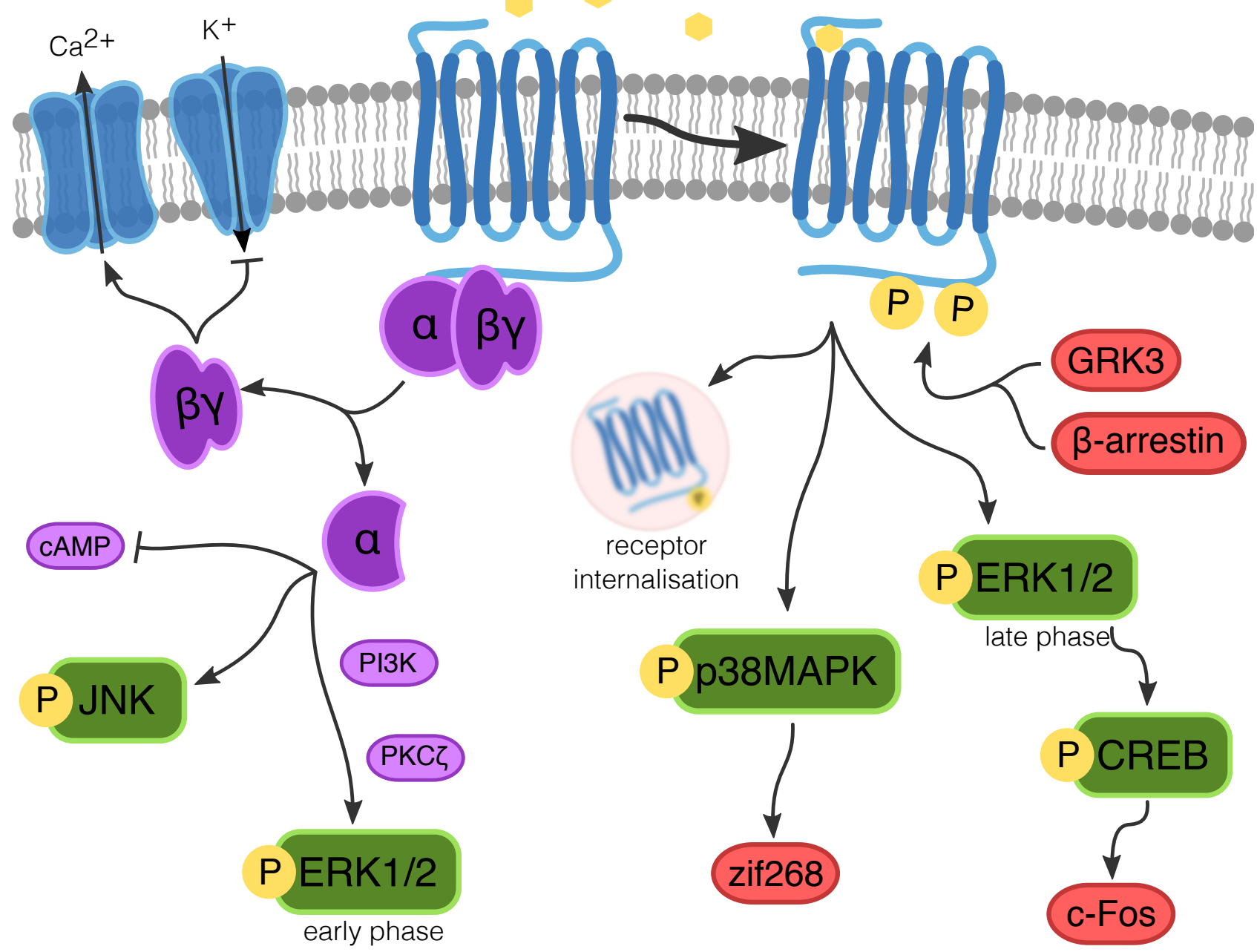

G-coupled cascade

$\beta$-arrestin pathway

Figure 1.1: KOPr signalling pathways

Binding of an agonist to the KOPr results in activation of two pathways: the G-coupled cascade and the $\beta$-arrestin pathway. The G-coupled cascade results in membrane hyperpolarisation, a decrease in CAMP through the adenyl cyclase pathway, and phosphorylation of JNK and early phase ERK $1 / 2$. The $\beta$-arrestin pathway results in receptor internalisation, and phosphorylation of p38 MAPK and late phase ERK1/2. Transcription factors such as zif268 and c-Fos are induced as a result.

Arrows indicate stimulation or activation. Blunt ended lines indicate inhibition. Adapted from Dogra et al. (2015).

CAMP $=$ cyclic adenosine monophosphate, CREB $=$ CAMP response element binding protein, ERK $1 / 2=$ extracellular signal-regulated kinases 1 and 2, GRK3 $=$ G-protein coupled receptor kinase $3, \mathrm{JNK}=c$-Jun $\mathrm{N}$-terminal kinase, $\mathrm{KOPr}=\mathrm{Kappa}$ opioid receptor, $\mathrm{p38} \mathrm{MAPK}=\mathrm{p} 38$ mitogen-activated protein kinase, $\mathrm{PI} 3 \mathrm{~K}=$ phosphoinositide 3-kinase, $\mathrm{PKC} \zeta=$ protein kinase $\mathrm{C} \zeta$ 


\subsubsection{Desirable effects of KOPr activation}

In addition to having analgesic effects, activation of the KOPr pathway can result in anti-addiction (Wang et al., 2010) and anti-pruritic effects (Ko \& Husbands, 2009). Both synthetic and endogenous KOPr agonists have been shown to reduce tolerance and addiction to morphine (Wang et al., 2010) as well as removing other adverse effects that morphine causes such as sedation, nausea, pruritus, urinary retention, euphoria, and a decreased ability to concentrate (Lahti, VonVoigtlander, \& Barsuhn, 1982). Activation of the KOPr system causes suppression of dopamine release from brain areas largely responsible for drug dependence (Wang et al., 2010), such as the mesolimbic pathway and the nigrostriatal pathway (Bruijnzeel, 2009). It can be suggested that drugs targeting the KOPr pathway will not cause dependence with use. This analgesic effect without physical dependence has been shown in both rats and mice (Lahti et al., 1982). Furthermore, the benefits of $\mathrm{KOPr}$ agonists can be extended from not causing dependence to treating dependence on other drugs of abuse. Cocaine is a drug well known for its ability to cause adaptations in brain chemistry and behaviour (Shippenberg, Zapata, \& Chefer, 2007) as well as addiction. Several studies involving animal models have shown a decrease in cocaine addiction after a KOPr agonist treatment (Schenk, Partridge, \& Shippenberg, 1999) indicating that KOPr agonists have potential as antiaddiction drugs (Wang et al., 2010) as well as non-addictive analgesics.

Nalfurafine is a KOPr agonist that is currently used for its anti-pruritic effects. In 2009, nalfurafine was implemented as an anti-pruritic agent for itch caused by haemodialysis in Japan in 2009 (Nagase, Kawamura, Kawai, \& Hayakawa, 2011). It was developed from the known KOPr antagonist naltrexone (Nakao \& Mochizuki, 2009) with early preclinical trials indicating no aversion or preference in rats tested with the conditioned place preference paradigm, nor did it show dependence in monkeys in a self-administration task (Nagase et al., 2011). After a phase 1 clinical trial indicated appropriate efficacy and safety when used to treat pruritus in haemodialysis patients (Nakao \& Mochizuki, 2009), it was implemented in Japan as a therapeutic agent (Nagase et al., 2011). Studies continued and a year long follow up trial indicated that nalfurafine has no difference in dependence liability compared to control treatment (Ueno, Mori, \& 
Yanagita, 2013) nor does it cause adverse effects on blood pressure, respiratory rate, body temperature, or pupil diameter. Nalfurafine therefore shows that KOPr agonists have strong potential as therapeutics for various conditions due to their variety of desirable effects.

A more recent promising effect found to be mediated by the KOPr system is remyelination. In multiple sclerosis, the myelin sheath produced by oligodendrocytes to insulate axons is destroyed (Goldenberg, 2012). Various methods for treatment of multiple sclerosis have been researched and all current drugs target the immune system (Du et al., 2016) and aim to reduce the symptoms. However no treatment for direct remyelination has been found (Borniger \& Hesp, 2016). The KOPr system has been suggested to play a role in the pathogenesis of multiple sclerosis. Through genetic deletion of $\mathrm{KOPr}$, symptoms of experimental autoimmune encephalomyelitis (EAE), the mouse model of multiple sclerosis, are increased whereas activation of the KOPr system decreases EAE symptoms (Du et al., 2016). Further studies corroborated this finding and showed that activation of the KOPr system with traditional agonist U50,488 promotes remyelination and aids in the maturation of oligodendrocytes in mice models (Mei et al., 2016). This finding once more demonstrates the potential of KOPr agonists as future therapeutics.

\subsubsection{Undesirable KOPr side effects}

Unfortunately KOPr activation can cause adverse effects such as dysphoria, psychometic effects (Crowley \& Kash, 2015), sedation, and diuresis (Wang et al., 2010) that must be considered in drug development. Studies in rhesus monkeys have shown KOPr agonists producing transient sedation and occasional vomiting (Mello \& Negus, 2000). Psychotomimetic, dysphoric, and aversive side effects have also been observed when KOPr agonists have been tested in humans (Pfeiffer, Brantl, Herz, \& Emrich, 1986).

These undesirable side effects are the main reasons that KOPr agonists aren't currently commonly used. For example, U50,488 was the first synthetic highly selective KOPr agonist to be created in 1982 (Simonin et al., 1998). Is it an analgesic that was shown to have potency about half that of morphine in mice antinociceptive assays (warm plate, hot plate, and tail flick) and showed a greater variability in the same tests in rats 
(Lahti et al., 1982). These desirable effects looked promising. However, further studies showed that U50,488 has adverse side effects including reduced locomotor activity or sedation, conditioned aversion, and an increase in salivation in mice (Simonin et al., 1998). These side effects make U50,488 an undesirable therapeutic although it still holds great importance as an experimental tool for the development of novel KOPr agonists.

\subsubsection{Bias agonism}

The desirable and undesirable effects caused by activation of the KOPr system occur due to the different signalling pathways. That is, the desirable analgesic effect is mediated through the G-coupled cascade whereas the side effects such as sedation and stress are mediated through the $\beta$-arrestin recruitment pathway (Dogra \& Yadav, 2015). More specifically, desirable effects are seen when phosphorylating early phase ERK1/2 (Simonson et al., 2015) whereas the undesirable side effects are seen more when phosphorylating late phase ERK1/2, p38 MAPK, and CREB (Bruchas et al., 2007). It is also now known and accepted that GPCRs have multiple conformations (Perez \& Karnik, 2005) and it is the ligand that determines which conformation is taken. Different conformations are responsible for the specific downstream effects (Urban et al., 2007). In terms of the KOPr system, it is possible to activate only the $\beta$-arrestin recruitment pathway or only the G-coupled cascade. This is a process known as biased agonism. Biased agonists are defined by the fact that they are able to modulate a receptor towards a biased receptor conformation without directly affecting the receptor activity on its own (Wisler, Xiao, Thomsen, \& Lefkowitz, 2014). It is therefore possible and highly sought after to find a KOPr agonist which is biased towards early phase ERK1/2 phosphorylation so that the beneficial analgesic effects are seen without the undesirable side effects.

\subsection{Learning and memory}

The mechanisms of learning and memory are among the most important mental processes that the brain controls. Memory is a broad term encompassing any behavioural changes brought about by experience, and the knowledge stored as a result of these experiences (Owen \& Brenner, 2012). The learning and memory process can be broken down into three stages: acquisition, consolidation, and retrieval. Acquisition is the 
learning phase. Consolidation and retrieval are what are often referred to as memory with consolidation being memory storage and retrieval being memory recall. All three stages are important aspects for memory and an impairment could occur at any stage.

\subsubsection{Memory classification}

One model of memory uses three main terms to describe different types of mammalian memory: working memory (WM), short term memory (STM), and long term memory (LTM) (Nadel \& Hardt, 2011). All memory systems are closely linked to each other with true definitions and explicit differences difficult to stipulate. However, STM is often defined by its duration - memories lasting from seconds to hours which are vulnerable to disruption including through head trauma and electroconvulsive shock (Bear, Connors, \& Paradiso, 2007). STM works on a biochemical level through altering the strength of relevant synapses in a process known as synaptic plasticity (Owen \& Brenner, 2012). Memories from STM can be consolidated into LTM through long term potentiation (LTP) if the increase in synapse strength becomes long lasting. The duration of LTM can range from days to years, and a set capacity of its storage limits has yet to be found (Nadel \& Hardt, 2011). Memories can also enter LTM directly from sensory input (Bear et al., 2007). Still within LTM, there are different types yet again. Cohen \& Squire (1980) separated LTM into procedural and declarative after studying what amnesiacs can and cannot form memories of. Here, procedural memory refers to "how" memory and declarative refers to "what" memory. That is, procedural memory refers to remembering motor skills such as riding a bike and does not require conscious thought (Tulving, 1986) whereas declarative memory is based around remembering facts. Procedural memory can further be divided into motor, perceptual, and cognitive memory (Mochizuki-Kawai, 2008) (figure 1.2). Declarative memory can also be divided further into episodic and semantic memory (Tulving, 1986). Semantic memory is defined as remembering information about the world, the meaning of words, and general knowledge. Episodic memory is based on remembering what has been experienced first-hand. All of declarative memory is conscious and requires active thought. 
WM can be though of as a particular type of STM as the duration is similar. However, WM is thought of more in terms of memory manipulation than merely its duration (Nadel \& Hardt, 2011). Information in WM exists in an active conscious state controlled by the central executive system. Another form of memory, reference memory, encodes information about a task that remains constant across trials, such as which arms in a radial arm maze always contain food (Nadel \& Hardt, 2011). This compares to WM which is constantly updated with which arms of the maze have already been visited. The current thesis will focus on recognition memory, a type of declarative memory. 


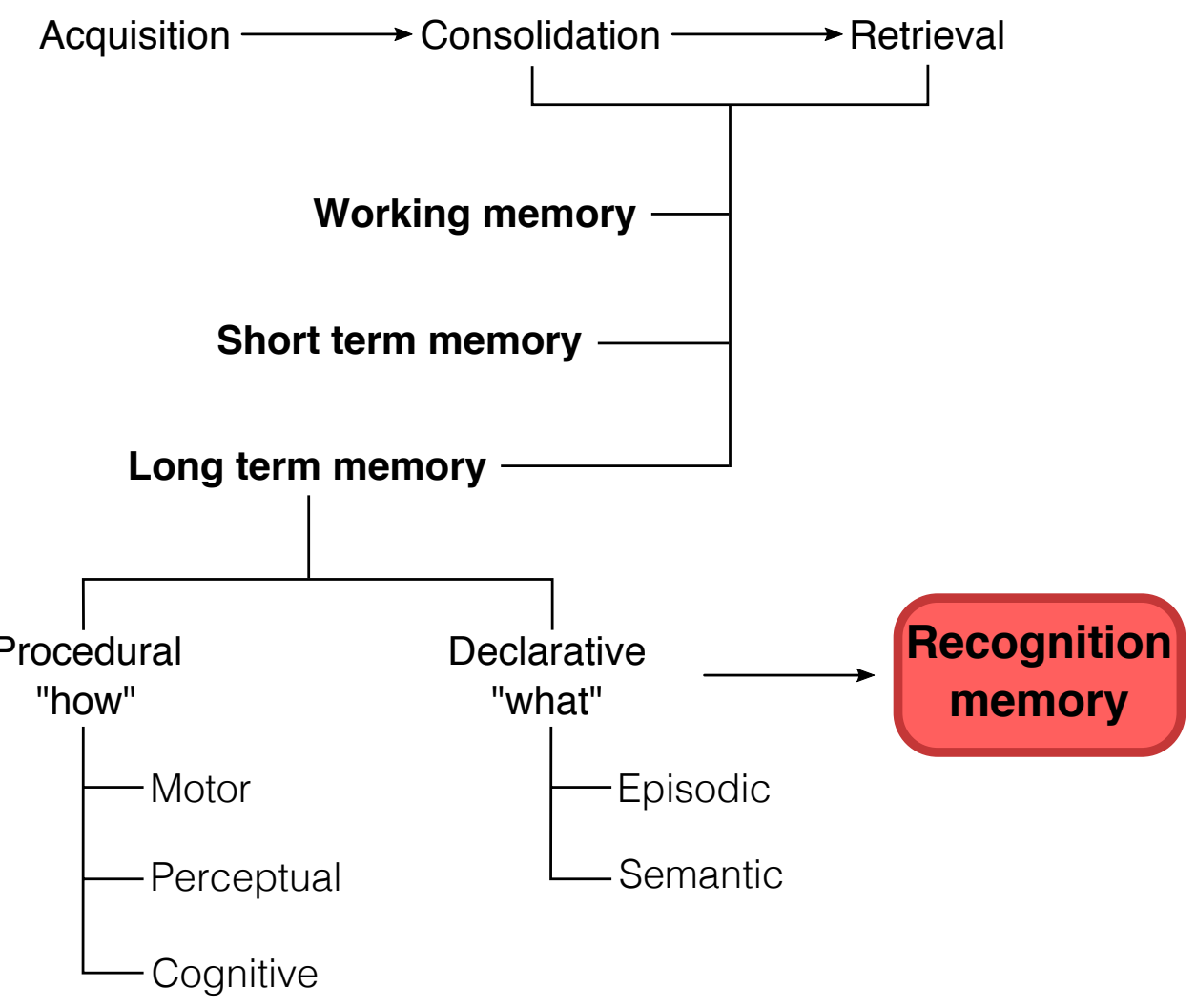

Figure 1.2: Learning and memory classification

Visual representation of the different types of memory and their subgroups. The current thesis focuses on recognition memory, a form of declarative long term memory. 


\subsubsection{Brain regions involved in recognition memory}

Memory is not held in one single area of the brain but rather is influenced through many different regions including the cerebral cortex, the cerebellum and basal ganglia, and the limbic system, specifically the hippocampus, thalamus, and amygdala. Not all regions are involved in all types of memory and connections have only been found between certain regions (Shu, Wu, Bao, \& Leonard, 2003). Specific parts of the cerebral cortex are important in different types of memory. The frontal and parietal lobes are largely involved in working memory (The European Dana Alliance for the Brain, 2003) with the frontal lobe also playing an important role in processing STM and retaining LTM (Fine, 2008). The temporal lobe is also known to play a role in forming LTM (Fine, 2008) and the PFC is a central structure in classical conditioning (Shu et al., 2003). The cerebellum, as a movement control centre (Bear et al., 2007), is very important in skill learning (under procedural memory) along with the basal ganglia (The European Dana Alliance for the Brain, 2003). Within the limbic system are brain regions many would argue as pivotal to learning and memory. Studies have shown that damaging the medial temporal lobe, specifically the hippocampal area, can cause both anterograde and retrograde amnesia as was the case with patient HM (Nadel \& Hardt, 2011). Since anterograde amnesia is most common with this type of brain injury, it is likely that the hippocampus is not the location for memory storage but rather that it plays a role in memory consolidation (Nadel \& Hardt, 2011). Furthermore, hippocampal lesions causing retrograde amnesia suggest that memory can depend on the hippocampus at one point, but then develop further and become independent later on. Nadal and Hardt (2011) also state that the thalamus plays an important role in working memory, alongside the PFC, the parietal cortex, and the cerebellum (see figure 1.3). The amygdala is involved in emotional responses (Bear et al., 2007) so it comes as no surprise to find that it is involved in emotional based learning and memory (Shu et al., 2003) such as fear conditioning.

The novel object recognition (NOR) task is used in rodents to look into declarative memory. More specifically, it is based around recognition memory (Antunes \& Biala, 2012). It was originally thought that brain regions involved in recognition memory included the hippocampus and amygdala after studies in monkeys showed recognition 
memory impairments after damage to the amygdala, the hippocampus, or both (Saunders, Murray, \& Mishkin, 1984; Zola-Morgan \& Squire, 1985). The level of impairment was shown to have a direct relationship with the amount of conjoint damage to the amygdala and hippocampus (Saunders et al., 1984). However, further studies focused on damaging more specific cortical regions in addition to lesions of the hippocampus, amygdala, or both (Murray \& Mishkin, 1986; Zola-Morgan, Squire, \& Amaral, 1989; Zola-Morgan, Squire, Clower, \& Rempel, 1993). Amongst these various studies, it was found that removing the rhinal cortex produces a recognition memory impairment equivalent to the impairment seen with a full hippocampal lesion (Murray \& Mishkin, 1986). Also, the perirhinal cortex was found to specifically play an important role in recognition memory independent of the hippocampus since memory impairments in object recognition behavioural tasks are seen when only the perirhinal cortex is damaged (For review see Winters, Saksida, \& Bussey, 2008). 

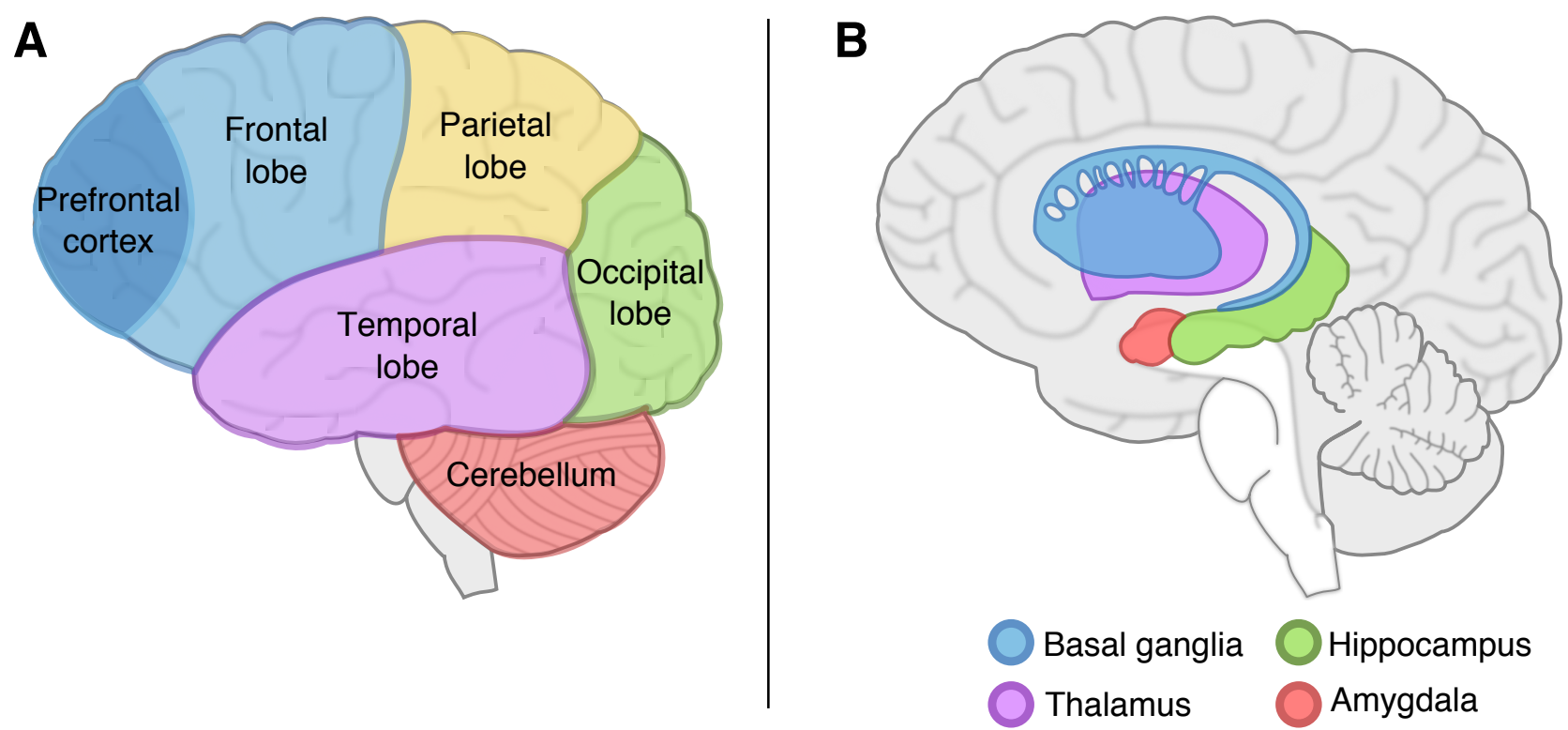

Figure 1.3: Brain regions involved in learning and memory

A The different lobes of the human brain. The PFC plays a role in classical conditioning. The frontal lobe is largely involved in WM, processing STM, and retaining LTM. The parietal lobe is also involved in WM. The temporal lobe has a role in forming LTM. The cerebellum is important in procedural memory.

B Areas of the midbrain involved in learning and memory. The basal ganglia is important in procedural memory. The thalamus has an important role in WM. The hippocampus plays a role in memory consolidation. The amygdala is involved in emotional learning such as fear conditioning. 


\subsubsection{Animal models of learning and memory}

Numerous behavioural tests have been developed and are carried out to investigate the effects different compounds have on learning and memory. Such tests developed for rats and mice include the passive avoidance task, the Morris water maze test, the radial arm maze, $T$ and $Y$ mazes, and the NOR task.

Firstly, the passive avoidance test. This is used to examine LTM through fear based stimuli (Gupta, Upmanyu, \& Vashist, 2012). Here, mice are trained through electric shock to stay on a wooden platform instead of stepping down to explore the test box: their natural tendency. A memory impairment would show a decreased latency in time to step down. That is, the animal would have failed to recall the electric shock and the fear response from the training phase.

A measure of spatial memory can be carried out using the Morris water maze (Gupta et al., 2012). A platform is submerged and hidden slightly below opaque water in an often circular maze and animals are trained where this platform is across multiple days. The location of the platform remains constant. If memory impairment occurs, the animal will take longer to find the platform. However, as the animal itself can initially be placed in different places of the circular tub, the test also looks into WM (Morris, 1984). Due to the stress induced from swimming, the pharmacokinetics of drugs tested may be slightly altered. This task looks into a mixture of working and reference memory.

Spatial WM can also be investigated using the radial arm maze (Gupta et al., 2012) or the $Y$ and $T$ mazes (Onaolapo, Onaolapo, Akanmu, \& Olayiwola, 2015). Here, an animal is placed in the centre of a maze with three or more 'arms' coming off it. The animal has an innate behaviour to spontaneously alternate which arm they enter so they enter each arm once before repeating any. An impairment in working memory would show as multiple or no entries into a singular arm.

The NOR task looks into working, but not reference, memory. The test works by comparing the amount of time an animal spends with a novel object over a familiar object (Braida, Donzelli, Martucci, Capurro, \& Sala, 2011) where the animal has a natural inclination to interact with the novel object more than the familiar. Normal memory will show more time spent with the novel object than the familiar object with a memory 
impairment showing approximately equal time with each object. Of course for this test to be valid the objects will have to be of similar interest to the animal in terms of size and manipulability.

\subsubsection{KOPr in learning and memory}

Dynorphins are a class of endogenous opioid peptides that bind specifically with KOPrs. Originally isolated from porcine pituitary (Goldstein \& Ghazarossian, 1980), there are now six known dynorphin peptides which vary in length. All are formed from the precursor prodynorphin (Knoll \& Carlezon, 2010). Dynorphins are found throughout the CNS but have greatest concentration in the anterior and posterior hypothalamus, the brainstem, the midbrain, the hippocampus, the striatum, the cortex, and the cerebellum (Goldstein \& Ghazarossian, 1980). Although dynorphins act primarily through the $\mathrm{KOPr}$ pathway, they have been shown to have slight affinities for the MOPr and DOPr pathways, as well as the non-opioid N-methyl-D-aspartic acid (NMDA)-type glutamate pathway (Schwarzer, 2009). The functions of dynorphins relate to learning and memory, stress response, and pain. It is thought that some types of epilepsy, addiction, depression, and schizophrenia have pathophysiological mechanisms involving dynorphins (Schwarzer, 2009).

Animal studies have shown that dynorphin causes both spatial memory impairment in rats through the Morris water maze test (Sandin et al., 1998), and a memory impairment reversal in rats when looking into acetylcholine release in the hippocampus (Hiramatsu, Mori, Murasawa, \& Kameyama, 1996). A possible explanation of these differences could be the differences between dynorphin itself and its primary metabolite. As it is, dynorphin activates the KOPr mediated mechanisms as an opioid and causes an improvement in impaired learning and memory. However, the opioid behaviours of dynorphin can be removed as it is rapidly degraded and the resulting dynorphin fragments can then produce non-opioid effects (Hiramatsu et al., 1996). It is these non-opioid fragments from the opioid parent molecule that may shed some light on the opposing effects dynorphin can have. However, Hiramatsu indicated that a non-opioid fragment of dynorphin, specifically des-tyrosine dynorphin, also improves memory impairment in mice models. 
Certain studies have shown a dose-dependent memory impairment through activation of the KOPr pathway by exogenous agonists. Sal A in the 8-arm radial maze (spatial memory) and the passive avoidance task (aversive memory) shows a memory impairment in spatial LTM, episodic, and aversive memory (Braida et al., 2011) and U50,488 at showed a memory impairment in a fear-conditioning paradigm and in the Morris water maze after a $2.5 \mathrm{nmol}$ intrahippocampal microinjection (Daumas et al., 2007). However, various other studies involving a passive avoidance test in rats and mice demonstrated that KOPr activation by U50,488, or novel agonists VA100 and VA101 reverses learning and memory impairment caused by scopolamine (Ghelardini et al., 2001; Hiramatsu \& Hoshino, 2004; Hiramatsu, Hoshino, Kameyama, \& Nabeshima, 2002; Hiramatsu, Murasawa, Nabeshima, \& Kameyama, 1998), carbachol (Hiramatsu, Hyodo, \& Kameyama, 1997; Hiramatsu \& Kameyama, 1998), and other amines (Ghelardini et al., 2001) in the passive avoidance test. These differences can be attributed slightly to the different memory tests used. That is when the memory impairment caused by $\mathrm{KOPr}$ agonists was seen, the tests used were behavioural using the step through passive avoidance task, the Morris water maze, or the NOR task. However, when the animals had first received a non-opioid treatment which induced a memory impairment, the $\mathrm{KOPr}$ agonists were shown to improve memory.

Furthermore, it has been suggested that KOPr activation is sufficient to impair memory as seen through the NOR task specifically (Paris, Reilley, \& McLaughlin, 2011). Using the peripherally restricted agonist, ffir- $\mathrm{NH}_{2}$ and $\mathrm{U} 50,488$ (0.3 mg/kg), $\mathrm{KOPr}$ activation impairs memory. This finding was corroborated in further NOR tasks using U50,488 in mice (Schindler, Li, \& Chavkin, 2010) and Sal A in rats (Braida et al., 2011). The conclusion of these KOPr agonists impairing learning and memory opposes the findings that KOPr activation by dynorphin improves it (Hiramatsu, Mizuno, \& Kanematsu, 2006). Thus, it is not possible to predict the effect a novel KOPr agonist will have on learning and memory and individual investigations must be carried out to identify the effect on learning and memory caused by each novel agonist. 


\subsubsection{Immediate early genes in learning and memory}

Immediate early genes (IEGs) are named after the fact that their transcription is activated rapidly and only lasts transiently after extracellular stimulation (Sheng \& Greenberg, 1990) by various means. Induction of IEGs within the nervous system can be brought about through seizure activity, stroke, sensory stimulation (whether it be noxious, non-noxious, olfactory, or visual), stress, and LTP and memory formation (Hughes \& Dragunow, 1995). Many IEGs encode transcription factors suggesting the products from IEG induction, often being late response genes (Sheng \& Greenberg, 1990), play a regulatory role. Such IEGs include zif/268, c-myc, the jun family, and the fos family. Members from the latter two families can from heterodimeric transcription factors which can alter the role of the IEG for example, a member of the fos family, c-Fos, acts as an activator of transcription factors when dimerised with c-Jun but when dimerised with JunB, it acts as a transcriptional repressor (Sheng \& Greenberg, 1990). One of the most commonly used IEGs in research is c-Fos as it has a low baseline expression level throughout the brain but is rapidly induced by various stimuli (Harlan \& Garcia, 1998) making it an appropriate tool to use in mapping activation of various brain regions in response to particular stimuli.

One way in which IEGs are induced in the CNS is during LTP and memory formation (Hughes \& Dragunow, 1995). Many experiments have been done which conclude that IEGs are rapidly induced in certain brain regions during learning and memory formation (see Tischmeyer \& Grimm, 1999 for a review). Specifically, IEG c-Fos has been seen in high amounts after presentation of novelty (Winters et al., 2008; Zhu, Brown, McCabe, \& Aggleton, 1995) which is an important aspect of the learning process. This finding of CFos being involved in learning has been strengthened through studies following repeated applications of the same stimulus. With this, c-Fos levels have decreased with repeated application of the one stimulus (Montag-Sallaz, Welzl, Kuhl, Montag, \& Schachner, 1999). If the c-Fos levels were determined by sensory stimulation itself, almost constant c-Fos levels would have been observed even after the stimulus was repeated. However, the decrease in c-Fos levels with repeated exposure show that c-Fos levels are determined by novelty, a memory-dependent process. 
It is highly possible that this induction of c-Fos is specifically induced by cAMP response element-binding protein (CREB) (Dogra \& Yadav, 2015) as studies with CREB inhibitors indicate an impairment in LTP and a disruption to c-Fos levels within the perirhinal cortex itself (Warburton et al., 2005; Warburton et al., 2003). The inhibition of CREB leading to a disruption in c-Fos levels can be explained as CREB is a transcription factor which binds to CAMP response element (CRE) which in turn mediates c-Fos induction centrally (Tischmeyer \& Grimm, 1999). This also makes c-Fos a good target to look into for KOPr agonists as late phase ERK phosphorylation after activation of $\mathrm{KOPr}$ causes CREB phosphorylation (Dogra \& Yadav, 2015) (see figure 1.1).

\subsubsection{CREB in learning and memory}

CREB is a stimulus induced transcription factor that mediates various biological functions. The phosphorylation of CREB can be induced by stimuli including peptide hormones, growth factors, and neuronal activity (Shaywitz \& Greenberg, 1999). This activation occurs as a response to an increase in cellular concentration of the calcium ion or cAMP (Silva, Kogan, Frankland, \& Kida, 1998). The phosphorylation of CREB can control various biological functions such as cell proliferation and differentiation, adaptive responses, and adaptations to drugs of abuse (Shaywitz \& Greenberg, 1999). CREB also plays an important role in LTM formation (Yin \& Tully, 1996).

Experiments in Aplysia (Dash, Hochner, \& Kandel, 1990), Drosophila (Tully, 1991), and mice (Bourtchuladze et al., 1994; Phillips \& LeDoux, 1992; Strupp \& Levitsky, 1984) have all come to the conclusion that CREB is important in LTM formation with agents that disrupt CREB activity resulting in a blockage of LTM. However, the specific isoforms of CREB that are induced could have a say in whether LTM is enhanced or impaired. CREB isoforms are either activators or repressors (Silva et al., 1998) and the effect of CREB on LTM depends on the ratio of these two isoforms (Yin \& Tully, 1996). Different isoforms are a result of alternative splicing, alternative start sites for gene transcription, or alternative promoters being used for transcription (Silva et al., 1998). If there is a greater concentration of activator isoforms than repressor isoforms, LTM is facilitated and enhanced. However, if there is a greater concentration of repressor isoforms than 
activator isoforms, the formation and retrieval of LTM is prevented (Yin \& Tully, 1996) resulting in a memory impairment.

\subsection{Salvinorin $A$ and novel analogues}

Salvinorin A (Sal A), the main active ingredient from the naturally psychoactive plant Salvia divinorim (Braida et al., 2011), is structurally different from most known KOPr agonists, such as $\cup 50,488$, as it is a non-nitrogenous, non alkaloid diterpene (Roth et al., 2002) (see figure 1.4). Sal A studies in humans see reporting of visual distortions, feelings of unreality, and depersonalisation (Siebert, 1994). In murine models, Sal A showed antinociceptive effects in the tail-flick, hotplate, and abdominal constriction assays (John, French, \& Erlichman, 2006; McCurdy, Sufka, Smith, Warnick, \& Nieto, 2006). These effects had a short duration of action which agree with previous studies in non-human primates (Schmidt et al., 2005). Sal A has also been shown to reduce anxiety and depression in both mice and rat models (Braida et al., 2009). However, due to the high hallucinogenic effects of Sal $A$ in humans and its poor pharmacokinetic properties, it is unlikely to be developed into a therapeutic. Analogues of Sal A however, may prove likely therapeutics for anti-addiction.

The 2-methoxy methyl analogue of Sal A (MOM Sal B) has been shown to significantly attenuate cocaine seeking behaviours in rats whilst suppressing sucrose reinforcements (Morani, Ewald, Prevatt-Smith, Prisinzano, \& Kivell, 2013). Another analogue of Sal A, $\beta$-tetrahydropyran Salvinorin B ( $\beta$-THP), has shown potent analgesic and antiinflammatory effects in mice using the tail withdrawal assay, formalin-induced inflammatory pain model, and a paclitaxel-induced neuropathic pain model (Paton et al., 2017). However, neither of these two analogues are likely to be extended into therapeutics in humans due to adverse effects including sedation and depressive effects (Morani et al., 2013; Simonson et al., 2015). Sal A still proves a rich experimental tool for demonstrating KOPr activation and other novel analogues may still be found as therapeutically beneficial.

Mesyl Sal B, a derivative of Sal A with a mesylate group at the C2 position, shows a longer duration of action compared to parent molecule Sal A indicating an improvement in metabolic stability and bioavailability (Simonson et al., 2015). Anti-cocaine and anti- 
addiction effects are also shown in mice with the administration of Mesyl Sal B at $0.3 \mathrm{mg} /$ $\mathrm{kg}$ (Simonson et al., 2015). Ethy Sal A, a C16 analogue of Sal A, is a potent KOPr agonist which has been shown to reduce drug-seeking behaviour as it successfully attenuated drug seeking behaviour in a cocaine-prime-induced reinstatement model in rats at 0.3 $\mathrm{mg} / \mathrm{kg}$ (Riley et al., 2014) without causing sedation, an undesirable side effect. Substitution of a bromine onto the $\mathrm{C} 16$ position of Sal A produces Bromo Sal A, another novel analogue with high potency and selectivity for the KOPr (see table 1.1). Bromo Sal A has also been tested for reducing drug-seeking behaviour in rats (Riley et al., 2014). Here, the number of presses on the previously active level was significantly reduced suggesting it attenuates cocaine-prime-induced drug-seeking behaviour. As locomotor data indicated the Bromo Sal A did not cause sedation in the rats, Bromo Sal A also shows promise as a possible future therapeutic. These three novel analogues: Ethy Sal A, Mesyl Sal B, and Bromo Sal A, will be tested in this thesis due to their promise in previously reported data. 


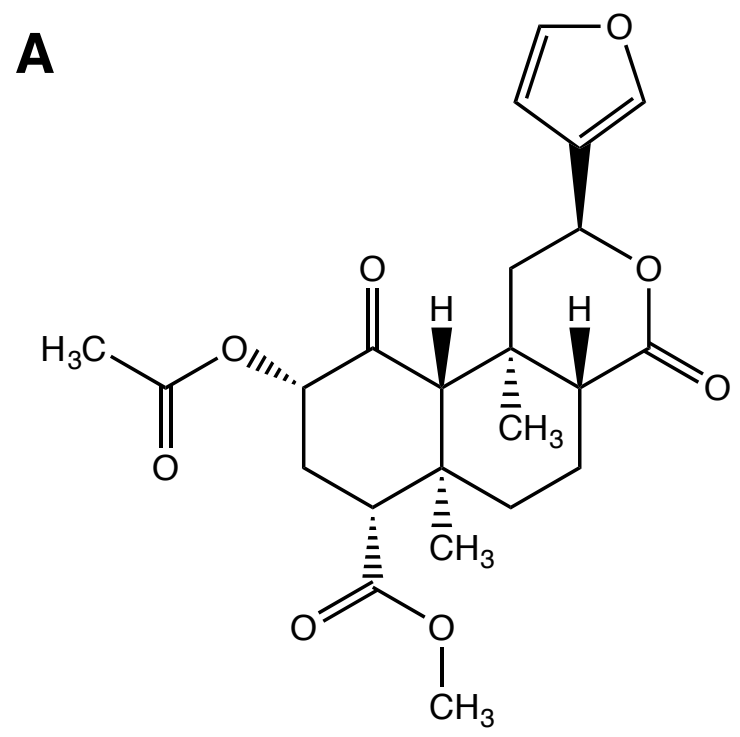

B<smiles>CN(C(=O)Cc1ccc(Cl)c(Cl)c1)[C@@H]1CCCC[C@H]1N1CCCC1</smiles>

Figure 1.4: Structures of Sal A and U50,488

Chemical structures of KOPr agonist Sal A (A) and prototypical KOPr agonist U50,488 (B). Sal A differs structurally from prototypical KOPr agonists as it is a non-nitrogenous diterpene. 


\section{U50,488}

Salvinorin A

Ethynyl

Salvinorin A

Mesyl

Salvinorin B

Bromo

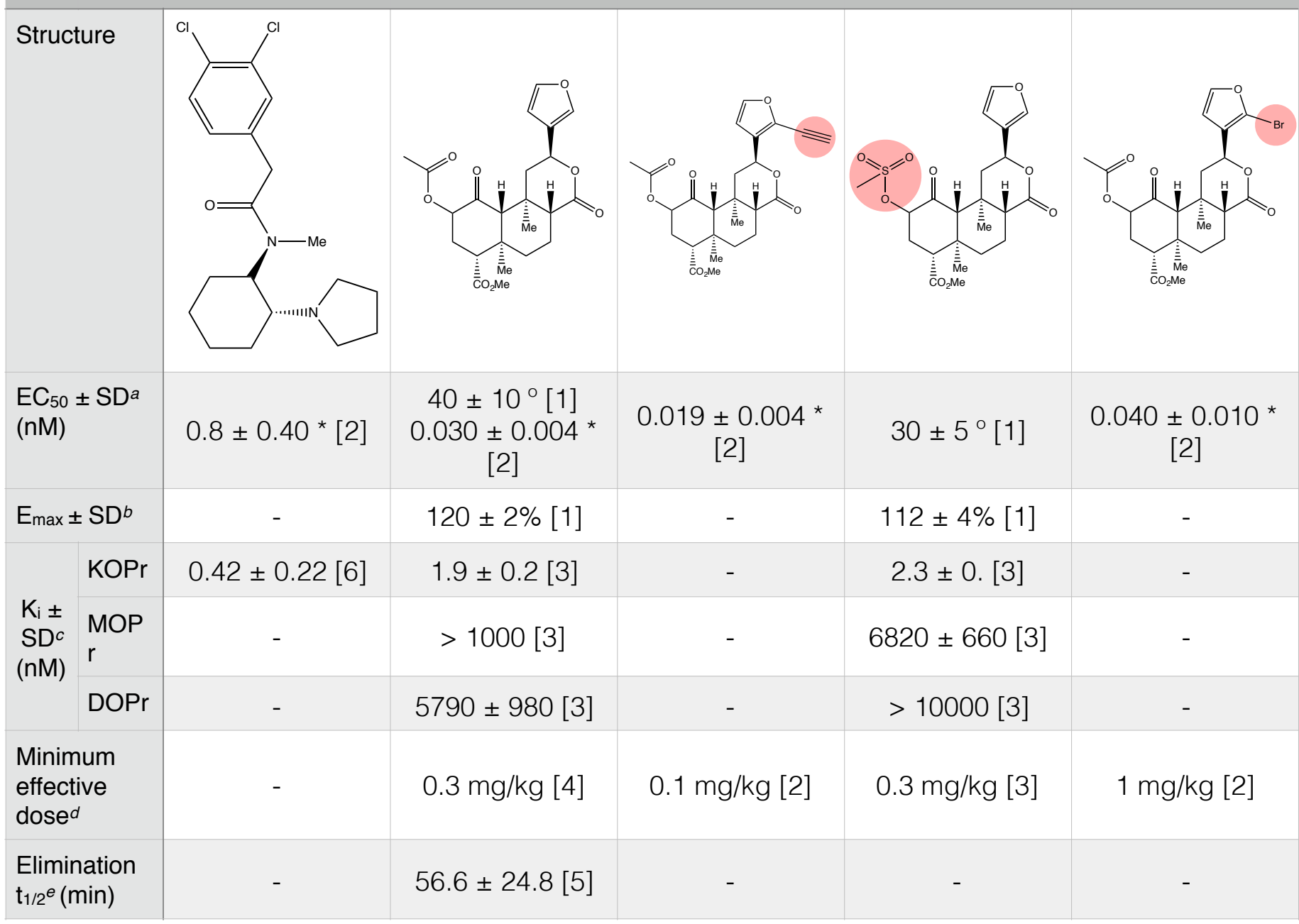

Table 1.1: Pharmacokinetic properties of U50,488, Sal A, and novel analogues

Prototypical KOPr agonist U50,488, and C2 and C16 analogues of Sal A and their pharmacokinetic properties. Structural changes compared with Sal A are indicated in red circles.

[1]: Harding et al. (2005), [2]: Riley et al. (2014), [3]: Simonson et al. (2015), [4]: Morani et al. (2013), [5]: Schmidt et al. (2005), [6]: Beguin et al. (2008)

${ }^{a} \mathrm{EC}_{50}=$ effective concentration required to reach $50 \%$ of the maximum possible response measured using the ${ }^{0}\left[{ }^{35 S}\right]$ GTP $\gamma S$ assay or through *testing for inhibition of forskolin-induced cAMP accumulation in human $\mathrm{KOPr}$ expressing $\mathrm{CHO}$ cells. ${ }^{b} \mathrm{E}_{\max }=$ the percentage stimulation of binding compared with prototypical KOPr agonist, U50,488 (500 nM). 'Binding affinities of Sal A and novel analogues at the $\mathrm{KOPr}, \mathrm{MOPr}$, and DOPr using [125I]IOXY as a radioligand. aMinimum effective dose required to have a significant effect on cocaine reinstatement. eElimination half time measured in Rhesus monkeys 


\section{Aims and hypotheses}

The aim of this thesis was to evaluate the effects of KOPr agonists on learning and memory. In order to evaluate this effect, a suitable protocol for the NOR task in rats firstly needed to be established. Two plausible NOR protocols were chosen with the aim of finding a protocol where known a KOPr agonist, Sal A or U50,488, is found to produce an impairment in short or long term recognition memory. After establishing a suitable protocol with a positive control, Sal A analogues were tested. It was hypothesised that the novel analogues would cause little or no impairment in memory.

It is known that observation of novelty activates the perirhinal cortex. My second aim was therefore to use immunohistochemistry $(\mathrm{IHC})$ to evaluate levels of the IEG, c-Fos, between vehicle and positive control treated animals. As rats who don't experience a memory impairment identify only the novel object as novel, it was expected for there to be a small amount of c-Fos activation after vehicle treatment. Rats who were treated with the positive control and experienced a memory impairment would identify both the novel and the familiar object as novel meaning it was expected for memory impaired rats to have a higher level of c-Fos activation within the perirhinal cortex. 


\section{Methods}

\subsection{Animals and housing}

Male Sprague-Dawley rats (Rattus norvegicus) weighing 280 to $410 \mathrm{~g}$ were housed in the animal facility of the School of Biological Sciences, Victoria University of Wellington. Animals were housed $2-4$ per cage in a temperature $\left(20{ }^{\circ} \mathrm{C}\right)$ and humidity $(50 \%)$ controlled room. Lighting was on a controlled 12:12 hour light:dark cycle with the light period beginning at 0700 hours. Animals had free access to food and water (Diet 86, Sharpes Stock Feed) except during testing. All testing was carried out during the light period. Prior to any experimentation, animals were handled for at least two days to minimise stress. In accordance with the Arrive guidelines: replacement, reduction, refinement, rats had previously been used in other behavioural experiments (eg conditioned place preference, forced swim test, light/dark test) with a minimum rest period of seven days between tests. Any naive rats used were reused in other tests unless they were sacrificed for the current experiment. All experiments were approved by the Victoria University of Wellington Animal Ethics Committee.

\subsection{Drugs and treatment}

Rats were injected with U50,488 (10 mg/kg) (Sigma-Aldrich, St. Louis, MO, USA), Salvinorin A (0.3 or $1 \mathrm{mg} / \mathrm{kg}$ ), Mesyl Sal B (0.3 or $1 \mathrm{mg} / \mathrm{kg}$ ), Ethy Sal A (0.3 or $1 \mathrm{mg} / \mathrm{kg}$ ), Bromo Sal A (1 mg/kg) (courtesy of Prof. T. Prisinzano, University of Kansas, Lawrence, $\mathrm{KS}, \mathrm{USA}$ ), or norBNI (10 mg/kg) (Sigma-Aldrich). All drugs were dissolved in a 2:1:7 mixture of DMSO:Tween-80:Milli-Q water and administered at a volume of $1 \mathrm{~mL} / \mathrm{kg}$. Control animals were injected with the 2:1:7 vehicle. All agonist treatments, except norBNI, were administered via an intraperitoneal (ip) injection. norBNI was administered via a subcutaneous (sc) injection.

Pretreatment times differed between treatments depending on their rate of uptake. Sal A was administered 5 min prior to testing, both Bromo Sal A and Ethy Sal A were administered 10 min prior to testing. Mesyl Sal B had a pretreatment time of 45 min and 
U50,488 and the vehicle were administered $15 \mathrm{~min}$ prior to testing. The antagonist, norBNI, was administered 24 h before a U50,488 treatment on test day.

\subsection{Behavioural assays}

\subsubsection{NOR task methods development}

We investigated various methods of the NOR task in order to evaluate which protocol is most sensitive and suitable to evaluate the effects of KOPr agonists on learning and memory. The first protocol utilised a three-phase design following the methods of Carey el al. (2009). Briefly, rats were habituated to the test box on day 1 by placing the rats in the empty box for $5 \mathrm{~min}$. On day 2, test day, the experiment was broken down into three 10 min phases with a 10 min intertrial interval (ITI) between each phase. In phase I, two identical objects were secured in place on either side of the test box. In phase II, one of the objects was moved into the corner of the test box. In phase III, the previously moved object was replaced with a novel object. A diagram outlining the experimental protocol is shown in figure 3.1A. Three trials following this method were carried out with subtle changes between each trial to optimise the protocol. A total of 23 rats were used across these trials.

The second protocol used a two-phase protocol following the methods of Braida et al (2011). Phase I, the familiarisation phase, involved placing the rat in the test box with two identical objects stuck down on either side of the box. In phase II, the test phase, one of these objects was replaced with the novel object (figure 3.1B). Here, two trials were attempted with a total of 22 rats used. The trials were identical as the results showed promise at first.

In both protocols, the objects were wiped down with a cloth saturated with natural rat odour by storing it in dirty housing, between each phase and trial. At the end of the day, the objects were cleaned with Virkon detergent (DuPont, Suffolk, UK) and stored in the housing previously used by rats of similar ages. The test box was also wiped down with Virkon to remove any scent trails left between phases and trials.

In all phases, the time that any part of the rat, excluding the tail, spends in contact with each object and the time that the nose of the rat is within $1 \mathrm{~cm}$ of the object facing 
and engaged with it (sniffing, manipulating, etc) was recorded using Smart software. From these times, a recognition index $(R I)$ was calculated as $(N /(F+N)) \times 100$ where $N$ is the time spent with the novel object and $F$ is the time spent interacting with the familiar object. Times spent with each object were recorded in seconds. Rats were excluded from data analysis if they had a RI of 0 or 100 , that is, if they only interacted with one of the objects.

All experiments were carried out using ambient fluorescence lighting (20 - 60 Lux) in the presence of white noise to mask background noises.

\subsubsection{Final NOR test procedure}

In order to asses the affect of KOPr agonists on learning and memory, a protocol based on that of Schindler et al. (2010) was used. This protocol differed from earlier ones trialled mainly in the aim to reduce stress experienced by the rats. Also, both STM and LTM were considered at first in order to investigate which was most sensitive for KOPr agonists.

Rats were handled on days 1 - 2 before being subject to three days of habitation to the empty test box for 30 min each day. Rats were also handled on habituation days bringing them to a total of five handling days. On day 6 , rats were familiarised to the constant object by securing two identical objects on either side of the test box. To ensure that the rats were definitely familiar with this object, they were placed in the text box with the familiar objects three times, for a period of 6 min each, with 10 min between each time as done previously (Schindler et al., 2010). On this day, the rats also received a mock ip injection of saline $(0.9 \% \mathrm{w} / \mathrm{v}, 1 \mathrm{~mL} / \mathrm{kg})$ to minimise stress experienced from the injection. For testing STM, the test phase came 90 min after completion of the familiarisation phase. When testing LTM, the test phase came $24 \mathrm{~h}$ after the familiarisation phase, on day 7. Here, rats received an ip injection of treatment before being placed in the test box for 6 min with one familiar object and one novel object (see figure 3.2C). The time each rat spent exploring the different objects were recorded using Smart software. The RI was calculated from these times as $N /(F+N)) \times 100$ where $N$ is the time spent with the novel object and $F$ is the time spent with the familiar object. All times were recorded in seconds. 
Rats were excluded from data analysis if they had a RI of 0 or 100 , that is if they only interacted with one of the objects. When comparing STM and LTM, a total of seven trials were carried out to increase the number of subjects used to 30 for STM (15 vehicle, 15 U50,488 (10 mg/kg)) and 39 for LTM (19 vehicle, 20 U50,488 (10 mg/kg)).

After finalising the protocol to a suitable standard to test the novel KOPr analogues, a within subjects designed was implemented such that each rat received all treatments over a nine week period. Treatments used were vehicle, U50,488 (10 mg/kg), Sal A (0.3 $\mathrm{mg} / \mathrm{kg}$ and $1 \mathrm{mg} / \mathrm{kg})$, Ethy Sal A (0.3 mg/kg and $1 \mathrm{mg} / \mathrm{kg})$, Mesyl Sal B $(0.3 \mathrm{mg} / \mathrm{kg}$ and 1 $\mathrm{mg} / \mathrm{kg}$ ), and Bromo Sal A (1 mg/kg). To ensure there wasn't any bias introduced by different treatments interacting with each other, treatments were given a week apart to ensure full metabolism of the previous treatment. A balanced latin square design was also implemented so that each group of three rats received the nine treatments in a different order (table 3.1). If a rat excluded based on the criteria mentioned above for one treatment, the excluded treatment was administered again after completion of the original latin square. If a rat excluded from two or more treatments, they were removed from the cohort and their data was not included in analysis. This is due to needing a complete data set for each animal for the statistical analysis to be valid. The original cohort consisted of 24 male rats with 2 that excluded, resulting in an experimental cohort of 22 rats for all treatments.

To determine whether the effect of the novel KOPr agonists were KOPr mediated, a group of 6 rats received the selective KOPr antagonist, nor-BNI, (10 mg/kg, sc) $24 \mathrm{~h}$ before treatment with the positive control U50,488. The animals used for this group were selected based on their size after the nine week latin square design, and their RI obtained from previous treatment with the positive control, U50,488. Rats with a low RI were selected as they showed a recognition memory impairment. 

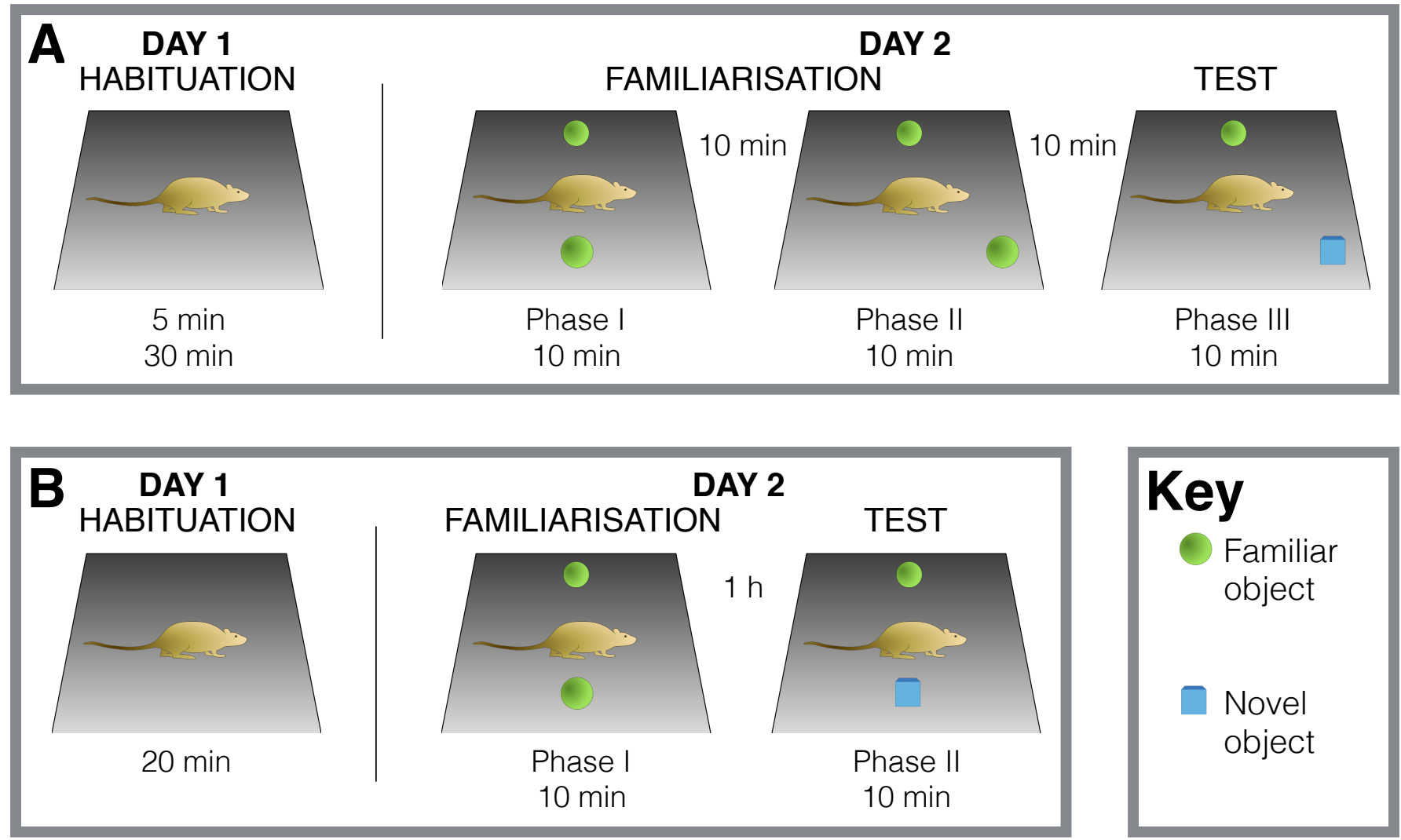

Key

Familiar object

Novel object

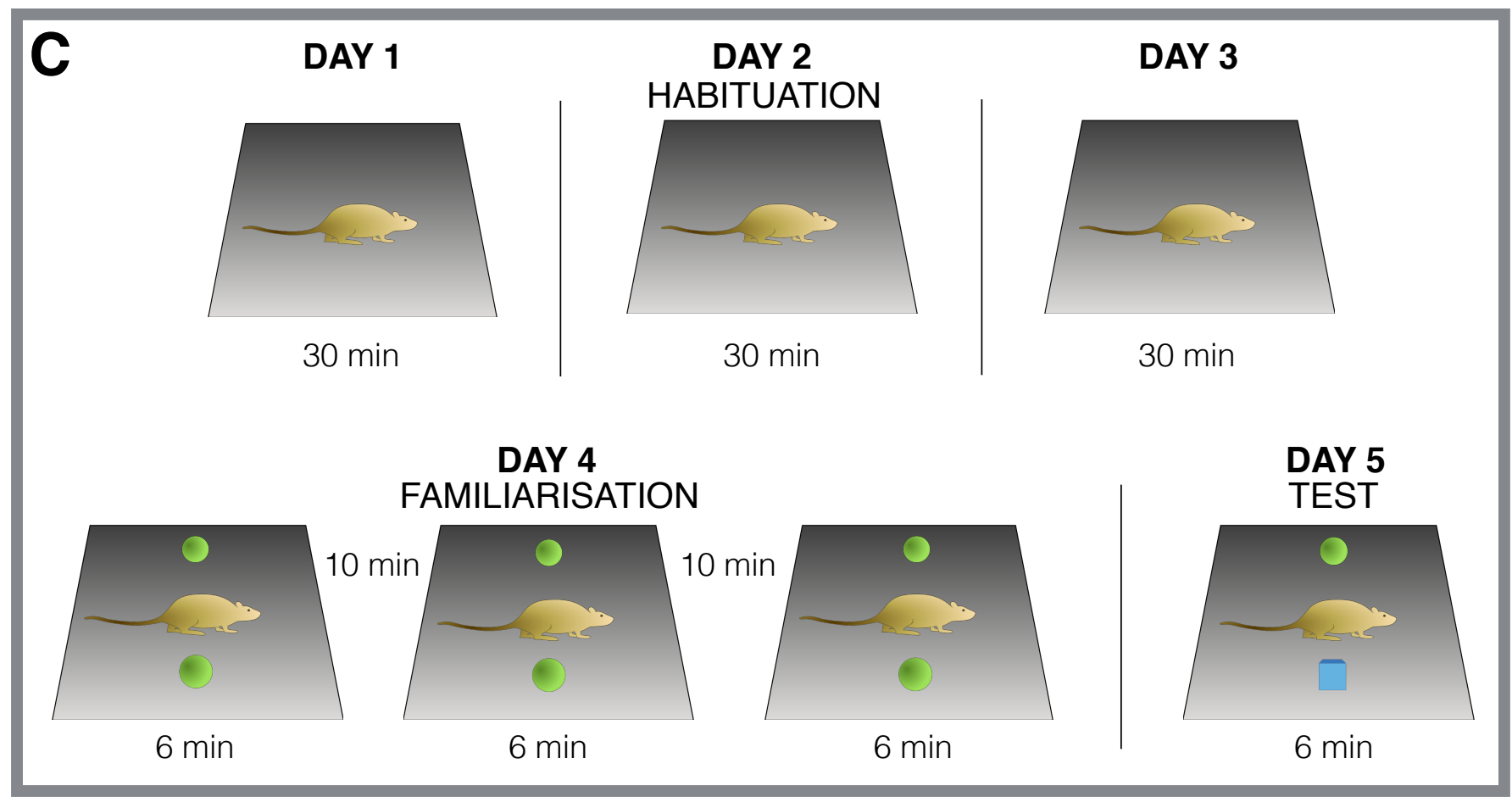

Figure 3.1: Visual representation of NOR methods trialled

A Three-phase design following the methods of Carey et al. (2009). Rats were habituated to the test box for 5 or $30 \mathrm{~min}$ on day 1. Two familiarisation phases and one test phase (10 min each) occurred on day 2. B Two-phase design following the methods of Braida et al. (2011). Rats were habituated to the test box for 20 min on day 1 . Familiarisation and test phases happened on day 2 with both phases lasting $10 \mathrm{~min}$. C The final protocol used following methods of Schindler et al. (2010). Rats were habituated to the test box for 30 min a day for three days (day 1 - 3). Familiarisation phase was day 4 and was broken into three stages of 6 min each with 10 min between each stage. Day 5 had the test phase which was 6 min in duration. 


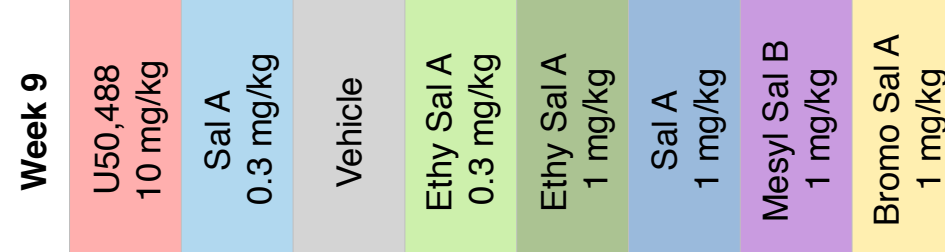

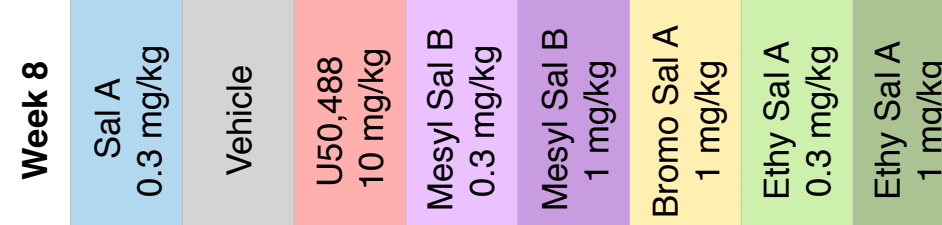

ก1

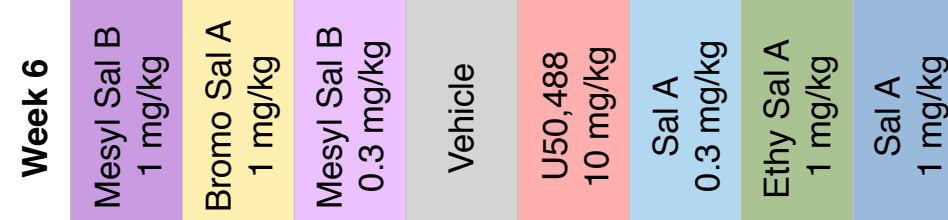

ำำ

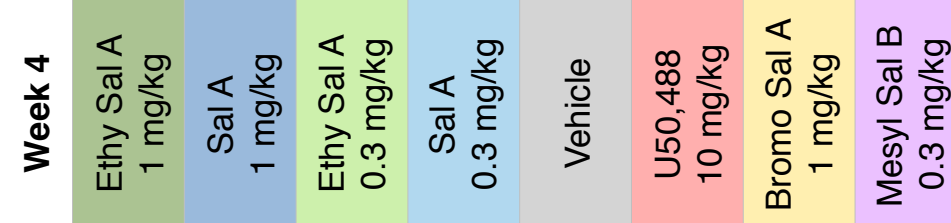

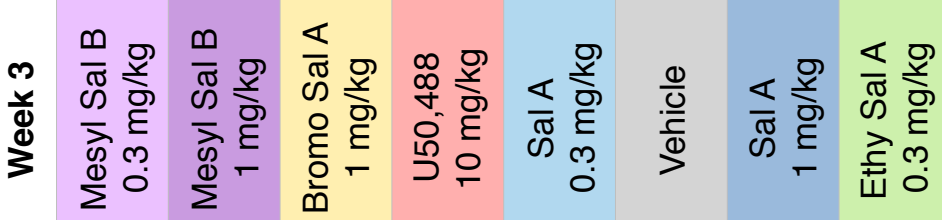

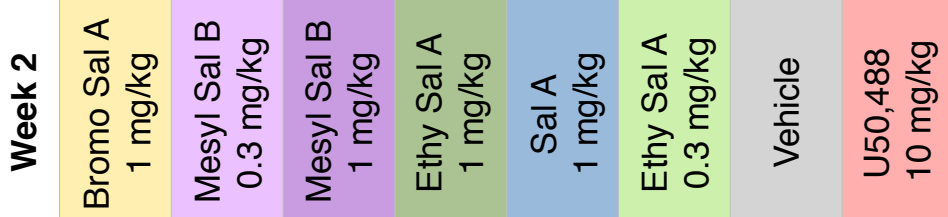

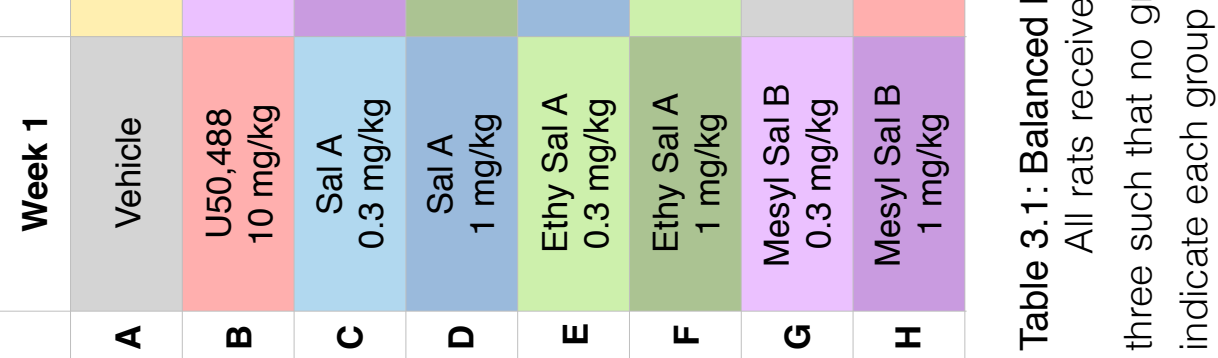

¿

욘

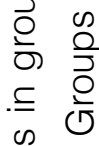

진

+ 잉

(ब)

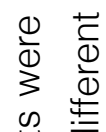

艺

है

Ф)

+

竞

它

Ф)

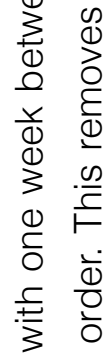

을

$\stackrel{2}{\rightleftharpoons}$

$\stackrel{\infty}{\subseteq}$.

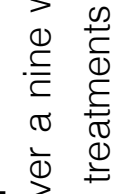

든

क 足

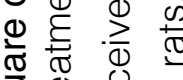

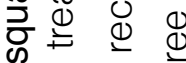

㕃 윽

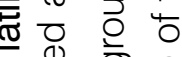




\subsubsection{Forced swim test for immunohistochemistry methods development}

As using immunohistochemistry (IHC) to investigate the level of c-Fos activation in specific brain regions was new to our laboratory, the protocol used first had to be optimised. In order to carry out this optimisation, a c-Fos inducing treatment was needed. Subjecting a rat to acute stress is known to induce c-Fos in various parts of the brain. Cullinan et al. (1995) looked into the effect of both swim stress and restraint stress on c-Fos expression in the rat brain. It was found that a $10 \mathrm{~min}$ forced swim test (FST) in a 36 ${ }^{\circ} \mathrm{C}$ water tank produced high c-Fos expression $30 \mathrm{~min}$ after stress onset. This c-Fos increase was seen most dramatically in the infralimbic cortex, the cingulate cortex, the paraventricular hypothalamic nucleus, and area CA1 of the hippocampus. Looking into c-Fos expression after acute swim stress is a common method used when investigating the effects of antidepressants on stress related brain areas (Bellchambers, Chieng, Keay, \& Christie, 1998; Cullinan et al., 1995; Duncan, Johnson, \& Breese, 1993; Duncan, Knapp, Johnson, \& Breese, 1996; Muigg et al., 2007).

For a positive control needed to optimise the $\mathrm{IHC}$ protocol and antibodies, rats were subjected to $10 \mathrm{~min}$ of forced swimming without any habituation or handling to the room or chamber. $30 \mathrm{~min}$ after stress onset, the rats were euthanised with $\mathrm{CO}_{2}$ and perfused (see appendix 2) with phosphate buffered saline (PBS) $(0.14 \mathrm{mM} \mathrm{NaCl}, 2.68$ $\mathrm{mM} \mathrm{KCl}, 8.1 \mathrm{mM} \mathrm{Na}_{2} \mathrm{HPO}_{4}, 1.47 \mathrm{mM} \mathrm{KH}_{2} \mathrm{PO}_{4}$; appendix 1) followed by paraformaldehyde in PBS (pH4, 4\%) (PFA). Brains were extracted and postfixed in PFA on ice for $2 \mathrm{~h}$. They were then cryoprotected by being transferred to a $30 \%$ sucrose in PBS solution at $4{ }^{\circ} \mathrm{C}$ overnight or until they reached equilibrium with the sucrose solution and sunk to the bottom of the tube. Brains were frozen and stored at $-80{ }^{\circ} \mathrm{C}$ until needed for sectioning.

The main two areas that were used to identify c-Fos are the infralimbic cortex and the cingulate cortex (Cullinan et al., 1995). Thus, only the section of the brain between 10 and $13 \mathrm{~mm}$ (interaural measurements) were used for sectioning (figure 3.2). This region was separated from the rest of the brain using a brain block. The brains were then sliced into $20 \mu \mathrm{m}$ coronal sections on a cryostat with every fourth section being mounted onto super frost slides for staining. 

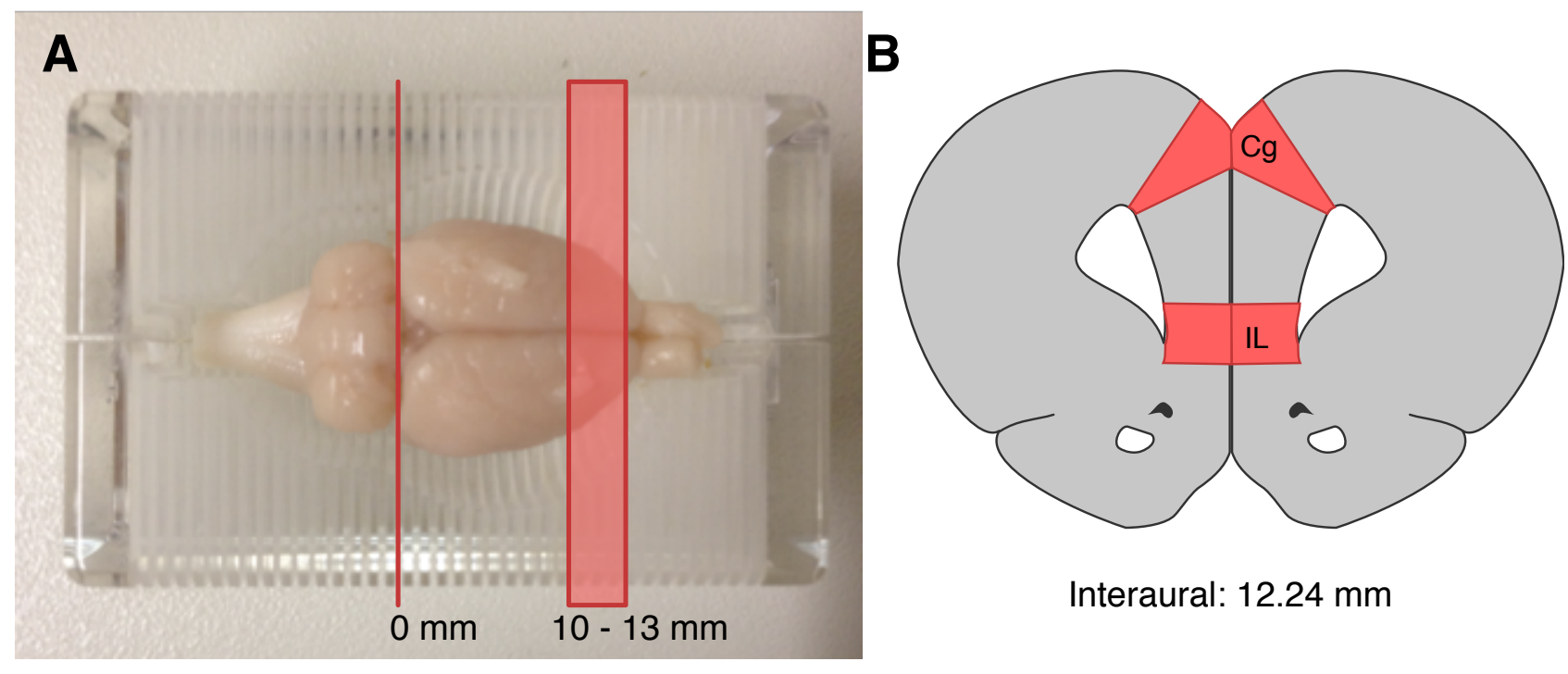

Interaural: $12.24 \mathrm{~mm}$

Figure 3.2: Rat brain regions used in forced swim stress

A Brain block indicating interaural measurements. The section from $10-13 \mathrm{~mm}$ was sectioned to look into the cingulate and infra limbic cortices. B Rat brain section at interaural measurement $12.24 \mathrm{~mm}$ indicating the two brain regions of interest. $\mathrm{Cg}=$ cingulate cortex, $\mathrm{IL}=$ infralimbic cortex. 


\subsubsection{Immunohistochemistry}

Mounted brain sections were washed twice with PBS followed by a further wash in PBS with Triton X-100 (0.1M, 0.2\%) (PBS-T) to permeabilise the cells. All washes were for 5 min. Slides were incubated in $1.5 \% \mathrm{H}_{2} \mathrm{O}_{2}$ in PBS for 15 min to quench endogenous peroxidase activity. Slides were then washed in twice in PBS and once in PBS-T followed by a 30 min incubation in blocking buffer consisting of goat serum in PBS (Ultra-Sensitive ABC Peroxidase Rabbit IgG Staining Kit, Thermo Fisher Scientific) to prevent non-specific staining. Sections were then incubated in the primary anti-c-Fos antibody (1:2000, abcam, ab 190289) at $4{ }^{\circ} \mathrm{C}$ for $24 \mathrm{~h}$ followed by a further two washes in PBS and one wash in PBS-T. The secondary biotinylated goat anti-rabbit antibody (Ultra-Sensitive ABC Peroxidase Rabbit IgG Staining Kit, Thermo Fisher Scientific) was added to the sections for $2 \mathrm{~h}$ at room temperature (1:200) before a further two washes in PBS and one wash in PBS-T. Sections were then incubated in an avidin-biotin peroxidase complex (UltraSensitive ABC Peroxidase Rabbit IgG Staining Kit, Thermo Fisher Scientific) for $1 \mathrm{~h}$ at room temperature under constant rotation, followed again by two washes in PBS and one in sodium acetate $(0.1 \mathrm{M}, \mathrm{pH} 6)$. Commercial nickel-cobalt intensified diaminobenzidine (DAB) kit (Thermo Fisher Scientific, Leicestershire, UK) was then added for approximately $90 \mathrm{~s}$ to visualise the DAB. Slides were washed two more times in PBS before being dehydrated through a series of graded ethanol (5 min washes in 70\%, 80\%, 95\%, 95\%, $100 \%, 100 \%$ ), cleared with xylene and coverspilled with DPX mounting media (Thermo Fisher Scientific).

\subsubsection{Quantification of c-Fos positive cells}

The number of c-Fos positive cells within the perirhinal cortex was quantified using stereology and the fractionator method. This technique was employed in order to accurately estimate the number of c-Fos positive cells within the perirhinal cortex without introducing bias. Specific aspects of stereology that were implemented are based around sampling in a random systematic manner (Schmitz \& Hof, 2005). For the purposes of the current experiment, every fourth section was taken within the interaural measurements 4.08 to $5.76 \mathrm{~mm}$ starting from a random section determined using a random number 
generator. A brain block was used to isolate the intramural section $0-6 \mathrm{~mm}$. Sectioning began at the $6 \mathrm{~mm}$ (figure 3.3A) end with sections from $5.76 \mathrm{~mm}$ being mounted onto slides. Each section was $20 \mu \mathrm{m}$ thick with the height of the disector or counting frame being $16 \mu \mathrm{m}$ to account for the edge effect: a phenomenon that can result in incorrect counting of cells due to the image being cut off before sufficient information of the particle in question can be seen. Since the particles in question here were neuron cell bodies which are round in shape, only a small outer edge is required to reduce this effect.

The fractionator method was employed in counting the number of c-Fos positive cells. Here, a grid measuring $200 \times 200 \mu \mathrm{m}$ was placed randomly on top of the perirhinal cortex. In each square of the grid, the c-Fos positive cells were counted within a $50 \times 50$ $\mu \mathrm{m}$ counting frame (based on literature (West, Slomianka, \& Gundersen, 1991). See figure 3.3B) due to cell density in the perirhinal cortex. Neurons were counted as c-Fos positive if they were within the counting frame in focus. Cells touching either the bottom or left sides of the frame were not counted (Figure 7D). The brain region of interest: the perirhinal cortex, was identified by comparison of the stained section with Paxinos and Watson's Brain Atlas (Figure 7C).

After finding the total number of cells in each counting frame used across the grid, the volume fraction was used to estimate the total number of c-Fos immunoreactive cells in the brain region of interest. This is the fractionator method which independent of tissue deformation (Kristiansen \& Nyengaard, 2012) and is therefore beneficial for frozen, imperfect sections. Here, the number of neurons in the subregion $(N)$ is estimated based on the following equation

$$
N=\sum Q \frac{t}{h} \frac{1}{\operatorname{asf}} \frac{1}{s s f}
$$

where $Q$ is the total number of neurons counted in the counting frames, $t$ is the thickness of the sections, $\mathrm{h}$ is the height of the disector, the areal sample fraction (asf) is a ratio of the area of the counting frame to the area of the grid $(a(f r a m e) / a(x, y$ step $))$, and the section sampling fraction (ssf) is defined as the fraction of the sections that have been sampled (West et al., 1991). 

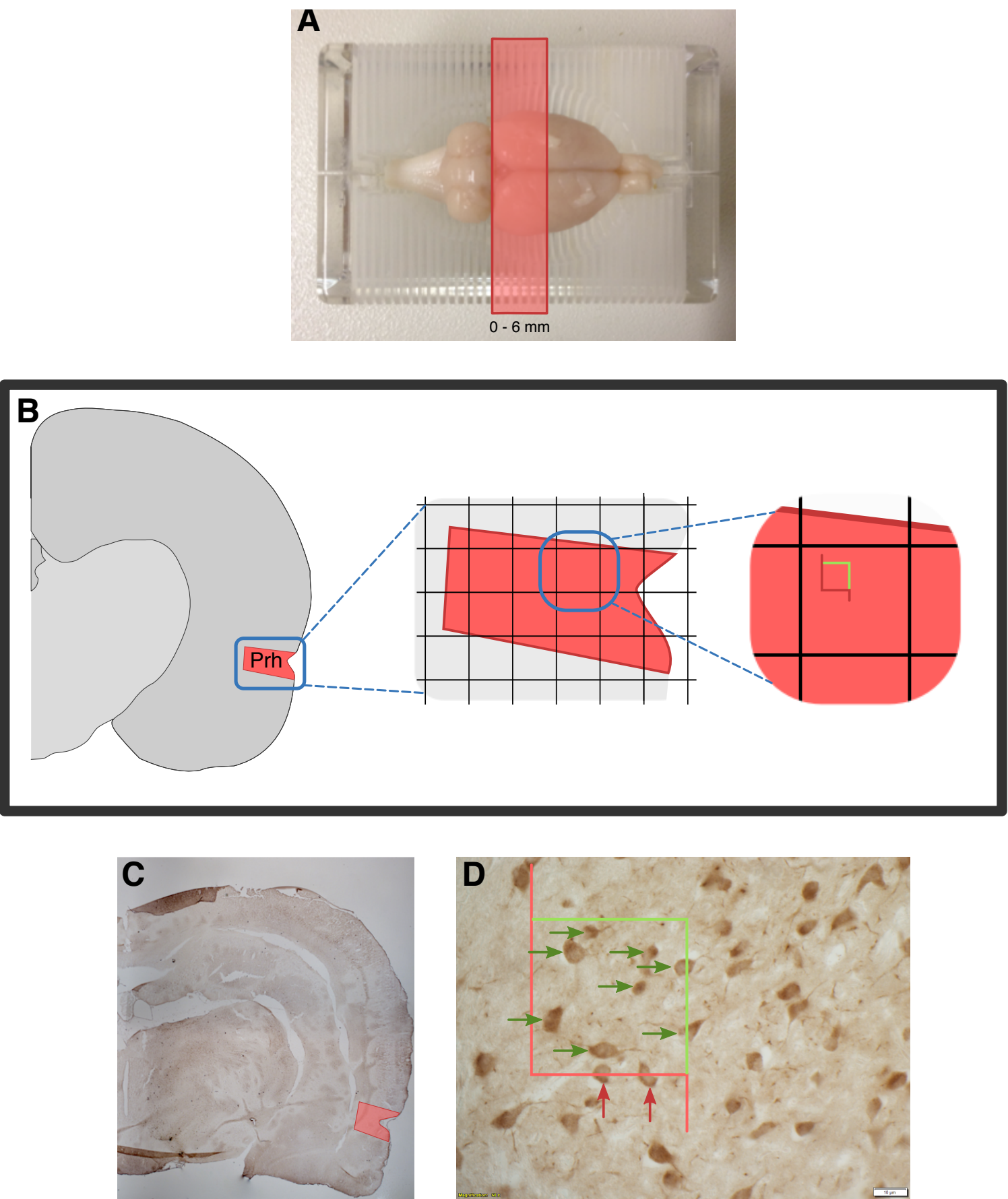

Figure 3.3: Stereology method used when quantifying c-Fos positive cells

A Brain block indicating section of rat brain taken for quantifying c-Fos positive cells. Sectioning started from the $6 \mathrm{~mm}$ end. Sections between 4.08 and $5.76 \mathrm{~mm}$ were collected.

Schematic view of the process taking when quantifying c-Fos cells. The brain region of interest, the perirhinal cortex, was identified through comparison with the brain atlas. A $200 \times 200 \mu \mathrm{m}$ grid was placed randomly over the region of interest. Within each square on the grid, a $50 \times 50 \mu \mathrm{m}$ counting grid was systematically placed. c-Fos positive cells within the counting frame were counted. C Photo of a section indicating the position of the perirhinal cortex (2x magnitication). D Neurons touching the left or bottom of the counting frame are not included in the c-Fos count (red arrows). Neurons touching the top and right sides are quantified (green arrows) (60x magnification).

Prh $=$ perirhinal cortex . 


\subsection{Materials}

The test box used in the NOR behavioural assays was a light grey open field box (45 $\times 45 \times 35 \mathrm{~cm}$ ) with a black mat on the floor. The mat was used to increase contrast between the box and the animal in order for the video tracking software (SMART Video Tracking Software) to successfully track the animal. Objects when optimising the protocol were marbles and dice. In the first trial run of the protocol, smaller objects were used with the familiar object being a clear marble $(1.5 \mathrm{~cm}$ diameter), and the novel object being a green die $(1.5 \times 1.5 \times 1.5 \mathrm{~cm})$. In all other trials, larger objects were used with the familiar object being a clear marble $(3 \mathrm{~cm}$ diameter) and the novel object being a coloured square $(3 \times 3 \times 3 \mathrm{~cm}$, eight smaller dice stuck together).

Various 3D printed objects of approximately $3 \times 3 \times 3 \mathrm{~cm}$ in size were used for the final protocol. These were either grey or yellow in colour such that the familiar and novel object in one test week were opposite in colour. To save on cost of printing, novel objects from one week were further used as the familiar object the following week. Novel objects were always new to the animals on test day. Grey objects were pyramids, spheres, arrows, dodecahedra, and hexagons. Yellow ones were cylinders, donuts, cones, and vases (see figure 3.4). For the behavioural assay before $\mathrm{HC}$, the familiar object was a grey dodecahedron and the novel was a yellow cone.

When optimising the $\mathrm{IHC}$ protocol, rats were subject to a forced swim test to induce stress which is known to induce c-Fos in certain brain regions. This forced swim test was carried out in a clear cylinder $40 \mathrm{~cm}$ tall with a $19 \mathrm{~cm}$ diameter. This was filled with $36{ }^{\circ} \mathrm{C}$ tap water to a height of $30 \mathrm{~cm}$. 


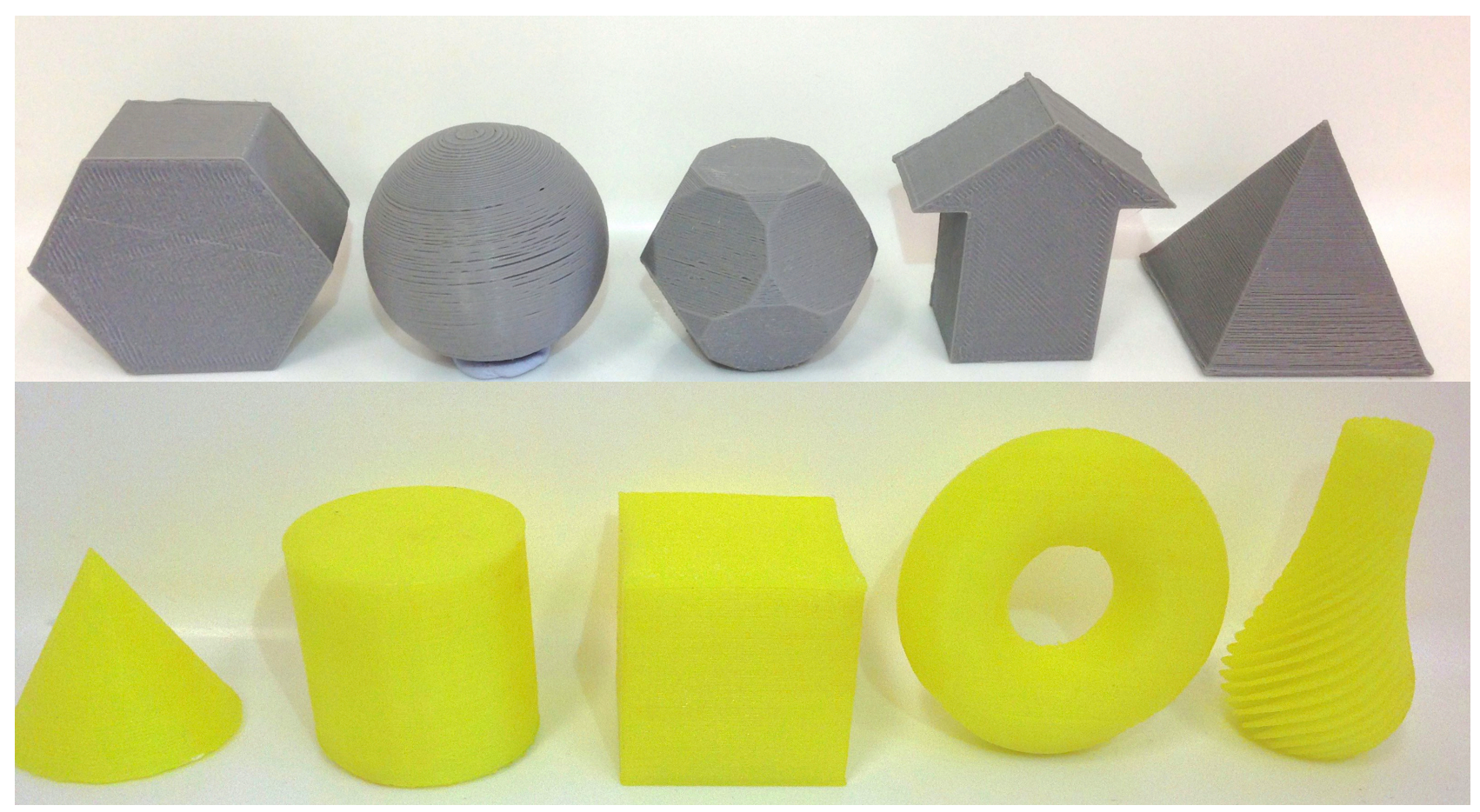

Figure 3.4: Objects used throughout the NOR tests

Grey objects used were (from left to right) a hexagon, a sphere, a dodecahedron, an arrow, and a pyramid. Yellow objects used were a cone, a cylinder, a cube, a donut, and a vase. 


\subsection{Statistical analysis}

All statistical tests were performed using GraphPad software (Prism 6, GraphPad Software Inc., La Jolla, CA, USA). During methods development for the NOR task, an unpaired Student's t-test was used to compare the difference between two treatments. When multiple treatments were analysed from the same trial, an one-way analysis of variance (ANOVA) was used. When the ANOVA produced a significant result, that is when $p<0.05$, Dunnett's multiple comparison post-hoc test identified where the significant differences were. For the latin square design based NOR protocol and the norBNI reversible tests, results were analysed using repeated measures one-way ANOVA with Dunnett's multiple comparisons. When quantifying c-Fos counts, Spearman's rank-order correlation was used as the results did not meet Gaussian distribution. All data was tested for normality with a Shapiro-Wilk test. When normality was not met, a Mann-Whitney test was used in place of Student's t-test and the Friedman test was used in place of repeated measures one-way ANOVA. All values presented are the mean \pm SEM and statistical significance was determined when $p \leq 0.05$. 


\section{Results}

\subsection{Novel object recognition}

\subsubsection{Larger objects allow for greater object exploration time}

As this experiment was new to our laboratory, several optimisation experiments were performed. Initially, a three-phase protocol, with a 5 min habituation period, and small objects (green die: $1.5 \mathrm{~cm}$ cube, clear marble: $1.5 \mathrm{~cm}$ diameter) were used. Using this design, the average time rats spent with either object was $12.5 \pm 2.2 \mathrm{~s}$ (mean \pm SEM) across both trials and over all three-phases. Sal $A$ at $1 \mathrm{mg} / \mathrm{kg}$ showed an average $\mathrm{Rl}$ of $78.1 \pm 6.6(n=5)$, with vehicle having a $R$ I of $68.3 \pm 13.3(n=4)$ (figure 4.1A). The difference between the two treatments showed no significance $(p=0.4794)$. As this protocol followed that set out previously by Carey et al. (2009) who showed a significance difference between the Sal A and vehicle, these insignificant results were unexpected.

Due to the short amount of time each rat spent interacting with either object, the dice and marbles were replaced with larger objects (clear marble: $3 \mathrm{~cm}$ diameter, coloured dice: $3 \mathrm{~cm}$ cube) in attempt to increase object interaction time. Using this protocol, the average time spent with any object was increased to $18.2 \pm 3.2 \mathrm{~s}$. However, the $\mathrm{Rl}$ scores still showed no significant difference (figure 4.1B) between Sal A (86.5 \pm 4.0, $n=5)$, and vehicle $(78.4 \pm 8.9, n=4, p=0.3935)$. Due to the larger object size increasing the interaction time with each object, the larger objects was used for all following experimental tests.

\subsubsection{Increasing habituation time increases object exploration}

In order to maximise time spent interacting with the objects, a longer habituation period was trialled. Previously, a longer familiarisation time to the test box has been shown to increase the time interacting with objects and decrease the latency to first object contact (Wilkinson, Herrman, Palmatier, \& Bevins, 2006). Thus habituation time was increased from a single 5 min period to a total of $30 \mathrm{~min}$, made up of three $10 \mathrm{~min}$ habituation periods separated by 10 min intervals outside the test area, to reduce interference from environmental exploration on object interaction. 
The increase in habituation time resulted in an increase in object interaction time from $18.2 \pm 3.2 \mathrm{~s}$ to $20.5 \pm 2.7 \mathrm{~s}$. Unfortunately significance was still not seen between treatment and vehicle, with Sal A showing a RI of $87.1 \pm 8.6(n=3)$ and vehicle treated rats having a $\mathrm{RI}$ of $87.1 \pm 5.9(n=3, p=0.8174)$ (figure $4.1 \mathrm{C})$. Although the increase in habituation time did not produce a significance difference as expected from previous experiments (Braida et al., 2011), there was an increase in object exploration time so use of a 30 min habituation time matching the timing of the experiment was used in following experimental tests.

\subsubsection{Using a two-phase protocol increases the sensitivity of the positive control}

In all the previous trials, there were three-phases to each run. Each phase took 10 min with a 10 min ITI totalling 50 min from the start of phase I. Since Sal A has a short half life (see table 1.1), this extended test could mean that Sal A could be completely metabolised before or during phase II. Due to this possibility, we adopted a protocol used by Braida et al. (2011). That is, Sal A will be trialled in a protocol with only two 10 min phases will be used with a $1 \mathrm{~h} \mathrm{ITI}$.

Utilising this two-phase protocol, the effects of Sal A (0.3 and $1 \mathrm{mg} / \mathrm{kg}$ ) in 2:1:7 DMSO:Tween 80:Milli-Q water vehicle on memory impairment was evaluated. The vehicle trials showed a RI of $75.8 \pm 6.0(n=6)$, Sal A at $0.3 \mathrm{mg} / \mathrm{kg}$ had a RI of $75.5 \pm 9.5(n=5)$, and Sal $A$ at $1 \mathrm{mg} / \mathrm{kg}$ had a RI of $77.5 \pm 3.3(n=10)$. Once more, no significant difference was seen between any of the groups ( $p=0.9587$, one-way ANOVA) (figure 4.1D). 
A

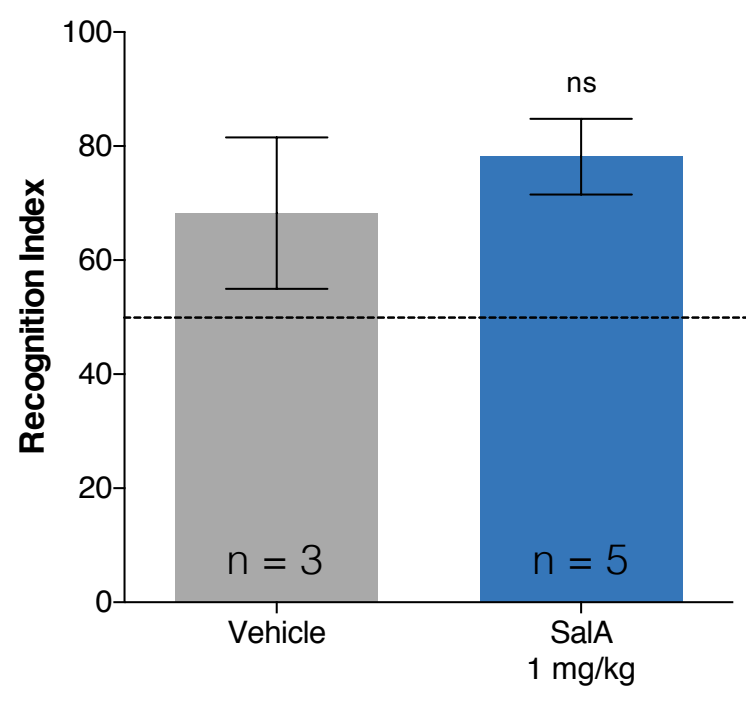

Treatment

C

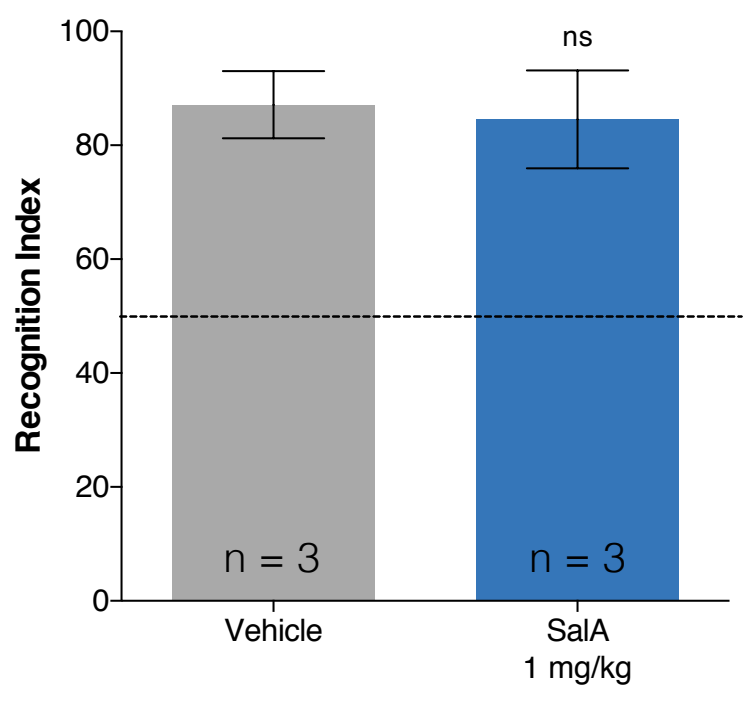

Treatment
B

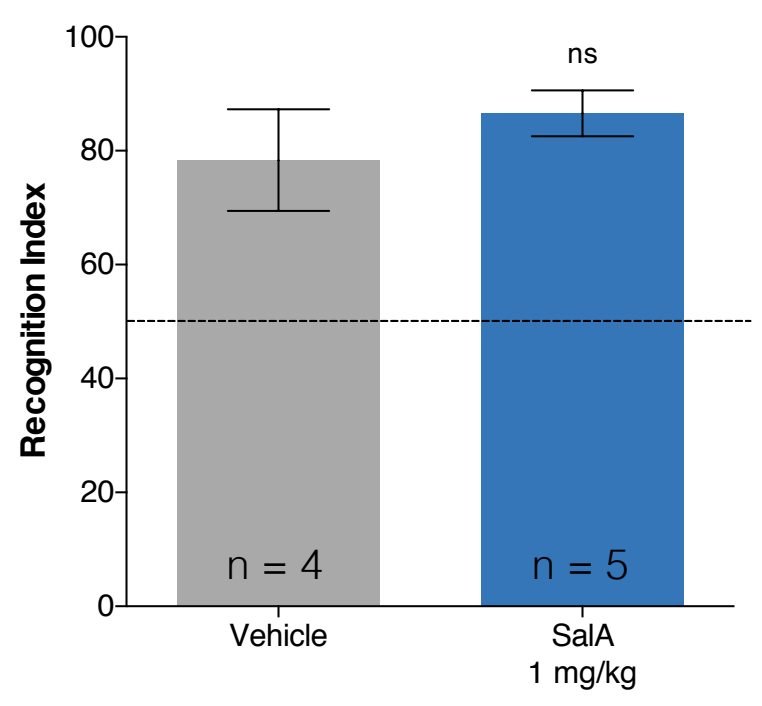

Treatment

D

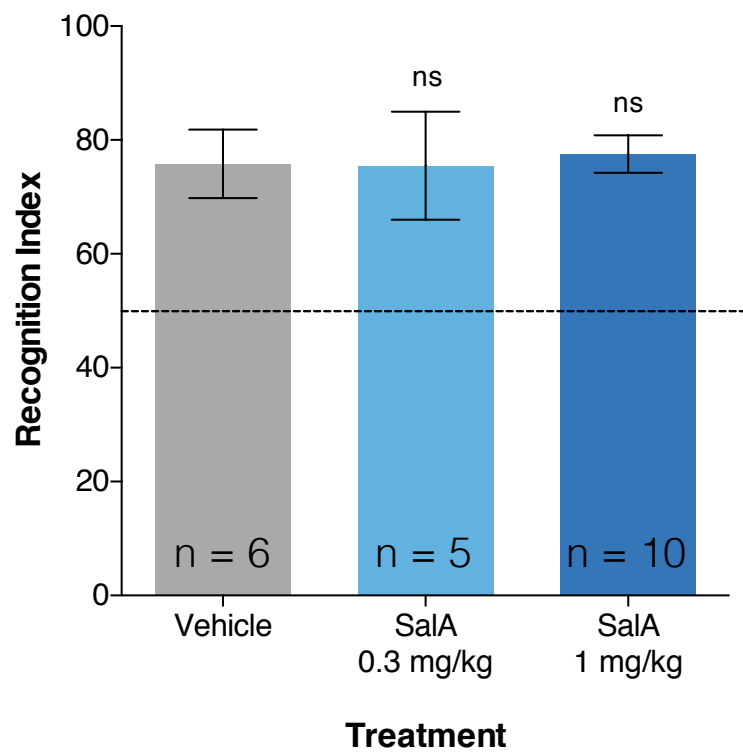

Figure 4.1: Effects of Sal A on RI from the NOR test

Sal A did not produce a significant memory impairment in various NOR protocols. A No significant difference is seen between vehicle and Sal A treatments when using small objects, a 5 min habituation time, and three-phase protocol. B No significant difference seen between vehicle and Sal A treatments when using large objects, a 5 min habituation time, and three-phase protocol. C No significant difference seen between vehicle and Sal A treatments when using large objects, 30 min habituation time, and three-phase protocol. D No significant difference seen between vehicle and Sal A at $0.3 \mathrm{mg} / \mathrm{kg}$ or $1 \mathrm{mg} / \mathrm{kg}$ when using large objects, 20 min habituation time, and two-phase protocol.

Results shown as mean \pm SEM from test phases (A-C, phase III. D, phase II). Dotted line indicates $\mathrm{RI}=50$, equal time spent with both objects.

A-C: un-paired Student t-test, D: one-way ANOVA. 


\subsubsection{U50,488 causes a memory impairment in LTM but not STM}

Because Sal A failed to produce a memory impairment utilising a variety of NOR tests as we expected. Therefore we utilised U50,488, a prototypical KOPr agonist previously shown to impair performance in NOR tests at a $5 \mathrm{mg} / \mathrm{kg}$ ip dose in mice (Schindler et al., 2010).

We chose to follow the protocol outlined by Schindler et al. (2010) with the increased handling time of five days. We also increased habituation to the empty test box for $30 \mathrm{~min}$ a day for three days. A mock injection was also administered on the third habituation day to reduce stress during the test. This protocol was used to test the differences between STM and LTM by altering the delay between the familiarisation and test phases of the NOR test. For STM, a 90 min delay was used, and a $24 \mathrm{~h}$ delay was used to test LTM.

No significant difference was seen between vehicle $(68.5 \pm 4.6(n=13))$ and U50,488 $(10 \mathrm{mg} / \mathrm{kg})(63.4 \pm 6.6(n=13))$ when testing STM $(p=0.5326)$ (figure 4.2A). However, when the time delay between familiarisation and test phases was extended to $24 \mathrm{~h}$ to evaluate LTM impairment, a significant difference in RI was observed (vehicle: $64.0 \pm 4.5(n=16) ; \quad U 50,488: 45.9 \pm 6.0(n=19, p=0.0245))$ (figure 4.2B). Since this protocol produced a significant difference between vehicle and prototypical KOPr agonist U50,488, this methodology was employed to test any long term recognition memory impairment produced by Sal A and novel analogues: Ethy Sal A, Mesyl Sal B, and Bromo Sal A. 
A

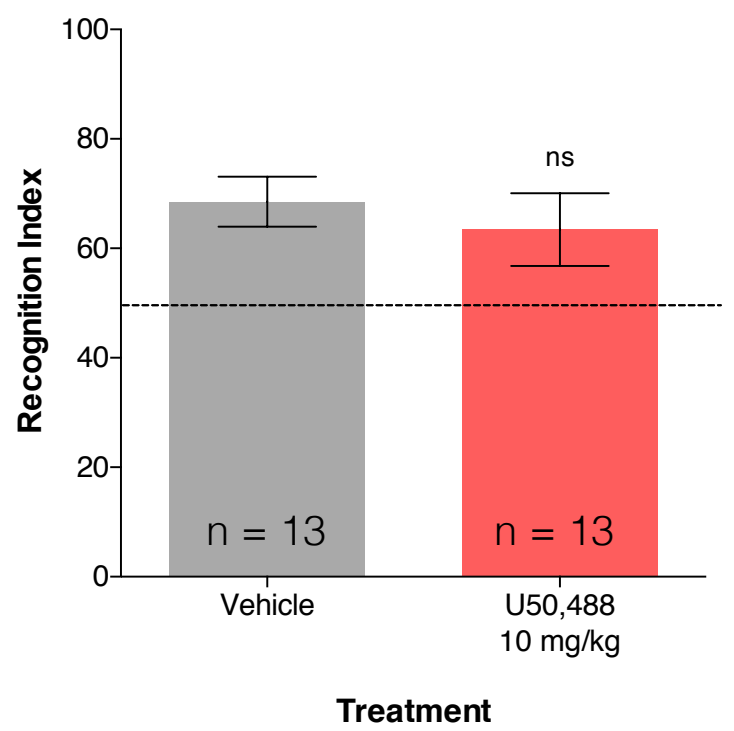

B

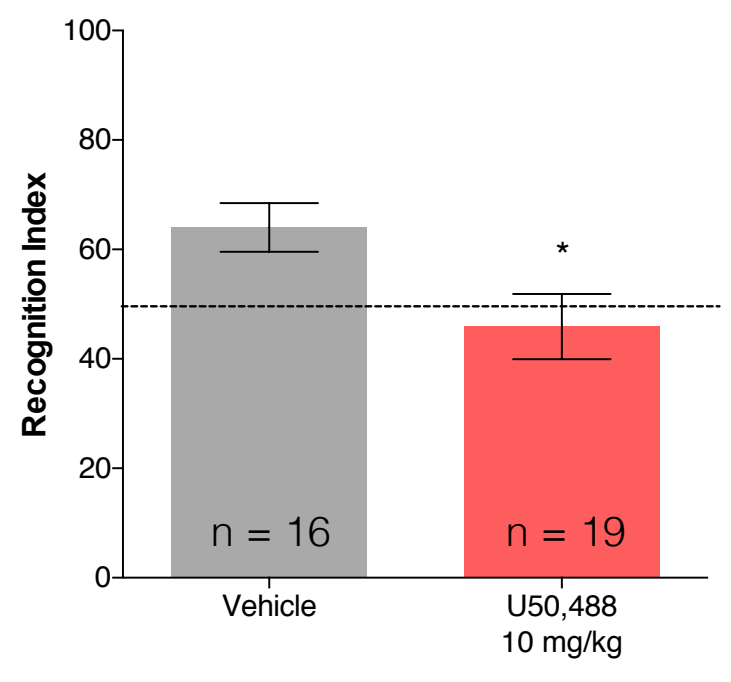

Treatment

Figure 4.2: Effects of U50,488 on STM and LTM

U50,488 produced a significant impairment in long term object recognition memory but not in short term object recognition memory. A When using U50,488 (10 mg/kg) to test STM, no significant memory impairment was seen. B A significant impairment was seen between vehicle and $\mathrm{U} 50,488(10 \mathrm{mg} / \mathrm{kg})$ when investigating long term object recognition memory.

Results shown as mean $\pm \mathrm{SEM}$. Dotted line indicates $\mathrm{RI}=50$, reflects equal time spent with both objects. ${ }^{*} p \leq 0.05$ compared to vehicle. Unpaired Student t-test. 


\subsubsection{Sal $\mathrm{A}$ analogues do not alter long term recognition memory}

Unlike U50,488, Sal A (0.3 and $1 \mathrm{mg} / \mathrm{kg}$ ) and novel analogues Ethy Sal A (0.3 and 1 $\mathrm{mg} / \mathrm{kg}$ ), Mesyl Sal B (0.3 and $1 \mathrm{mg} / \mathrm{kg})$, and Bromo Sal A (1 mg/kg) did not produce a memory impairment in long term recognition memory evaluated in the NOR task.

The Rls between vehicle, U50,488 (10 mg/kg), and each compound were tested with a one-way repeated measures ANOVA followed by a Dunnett's multiple comparisons post-hoc test. When comparing vehicle, U50,488 (10 mg/kg), and Sal A (0.3 mg/kg and 1 $\mathrm{mg} / \mathrm{kg})$, an overall significant difference $(F(2.845,56.90)=3.887, \mathrm{p}=0.0149)$ was seen with the only significant difference between vehicle $(74.6 \pm 3.5)$ and U50,488 $(10 \mathrm{mg} / \mathrm{kg})$ (61.4 \pm 5.3 ). Sal A at 0.3 and $1 \mathrm{mg} / \mathrm{kg}$ saw no significant result compared to vehicle ( 76.7 \pm 3.3 , and $75.0 \pm 3.6$ respectively) (figure $4.3 \mathrm{~A}$ ). An overall significant difference was seen when comparing Ethy Sal A (0.3 and $1 \mathrm{mg} / \mathrm{kg}$ ) with vehicle and U50,488 (10 mg/kg), $F(1.996,39.92)=3.313, p=0.467)$ with the significant difference between vehicle $(74.6 \pm$ $3.5)$ and $U 50,488(10 \mathrm{mg} / \mathrm{kg})(61.4 \pm 5$.). Both Ethy Sal A at $0.3 \mathrm{mg} / \mathrm{kg}$ or $1 \mathrm{mg} / \mathrm{kg}$ saw no significant difference compared to vehicle $(74.5 \pm 2.5$, and $76.5 \pm 2.9$ respectively) (figure 4.3B). When the rats received Mesyl Sal B ( 0.3 or $1 \mathrm{mg} / \mathrm{kg}$ ) treatment $(0.3$ or $1 \mathrm{mg} / \mathrm{kg}$ ), the overall comparison was only trending towards, but was not, significant at the 0.05 level $(F(2.156,43.13)=2.732, p=0.0725)$ (figure $4.3 C)$. Bromo Sal $A(1 \mathrm{mg} / \mathrm{kg})$ also did not produce a significant result when compared to vehicle and U50,488 $(10 \mathrm{mg} / \mathrm{kg})(F(1.683$, $33.66)=2.101, p=0.1447)$. However, Dunnett's multiple comparisons test showed that vehicle $(74.6 \pm 3.5)$ was significantly different from U50,488 $(61.4 \pm 5.3)$ at the 0.05 level (figure 4.3D).

Thus, Sal A (0.3 or $1 \mathrm{mg} / \mathrm{kg}$ ), Ethy Sal A (0.3 or $1 \mathrm{mg} / \mathrm{kg}$ ), or Bromo Sal A ( $1 \mathrm{mg} /$ $\mathrm{kg}$ ), don't show an impairment in long term object recognition memory measured using the NOR task. No sound conclusion can be made about the effect Mesyl Sal B (0.3 or 1 $\mathrm{mg} / \mathrm{kg}$ ) has on the NOR task from the current data. 
A

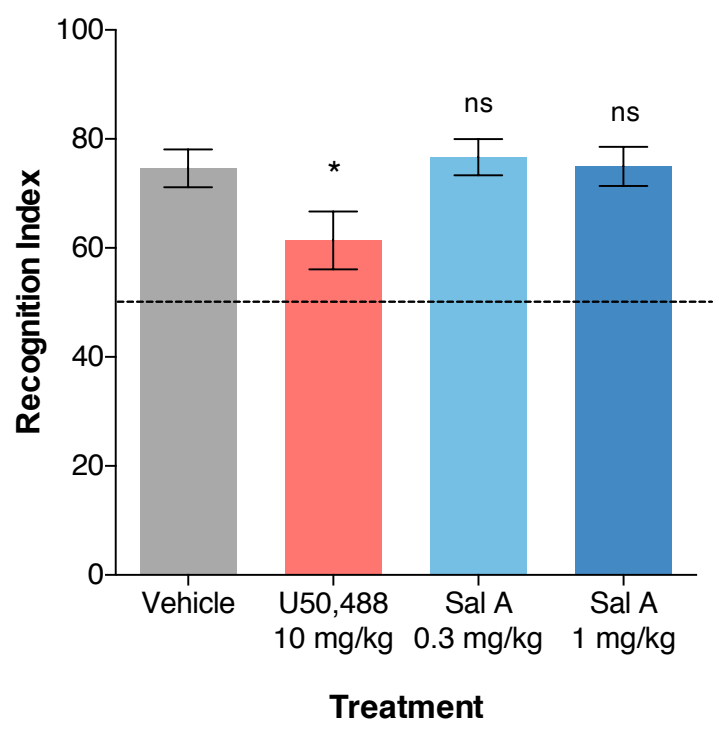

C

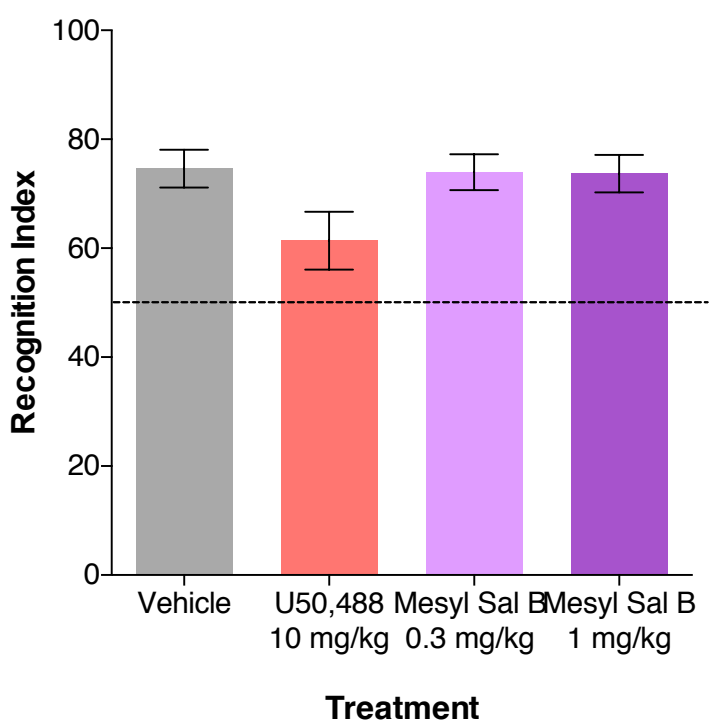

B

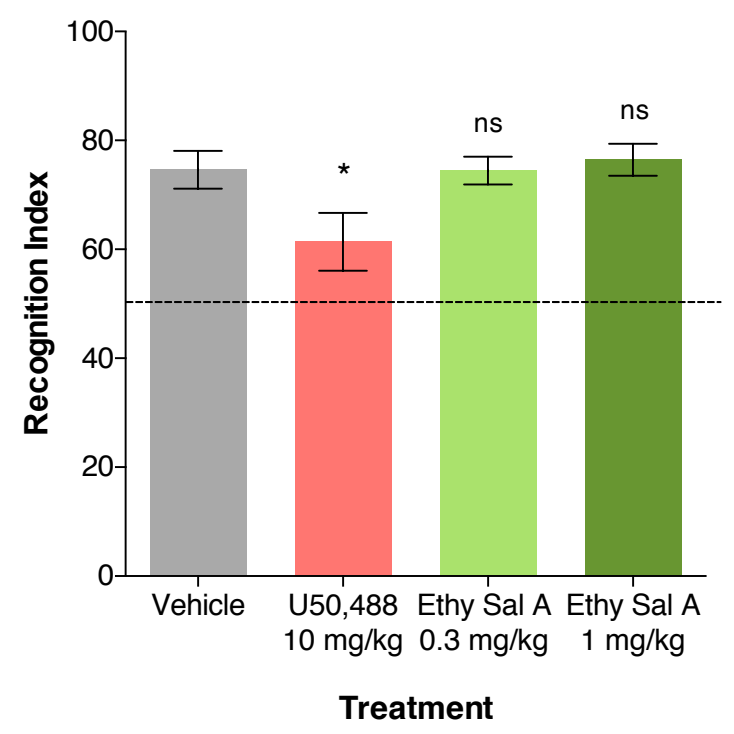

D

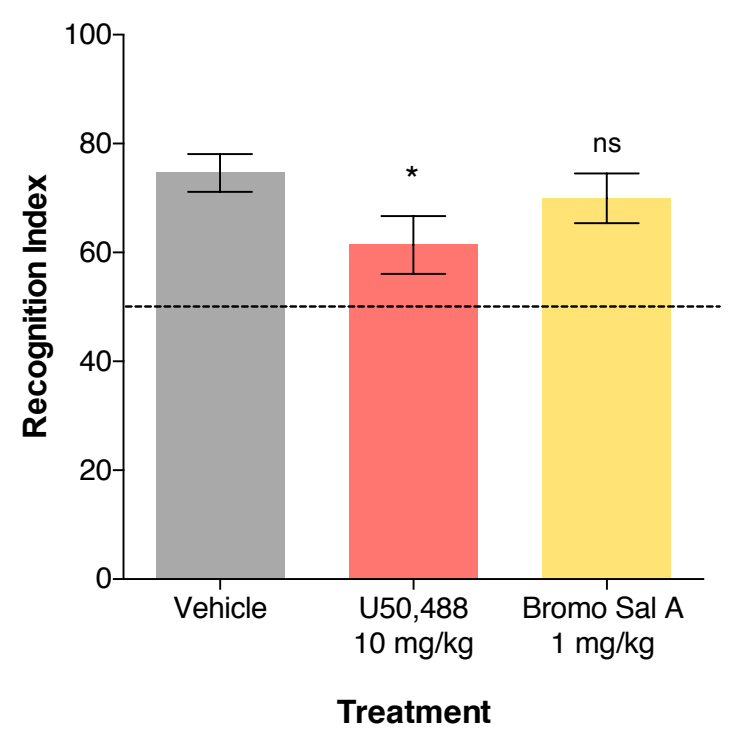

Figure 4.3: Effect of KOPr agonists on RI measured with the NOR task

Unlike U50,488, rats treated with either Sal A (A), Ethy Sal A (B), Mesyl Sal B (C) or Bromo Sal A (D) showed showed no significant change in RI when compared to vehicle.

Results shown as mean \pm SEM. Dotted line indicates $R I=50$, equal time spent with both objects.

${ }^{*} p \leq 0.05$ compared to vehicle. Repeated measures one-way ANOVA with Dunnett's multiple comparisons. $n=21$. 
4.1.6 The long term recognition memory impairment caused by $U 50,488$ is mediated via activation of the $\mathrm{KOPr}$

In order to ensure that the impairment seen in the NOR test with U50,488 were $\mathrm{KOPr}$ mediated effects, a known KOPr antagonist norBNI (10 mg/kg, sc) was administered to 6 rats, $24 \mathrm{~h}$ before a U50,488 injection (10 $\mathrm{mg} / \mathrm{kg}$, ip). Data was compared to U50,488 treatment done within the same rat (within the latin square experimental design). A withinsubjects design was able to be carried out comparing RI between U50,488 trials with norBNI and U50,488 trials.

Analysing the data collected using a repeated measures one-way ANOVA showed a significant difference in RI values from vehicle, U50,488 (10 mg/ $\mathrm{kg}$ ), and norBNI with U50,488 (both $10 \mathrm{mg} / \mathrm{kg})(F(1.862,9.311)=8.394, \mathrm{p}=0.0089)$. Dunnett's multiple comparison tests showed that U50,488 $(46.8 \pm 6.0)$ and norBNI with U50,488 $(85.0 \pm 5.1)$ were significantly different (figure 4.4), showing that the observed memory impairment caused by $\mathrm{U} 50,488$ on long term recognition memory is indeed mediated by the $\mathrm{KOPr}$ system. 


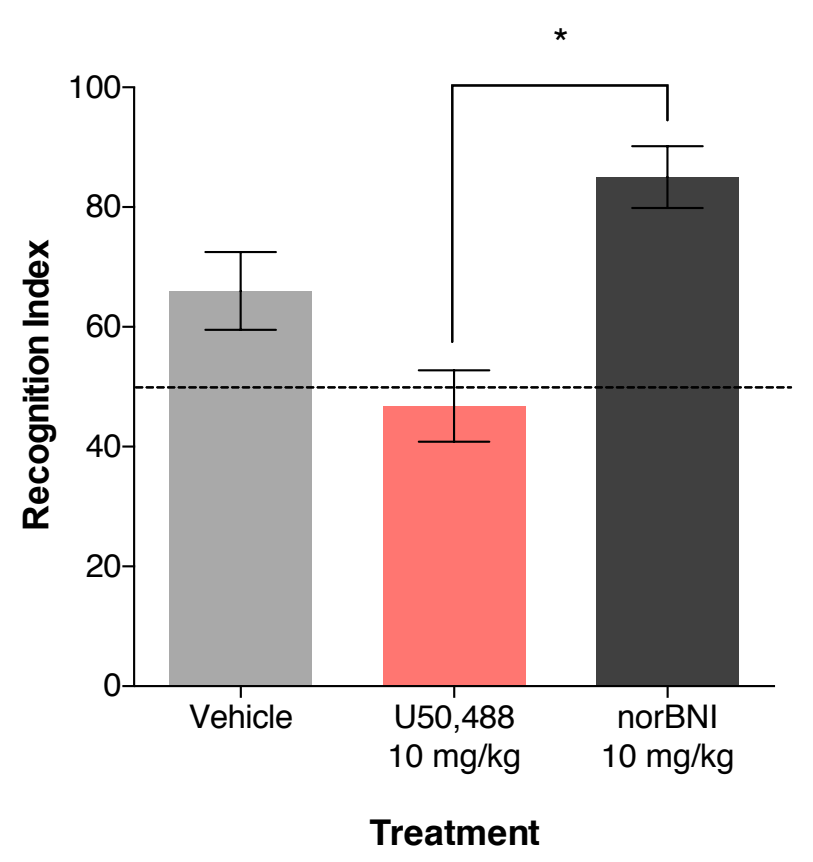

Figure 4.4: norBNI prevented U50,488 mediated long term recognition memory impairment

Male rats pretreated with norBNI $(10 \mathrm{mg} / \mathrm{kg})$ followed by a U50,488 $(10 \mathrm{mg} / \mathrm{kg})$ treatment 24 $\mathrm{h}$ later showed a return to baseline RI after experiencing an impairment caused by U50,488.

Results shown as mean \pm SEM. Dotted line indicates $R I=50$, equal time spent with both objects.

${ }^{*} p \leq 0.05$. Repeated measures one-way ANOVA with Bonferroni's multiple comparisons. $n=6$. 


\subsection{Activation of C-Fos in the perirhinal cortex correlates with U50,488 induced NOR impairment}

\subsection{1 c-Fos counts correlate to $\mathrm{RI}$ values}

Forced swimming in rodents is known to cause c-Fos activation in the cingulate and infralimbic cortices. Therefore, we utilised a 10 min FST to optimise and validate the antibodies used to detect c-Fos immunoreactive cells using IHC staining techniques (see appendix 3).

c-Fos is known to be produced in neurons of the perirhinal cortex in rodents as a sign of novelty. Thus, a correlation between number of c-Fos immunoreactive cells in the perirhinal cortex and RI was expected. Overall, a strong positive correlation was seen between c-Fos counts and RI (figure 4.5A).A Spearman's rank-order correlation test was used to assess this relationship $(r(9)=0.8000, n=11, p=0.0047)$. The number of $c$-Fos positive cells in the perirhinal cortex increased as the RI from the NOR assay increased.

\subsubsection{U50,488 treatment induces activation of c-Fos in the perirhinal cortex}

The $\mathrm{KOPr}$ agonist, $U 50,488(10 \mathrm{mg} / \mathrm{kg}$ ), was utilised to determine whether the number of c-Fos immunopositive cells in the perirhinal cortex were increased compared to vehicle. Stereological techniques were used to quantify the number of c-Fos positive cells in an unbiased manner whilst being representative of the entire perirhinal cortex. An unpaired two-tailed Student's t-test was used to statistically investigate the difference in c-Fos immunoreactive cells between rats treated with $U 50,488$ (10 mg/kg) and vehicle. There was a strong trend towards U50,488 rats (22846 $\pm 904, \mathrm{n}=7$ ) showing reduced c-Fos positive cells compared to vehicle treatment $(26720 \pm 1901, \mathrm{n}=4)(\mathrm{t}(9)=2.104, \mathrm{p}$ $=0.0647$ ) (figure 4.5B). Before testing the novel analogues for c-Fos counts using this protocol, a significant value should be reached. 
A

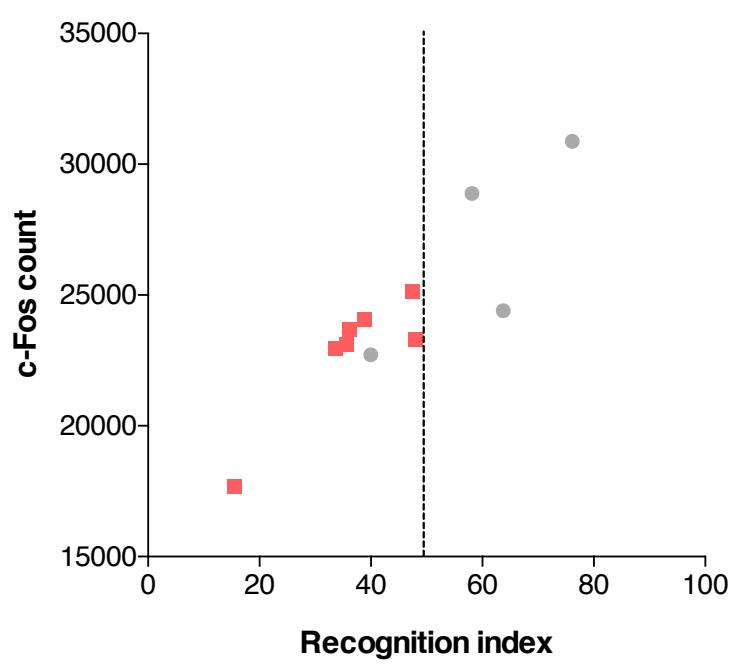

- Vehicle $\quad \mathrm{U} 50,48810 \mathrm{mg} / \mathrm{kg}$
B

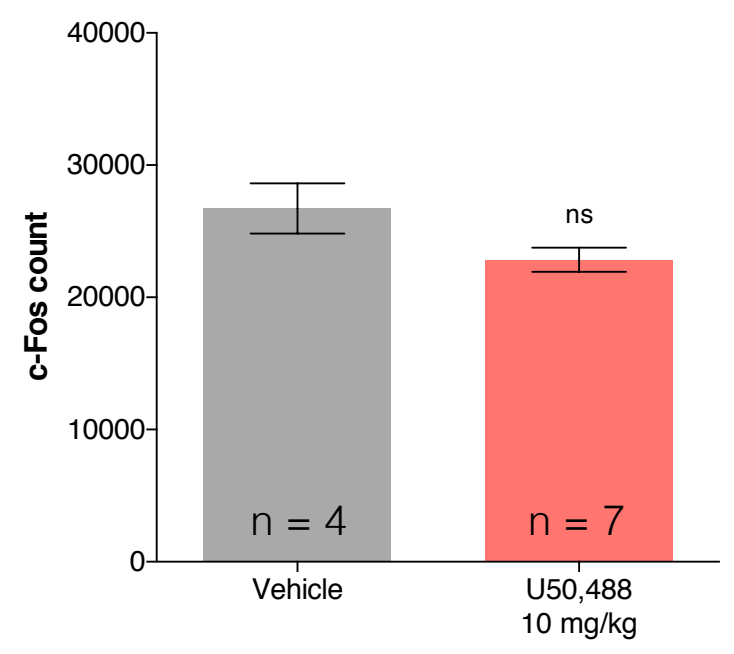

Treatment

Figure 4.5: c-Fos counts in the perirhinal cortex

c-Fos counts in the perirhinal cortex were related to the RI calculated in the NOR task $(\mathbf{A})$ but did not significantly differ between treatments (B).

A Dotted line indicates $\mathrm{RI}=50$, equal time spent with both objects. Spearmans rank-order correlation. $n=11 . p \leq 0.01$

B Results shown as mean \pm SEM. Student's t-test. 


\section{Discussion}

Previous studies by Braida et al. (2011), Carey et al., (2009), and Paris et al., (2011) have shown that Sal A causes a memory impairment in the NOR task in both rats and mice. This is done by habituating rats to a test box with two identical objects in it, then testing if they remember these now familiar objects by replacing one of the objects with something novel in the test phase. By comparing the time spent interacting with each object, a RI can be calculated and used as a measure of memory. Findings that Sal A causes a memory impairment contrasts with our present findings which show no significant change in the $\mathrm{RI}$ between control and Sal A treated groups. It is important to note that the previous studies were carried out in adult male C57BL/6J mice (Carey et al., 2009; Paris et al., 2011) or adult male Wistar rats (Braida et al., 2011) whereas the present study used Sprague-Dawley rats. When Wistar rats were used, Sal A was administered at a dose of $0.160,0.320$, or $0.640 \mathrm{mg} / \mathrm{kg}$ with a group size of 10 in each treatment. The current experiment used Sal $\mathrm{A}$ at a dose of $1 \mathrm{mg} / \mathrm{kg}$ as a positive control and trialled a dose of $0.3 \mathrm{mg} / \mathrm{kg}$ in one of the tests. Since these doses line up with literature values, they are not the likely cause of the discrepancies between what was expected and what was seen. The difference in results between previous and the current study could therefore be due to strain differences in the animals.

Another known KOPr agonist, U50,488, was also shown to produce a memory impairment in the NOR task in adult male C57BL/6 mice (Schindler et al., 2010). The protocol used focused on reducing basal stress levels in the animals prior to evaluation of NOR. To do this, mice were briefly handled for 5 days and received a mock ip injection prior to the experiment. Days 1 through 3 involved habituating the mice to the empty field for 30 min each day. Day 4 was the familiarisation phase. Here, two identical objects were secured on either side of the test box. Mice underwent three 6 min training sessions with 10 min intertrial intervals. On day 5, test day, one of the familiar objects was replaced with a novel object for a 6 min session. Again, the time mice spent with each object was recorded and a $\mathrm{RI}$ calculated. Although this protocol was carried out in mice and, to our 
knowledge, no study has used this in rats, we decided to trial this method in rats using U50,488 (10 mg/kg, ip) as a positive control.

Here, we developed methodologies to enable evaluation of novel Sal A analogues on long term recognition memory using the NOR task. We present evidence that these analogues do not produce a behavioural memory impairment, unlike prototypical KOPr agonist, U50,488. We also develop a protocol for testing $\cup 50,488$ on a cellular level using c-Fos as a marker for novelty. This was considered as the behavioural test may not be sensitive enough to indicate an impairment whereas neuronal studies may indicate subtle changes.

\subsection{NOR protocol optimisation}

In order to recreate the previously shown memory impairment caused by Sal A in the NOR task, we adapted the protocols used previously (Braida et al., 2011; Carey et al., 2009; Paris et al., 2011) in hopes of increasing sensitivity of the task. With the protocols using a three-phase (Carey et al., 2009) and a two-phase (Braida et al., 2011) design, we found a lack of discrimination between novel and familiar objects. This effect could be due to insufficient pre-training (Bevins \& Besheer, 2006). This may result in inducing stress in the animals which in turn could be detrimental to memory (Castellano, Libri, \& Ammassari-Teule, 1988). Lack of interaction with the novel and familiar objects could also be due to interference from the sample-object exposure procedure (Bevins \& Besheer, 2006). One possible method to remove this interference is to increase the time the rats spend with the objects, ensuring sufficient object familiarity. Bevins et al. (2006) put forward a few solutions to increasing object interaction time. These included increased handling, increased habituation to transportation, the use of solid walls in the test box, and increasing the size of the objects used in the test. As the transportation and use of solid walls are not applicable in the current experiment (the test was carried out in the housing facility in a test box with solid walls), object size was first increased to improve object interaction time by requiring more time for total object exploration.

When we modified our procedure to increase the object size from marbles and dice with a diameter or edge length of $1.5 \mathrm{~cm}$ to marbles and dice with a diameter or edge length of $3 \mathrm{~cm}$, we saw an increase in total object-interaction time, but this was still 
insufficient to show significant discrimination between the novel and familiar objects. Bevins et al. (2006) also suggested an increase in environmental familiarisation could increase object interaction time. In increasing habituation to the test box, the environment itself will be familiar to the rat meaning only the novel object will be perceived as novel. In the present study, increasing the habituation time from a single 5 min session to a total of 30 min made up of three 10 min sessions, helped to improve environmental familiarisation, resulting in an increase in object interaction time from $18.2 \pm 3.2 \mathrm{~s}$ to 20.5 $\pm 2.7 \mathrm{~s}$. However, the increase in object-exploration time was still not sufficient to cause robust discrimination between the novel and familiar objects.

Because maximising the total object interaction time did not result in the necessary object discrimination, the protocol design itself was reconsidered. Sal $A$ is known to be metabolised quickly and only has a short elimination half life of $56.6 \pm 24.8$ min in rhesus monkeys (Schmidt et al., 2005). Since the treatment was given before phase I of the three-phase protocol, it is possible that Sal A was no longer active in the rats during phase II or III. This could explain the lack of expected memory impairment from our experiment. If Sal A been fully metabolised before phase II (25 min after injection) or even partway through phase II (up to 35 min after injection), the animal could still have become familiar with the object. Due to this possibility, a two-phase protocol was implemented where Sal A needed to only be active for one $10 \mathrm{~min}$ phase in order for it to exert its effects. Significance between Sal A and vehicle was again not seen as expected (Braida et al., 2011). Possible reasons include the dose used not being high enough or there being strain differences between the animals used in the previously published data and the data collected in the present experiment. That is, adult male Wistar rats were used by Braida et al. (2011) whereas the current study used adult male Sprague-Dawley rats. Strain differences have been identified in anxiety-like behaviour al tests such as open field, the elevated plus maze, and a circular light/dark preference box test (Carr \& Lucki, 2010; van der Staay, Schuurman, van Reenen, \& Korte, 2009). Learning and memory is also affected by the strain of rat used as indicated by Wyss et al. (2000) and Andrews et al. (1995). Comparing Long-Evans, Sprague-Dawley, Wistar, and one inbred strain of rats in a swim maze test and a two-object discrimination test identified that the different strains have different learning and memory capabilities in both spatial and recognition memory. 
Long-Evans rats were found to have the highest learning and memory ability in the tests investigated (Andrews et al., 1995). Thus, the differences seen between previously reported data and the data presented here could be due to strain differences.

Although previously published data showed that Sal A produced a memory impairment using the NOR task (Braida et al., 2011; Carey et al., 2009; Paris et al., 2011), optimisation of three-phase and two-phase protocols failed to show a memory impairment in our laboratory. Therefore, as an additional and alternative positive control, we utilised prototypical KOPr agonist U50,488. This traditional KOPr agonist has been shown to induce memory impairments in both the passive-avoidance task (Castellano et al., 1988) and the NOR task (Schindler et al., 2010) in mice. Although this effect was shown in mice when using the NOR task, we chose to employ a procedure based around that of Schindler et al. (2010) with the main aim of decreasing basal stress levels in the animals. Stress is a confounding factor in memory impairment assays as it is known to itself cause memory impairments in short and long term spatial memory (Conrad, Galea, Kuroda, \& McEwen, 1996; Dominique, Roozendaal, \& McGaugh, 1998; Sandi \& Pinelo-Nava, 2007). By reducing basal stress levels in the animals through an increase in handling and habituation, we hoped to see our positive control produce a memory impairment. It is also known that the mechanisms for STM and LTM within the CNS differ as seen through the same drug affecting STM and LTM in different directions. Different brain regions are also important in different types of memory such as the prefrontal cortex being involved in WM and LTM but not STM, and the amygdala playing an important role in modulation of LTM without being involved in STM (Izquierdo, Medina, Vianna, Izquierdo, \& Barros, 1999). We therefore considered both STM and LTM in hopes to increase the chance of finding a protocol where an expected memory impairment is seen. In doing so, we observed a memory impairment in stress reduced rats when investigating LTM but not in STM tasks. The impairment seen in LTM was very subtle $(p=0.0245)$ with the difference between the positive control and vehicle being slight. The magnitude of memory impairment caused by $\mathrm{U} 50,488(10 \mathrm{mg} / \mathrm{kg})$ is consistent with the magnitude of effects seen with methamphetamine, a compound well-known to negatively affect memory (Herring, Schaefer, Gudelsky, Vorhees, \& Williams, 2008; Kamei et al., 2006; Schroder, O'Dell, \& Marshall, 2003; Vieira-Brock et al., 2015). Herring et al. (2008) investigated the effect of 
methamphetamine on the NOR in rats. Using between 17 and 19 rats per group, a slight yet significant difference was seen ( $p \leq 0.05$ ). The current experiment was carried out with 16 vehicle treated animals and $19 \cup 50,488$ treated animals. As the group sizes and significance level are similar to that seen previously with methamphetamine, the protocol was finalised and used to test the novel analogues.

It is important to note the difference in group sizes used throughout the optimisation tests. In cases where there was high variability between subjects, such as when the smaller objects were used or when the three-phase protocol was employed, multiple trials were not carried out as the high variability made it unlikely to see a significant difference with an increase in group size. However, when the variability was less, such as when investigating LTM and STM, there was potential for the results to show significance so multiple trials were carried out to increase the total group size. A statistical power calculation was carried out once the variability was known to estimate the group size needed for significance (GraphPad software). In cases where the variability was high, the $\mathrm{n}$ size required was too large to be plausible so the specific protocol was disregarded.

\subsection{Testing novel analogues}

After having optimised the NOR protocol so that a memory inducing positive control was observed which indicated the viability of the protocol, we tested Sal A and our novel analogues Ethyl Sal A, Mesyl Sal B, and Bromo Sal A. These analogues were selected due to their high selectivity and potency for the KOPr whilst keeping a low side-effect protocol. We showed that none of these analogues produced an impairment in long term object recognition memory as tested by the NOR task. When designing this experiment, a latin square design was employed such that for each group of three rats, the order in which they received the treatments were different. In doing this, the data from different treatments wouldn't be influenced by which treatment the rats had received the previous week. Using the latin square design validated this and ensured no carry over effects between treatments.

With the design of the experiment, a dose-response curve could be made for Sal A, Ethy Sal A, and Mesyl Say B. Multiple doses for each of these treatments were tested (0.3 and $1 \mathrm{mg} / \mathrm{kg}$ ) as there has been previous evidence on their benefits at both doses used 
including the ability to prevent cocaine reinstatement (Morani et al., 2013). The minimum effective dose for Sal A and Mesyl Sal B is $0.3 \mathrm{mg} / \mathrm{kg}$ as indicated with a cocaine-primedinduced drug-reinstatement model (Riley et al., 2014). For Ethy Sal A, this was found to be $0.1 \mathrm{mg} / \mathrm{kg}$. The lower dose of $0.3 \mathrm{mg} / \mathrm{kg}$ was selected due to being the minimum effective dose. The higher dose of $1 \mathrm{mg} / \mathrm{kg}$ was selected for all treatments as it is more likely to show an effect but has been shown to not cause sedative effects (Carlezon et al., 2006; Morani, Schenk, Prisinzano, \& Kivell, 2012; Riley et al., 2014; Simonson et al., 2015). When looking into dose response curves for these treatments, both Sal $A$ and Mesyl Sal B showed a decrease in RI with an increase in dose whereas Ethy Sal A showed an increase in RI with an increase in dose. Since a lower RI relates to a memory impairment, both Sal A and Mesyl Sal B show a more impaired memory with increased dose albeit this is not a significant trend for either treatment. If these novel treatments were shown to produce a memory impairment, the higher dose would be expected to have a larger memory impairment. However, since this experiment didn't show a memory impairment or enhancement for any of the novel compounds, comment cannot be made on the direction of the recognition index seen with an increase in dose.

A possible explanation for the memory impairment seen is sedation. It is known that KOPr agonists, including U50,488, can produce sedative effects especially at higher doses (Vonvoigtlander, Lahti, \& Ludens, 1983). Although the doses used in the experiment (0.3 and $1 \mathrm{mg} / \mathrm{kg}$ for Sal A, Ethy Sal a, and Mesyl Sal B. $1 \mathrm{mg} / \mathrm{kg}$ for Bromo Sal A) were chosen such that they wouldn't cause sedation. In the present study, the total locomotor activity for each rat was also measured while performing the NOR task, therefore we could evaluate activity via distance traveled. We saw a highly significant difference in total distance travelled when comparing vehicle and $U 50,488$ treated rats, and a slightly significant difference between vehicle and Sal A (1 mg/kg) treated rats (see appendix 4). All other comparisons with vehicle were not significant. The decrease in total distance travelled seen with Sal A did not impact the $\mathrm{Rl}$ as there was not a significant difference in $\mathrm{Rl}$ between Sal $\mathrm{A}$ at $1 \mathrm{mg} / \mathrm{kg}$ and vehicle. However, with U50,488, the decrease in total distance travelled by the rats was greatly decreased compared to vehicle. Since U50,488 was also the only treatment to show a memory impairment based on $\mathrm{RI}$ value, the lowered mobility of the rats could be a confounding factor. That is, the 
lowered activity of the rats would likely be linked to a lowered total interaction with the objects, or with one of the objects. This in turn would affect the RI and come across as a memory impairment during analysis. However, when we consider the total distance travelled by rats who received the known KOPr antagonist norBNI, we see that this is significantly different to vehicle yet not significantly different from U50,488 rats. Taking this with the $\mathrm{RI}$ value of norBNI being significantly greater than $U 50,488$ yet not significantly different from vehicle, we can conclude that the decrease in RI from U50,488 is due to an interaction with memory formation or retrieval and is not influenced by the distance travelled by the animals.

Another possible confounding factor in the current experiment is stress. Although efforts were put into the protocol to minimise basal stress levels in the rats, the U50,488 treatment itself could have induced stress. KOPr agonists have previously been shown to induce aversion in rats (Mucha \& Herz, 1985). U50,488 specifically has been seen to cause a memory impairment in mice measured using the passive-avoidance test (Castellano et al., 1988). This impairment was found to be mediated through the amygdala in response to stress. That is, the $\cup 50,488$ treatment cause a memory impairment through inducing stress in the animal. Similarly, mice who have be subject to stress through the forced swim stress show a decrease in memory measured by the NOR task which is reversed when treated with norBNI (Carey et al., 2009). This indicates that the KOPr system is involved in stress related memory impairment. Thus, if the administration of $\cup 50,488$ to the rats used in the current experiment caused them to become stressed, it could be that KOPr activation inhibits memory by inducing stress. This could be tested for in future experiments by evaluating KOPr agonists on memory in rats who have not been subject to stress and in rats who have be subject to stress, such as through the forced swim test. However, the fact that the novel KOPr agonists did not produce a memory impairment yet have been shown to be more potent than U50,488 at the KOPr, suggests that the memory impairment seen by U50,488 was not stress mediated. Since Sal A has been shown to produce anxiety and stress at 0.3 and $1 \mathrm{mg} / \mathrm{kg}$ in conditioned place aversion (Sufka et al., 2014), yet the same dose did not cause a memory impairment, it is likely that the memory impairment seen from $U 50,488$ is not stress mediated. 


\subsection{Possible mechanisms for memory impairment}

Memory is a complicated brain function with different mechanisms involved in the different types of memory. Part of the pathway involved in long term memory formation and retrieval is cAMP response element binding protein (CREB). This is known to be important in long term memory formation (Yin \& Tully, 1996) and retrieval (Yin et al., 1994). CREB is also induced as part of the KOPr signalling pathway (Bruchas \& Chavkin, 2010). In terms of CREB being involved in memory, experiments in Aplysia, Drosophila, and mice have all come to the conclusion that CREB is important in long-term memory formation with agents that disrupt CREB activity resulting in a blockage of LTM (Silva et al., 1998). However, the specific isoforms of CREB that are induced could have a say in whether LTM is enhanced or impaired. CREB isoforms are either activators or repressors and the effect of CREB on LTM depends on the ratio of these two isoforms. If there is a greater amount of activator isoforms than repressor isoforms, LTM is facilitated and enhanced. However, if there is a greater amount of repressor isoforms than activator isoforms, the formation and retrieval of LTM is prevented (Yin \& Tully, 1996) resulting in a memory impairment. With CREB being induced by KOPr activation (figure 1.1), it is not specified whether the repressor or activator form is more prominent. The current results suggest that the repressor form of CREB is more prominent after activation of the $\mathrm{KOPr}$ system by $\cup 50,488$ which would explain the memory impairment seen.

It is important to note that CREB is only induced through the $\beta$-arrestin recruitment pathway initiated by the $\beta \gamma$ subunit of the GPCR (Bruchas \& Chavkin, 2010) (see figure 1.1). This pathway is the side of the KOPr system that leads to the undesirable side effects such as dysphoria, stress, and aversion. The desired analgesia of KOPr activation is due to early phase ERK1/2 phosphorylation which is part of the G-protein coupled cascade signalling pathway. Bias agonism of the KOPr pathway is possible such that only the $G$ protein pathway is activated, thus there is the possibility to find an analgesic with reduced side effects. As our novel analogues did not produce a memory impairment which is likely to be caused by CREB recruitment, downstream of $\beta$-arrestin pathway activation, it is possible these structurally novel KOPr agonists are bias agonists only activating the desirable G-protein cascade. Current research in our laboratory is looking 
into this possibility on a cellular level, with the current study helping to connect cellular signalling effects to behavioural effects in vivo. Once this connection is created, the longterm aim of this research is to utilise high-throughput cellular screens to evaluate novel agonists for bias and help narrow down which analogues would be beneficial future therapeutics with reduced side effects.

However, the desirable G-protein pathway could also be the pathway to memory impairment. It is known that an increase in concentration of calcium ion or cAMP within the cell activate phosphorylation of CREB (Silva et al., 1998). The G-protein pathway of KOPr activation inhibits the increase of intracellular calcium ion by causing membrane hyperpolarisation, and decreases intracellular cAMP concentration through inhibiting the adenyl cyclase pathway. Both of these effects could restrict CREB activation. Since CREB is important in LTM (Yin, Del Vecchio, Zhou, \& Tully, 1995), the lack of CREB activation could still cause a memory impairment. Thus KOPr agonists could produce a memory impairment through both the $\beta$-arresting recruitment pathway and the G-protein cascade. It is therefore important to continue preclinicalical studyes to identify whether a memory impairment is seen even once a high-throughput screen is established.

\section{4 c-Fos immunohistochemistry}

c-Fos is a transcription factor that can be induced by various factors including tactile experience (Bisler et al., 2002; Filipkowski, Rydz, Berdel, Morys, \& Kaczmarek, 2000), UV radiation (Ghosh, Amstad, \& Cerutti, 1993), and stress (Cullinan et al., 1995). We chose to look into c-Fos activation as a measure of activation of the perirhinal cortex, an area of the rat brain that responds to novelty. An IHC protocol was successfully optimised using forced swim stress as a positive control to induce c-Fos (see appendix 2) such that neurons were selectively stained depending on whether or not they contained c-Fos. When comparing the amount of c-Fos positive cells in the perirhinal cortex with the $\mathrm{RI}$ of the animal, a strong positive correlation was seen such that an increase $\mathrm{Rl}$ corresponded to an increase in number of c-Fos positive neurons. We had hypothesised that the opposite result would occur: that an increase in RI would correspond to a decrease in c-Fos positive cells. This was our hypothesis as c-Fos is induced as the result of novelty (Mendez, Arias, Uceda, \& Arias, 2015). In rats with a memory impairment 
caused by the treatment they received, both the novel and familiar objects would appear novel to them in the test phase. This is due to the disruption of LTM causing them to forget the 'familiar' object. In doing so, memory impaired rats would have been subject to more novelty than their non-memory impaired partners. A memory impaired rats would also be expected to have a lower $\mathrm{Rl}$ as they would theoretically spend approximately the same amount of time with each object whereas rats without a memory impairment would spend more time with the novel object resulting in a higher RI. Thus, we expected a negative correlation between $\mathrm{RI}$ and c-Fos count in the perirhinal cortex although the opposite was observed.

When comparing c-Fos counts between the long term recognition memory impaired U50,488 treated rats and vehicle treated controls, the difference was trending towards statistical significance with the counts for $U 50,488$ being smaller. It is important to note that after carrying out the NOR behavioural task, U50,488 rats were selected for c-Fos analysis if their $\mathrm{RI}$ was less than 50 indicating a memory impairment. Vehicle treated rats were further analysed when the RI was greater than 50 indicating the rats spent more time with the novel than with the familiar, which in turn suggests their memory was not impaired. Based on the c-Fos being induced due to novelty, this would suggest that vehicle rats experience more novelty than $U 50,488$ treated rats, which in turn suggests the vehicle rats experienced a memory impairment whereas the U50,488 rats did not. Based on behavioural data collected from the NOR test itself, this result once more contrasts what was expected.

In conducting this experiment, it was assumed that c-Fos would only be produced as a result of experience to novelty. However, as it can be induced by various other factors, it is possible that these other factors also contributed to its induction such as CREB or $\triangle F$ FosB. All in all, due to the complexity of cellular mechanisms, the vehicle or U50,488 treatments coupled with the NOR task could alter c-Fos expression via mechanisms yet to be discovered.

If c-Fos induction through CREB is considered, a different hypothesis would be proposed. It is known that CREB itself induces c-Fos transcription however, this is not taking into account the activating and repressing forms of CREB. It has been found that the two isoforms of CREB have opposing effects on gene expression (McClung \& Nestler, 
2003). Thus, the specific isoform of CREB which is induced through U50,488 activation of the $\mathrm{KOPr}$ system could lead to c-Fos being unregulated or down regulated. If the activator form of CREB is induced, this will in turn cause an increase in c-Fos levels and the U50,488 treatment would be expected to show an increase in c-Fos count compared to vehicle. However, if it is repressor CREB being induced then c-Fos will be down regulated and the decrease in c-Fos count would be seen as the current experiment showed. This contrasts to what was expected in terms of c-Fos being affected by novelty and reflects on the complexity of cellular pathways.

Further experimentation is needed in order to identify which isoform of CREB is being activated and help understand the complexity of memory pathways. One such way to investigate which isoform is being activated is to induce either the repressor or activator isoform and evaluate how that affects behavioural data. A common method of doing so is through deletion of key CREB isoforms (Carlezon, Duman, \& Nestler, 2005). Engineered mutations of CREB have made it possible to selectively delete dominantnegative isoforms of the protein. If this deletion results in an increase in c-Fos positive cells compared to wild type, it can be concluded that it was a repressor CREB isoform being induced. However, if no change in c-Fos count is observed, it is likely that an activator CREB isoform is being induced.

Furthermore, another protein, $\triangle F$ FosB, is also increased in response to CREB (Renthal \& Nestler, 2009). This protein in turn represses c-Fos (Robison \& Nestler, 2011). This shows a push and pull effect between what happens to c-Fos due to CREB and due to $\triangle F o s B$. The interaction between these two factors is not fully understood however, it has been shown that CREB regulates gene expression after short cocaine treatments whereas $\triangle F$ FosB regulates it more after a longer cocaine treatment (McClung \& Nestler, 2003). Albeit cocaine treatment is not related to the current experiment, this finding regarding the control of gene expression by CREB and $\triangle F O s B$ could shed some light on the induction of c-Fos once the interaction of the two factors is further understood. That is, $\triangle \mathrm{FosB}$ is a later, longer lasting response to $\mathrm{CREB}$. In our experiment, c-Fos counts were taken 90 min after interaction with the novel objects. This time period falls within the earlier stages theoretically meaning that $\triangle$ FosB did not influence the current experiment. 
Alternatively, the unexpected results of c-Fos compared to RI and treatment could be due to errors in the protocol itself rather than effects of the cellular mechanisms involved. One such reason for this could be if the low RI seen with the U50,488 was due to a confounding factor such as lowered locomotion of the rats rather than a memory impairment. Furthermore, experiment bias could have been introduced. This was considered and attempted to be avoided through blinding the photos taken before making c-Fos counts. Bias could not have been introduced when taking the photos due to stereological methods used when capturing them. Thus, bias is unlikely to be the reason behind the unexpected results. Increasing the habituation time to the test box and familiarisation to the familiar object could alter the results. If the test box or the familiar object were still regarded as novel when the rats were tested for a memory impairment, the response of c-Fos to novelty will differ to what is expected. Looking into the IHC staining protocol instead of the behavioural aspect, the unexpected results could be due to unspecific staining of the antibodies used. This was theoretically avoided when optimising the protocol using forced swim stress as a positive control. It should also be noted that testing the effects a treatment has on c-Fos expression after the NOR task has not yet been published. The current experiment was therefore novel and decisions around each step were made based purely on current theory. The current experiment could therefore be a potential avenue into discovering more detail about the interaction of KOPr agonists, memory impairment, and c-Fos or more likely, it could be that the joining of c-Fos IHC with the NOR task should be carefully rediscovered and a slightly different protocol developed.

In terms of using this c-Fos IHC to test novel analogues for a potential memory impairment, results seen with a positive control such as $U 50,488$ must be further investigated. Ensuring that the results seen are reproducible by repeating the experiment will indicate whether or not the effects seen were due to cellular mechanisms or due to other confounding factors. Considering what is known currently, it is expected that the novel analogues would produce high levels of c-Fos, similar to what was seen with vehicle runs. 


\subsection{Future directions}

To further investigate whether these novel objects have an effect on learning and memory, additional types of memory tests should also be investigated. This could be done by altering the NOR task used slightly or by looking into other behavioural tasks. In terms of altering the current procedure to look into a different memory type, if one of the familiar objects is moved between familiarisation and test instead of changing it for a novel object, the task will be looking into spatial memory. This is controlled mainly by the hippocampus and it would be beneficial to test whether the KOPr agonists impair this type of memory, both short-term and long-term, due to the high density of kappa receptors themselves within the hippocampus. Another memory tasks that could be considered would be the $\mathrm{Y}$ - or T- maze. These two tests look into spontaneous alternation and spatial memory. Previous studies have shown that the endogenous KOPr agonist, dynorphin, improves spontaneous alternation measured in mice using the Y-maze (Itoh, Ukai, \& Kameyama, 1994). It would be interesting to investigate if this effect carries over into rats and with exogenous KOPr agonists. The current NOR protocol can also be altered to look into spatial memory in lieu of recognition memory by moving a familiar object instead of introducing a novel object in the test phase. Combining this possible methodology with results from the Y- or T- maze, both behavioural tests looking into spatial memory, would produce a robust data set which would allow for sound conclusions to be extrapolated and stated as being due to the memory impairment and not specific factors of the behavioural task. For any memory test used in the future, various types of memory should be considered such as WM, STM, and LTM simply by altering the time between the learning and test phases.

Other beneficial studies to carry out regarding the side effect profile of our novel analogues include comparing higher doses of the treatments, looking into differences between males and females, using a different animal model such as mice to investigate how universal the observed results are across species, and also investigating whether chronic KOPr agonist activation results in differences compared to thee acute treatment used in the current experiment. This is important as if a KOPr agonist does make it through clinical trials and is implemented as a therapeutic, its use will likely be chronic. Thus information regarding side effects after chronic treatment is crucial. Also, 
administering the treatment at different times throughout the experiment could allow us to look into different aspects of the memory process such as memory formation, consolidation, or retrieval. For example, if the effect of a treatment on memory formation is wished to be investigated, administering the treatment before the familiarisation phase with the objects will allow this to occur.

For the IHC, the next step would be to increase the number of subjects in the vehicle treatment group to see if significance can be reached. Further studies on the mechanism behind how U50,488 impairs recognition memory could also be beneficial. Possible methods for this include Western blots and real time quantitative PCR. Once the mechanism for how KOPr agonists affect learning and memory is known, their possible therapeutic uses could be extrapolated more easily with more compounds being tested throughout.

\subsection{Conclusions.}

Unlike the prototypical KOPr agonists U50,488, neither Sal A (0.3 and $1 \mathrm{mg} / \mathrm{kg}$ ), nor the novel analogues Ethy Sal A (0.3 and $1 \mathrm{mg} / \mathrm{kg}$ ), Mesyl Sal B (0.3 and $1 \mathrm{mg} / \mathrm{kg}$ ), and Bromo Sal A (1 mg/kg), impaired memory in the NOR task. Therefore, long term object recognition memory is not impaired by Sal $A$ analogues, showing reduced negative sideeffects compared with tradition KOPr agonists. 


\section{Supplementary information}

\subsection{Appendix 1: Solutions}

10X Phosphate buffered saline (PBS) ph7.4

Concentration $\quad \mathrm{g} / \mathrm{L}$

$\begin{array}{lll}\mathrm{NaCl} & 1.4 \mathrm{M} & 80\end{array}$

$\begin{array}{lll}\mathrm{KCl} & 26.8 \mathrm{mM} & 2.0\end{array}$

$\mathrm{Na}_{2} \mathrm{HPO}_{4} \quad 81.0 \mathrm{mM} \quad 26.8$

$\begin{array}{lll}\mathrm{KH}_{2} \mathrm{PO}_{4} & 14.7 \mathrm{mM} & 2.4\end{array}$

4\% Paraformaldehyde in PBS pH7.4

PFA $\quad 40 \mathrm{~g}$

$1 \times$ PBS $1 \mathrm{~L}$

Preheat about $900 \mathrm{~mL}$ of $1 \times$ PBS to $50{ }^{\circ} \mathrm{C}$. Add in the $40 \mathrm{~g}$ PFA powder. Add drops of $\mathrm{NaOH}$ until the PFA dissolves. Make up to $1 \mathrm{~L}$ with $1 \times$ PBS. Let cool and $\mathrm{pH}$ to 7.4

$30 \%$ sucrose solution

Sucrose $\quad 3 g$

$1 \times$ PBS $\quad 10 \mathrm{~mL}$

\section{$0.2 \%$ PBS-T}

Triton X-100 $100 \mu \mathrm{L}$

$1 \times$ PBS $\quad 49.9 \mathrm{~mL}$ 


\subsection{Appendix 2: Perfusion protocol}

After euthanising the rat, a midline incision was made through the abdomen along the length of the diaphragm. The connective tissue at the base of the diaphragm was cut followed by each side of the ribs allowing the ribcage to be pulled up exposing the heart. A small incision in the right atrium of the rats heart was made to allow blood to escape. A needle (18 gauge) was inserted into the left ventricle and clamped into place. 1x PBS was pumped through the rat using a peristaltic pump fitted with silastic tubing at a rate of $11 \mathrm{~mL} / \mathrm{min}$. Once the perfusate ran clear of blood and the liver lightened in colour (200 $300 \mathrm{~mL}$ ), the PBS was exchanged for $4 \%$ PFA and the rat was perfused until it was stiff to fix the brain tissue. 


\subsection{Appendix 3: IHC optimisation data}

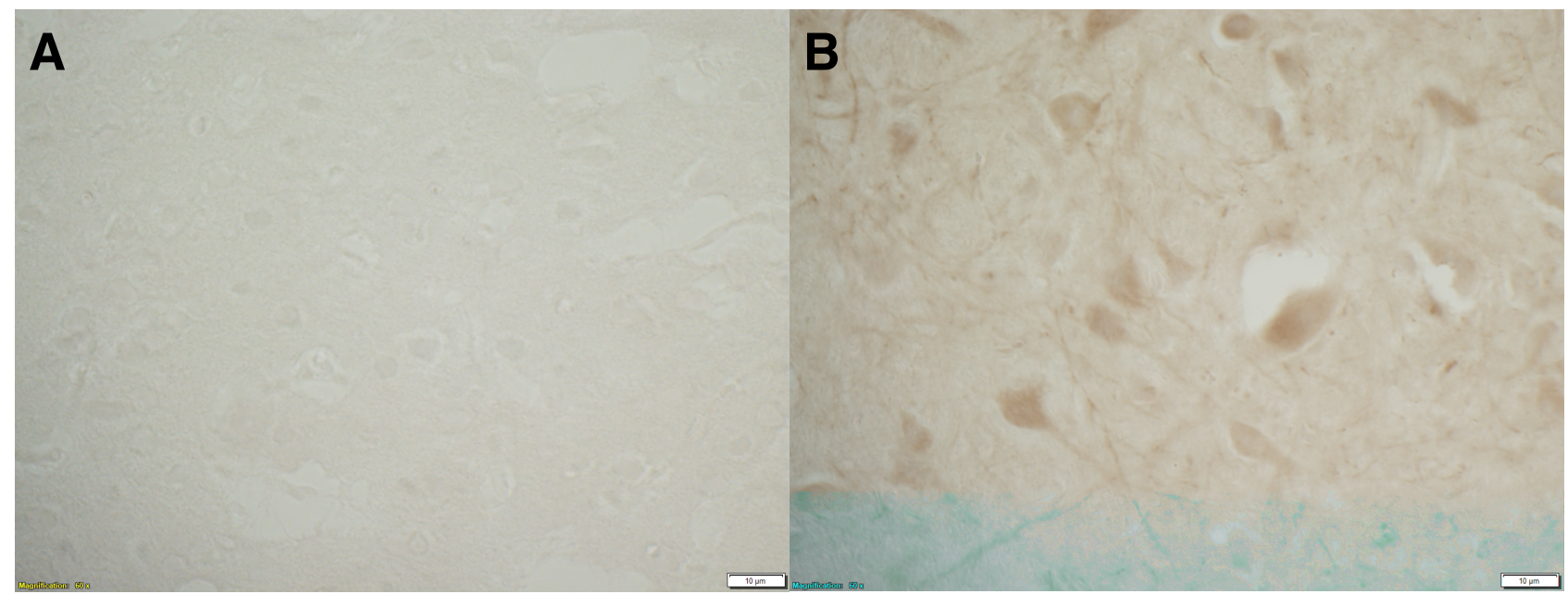

Figure 6.1: Example photos of IHC optimisation

Photos taken from rat $\mathrm{Cg} 30$ min after 10 min FST. A Primary antibody control. IHC staining protocol carried out without any primary antibody to ensure staining is specific. B Example section showing positively stained c-Fos immunoreactive cells.

$60 x$ magnification. 


\subsection{Appendix 4: Locomotor data}

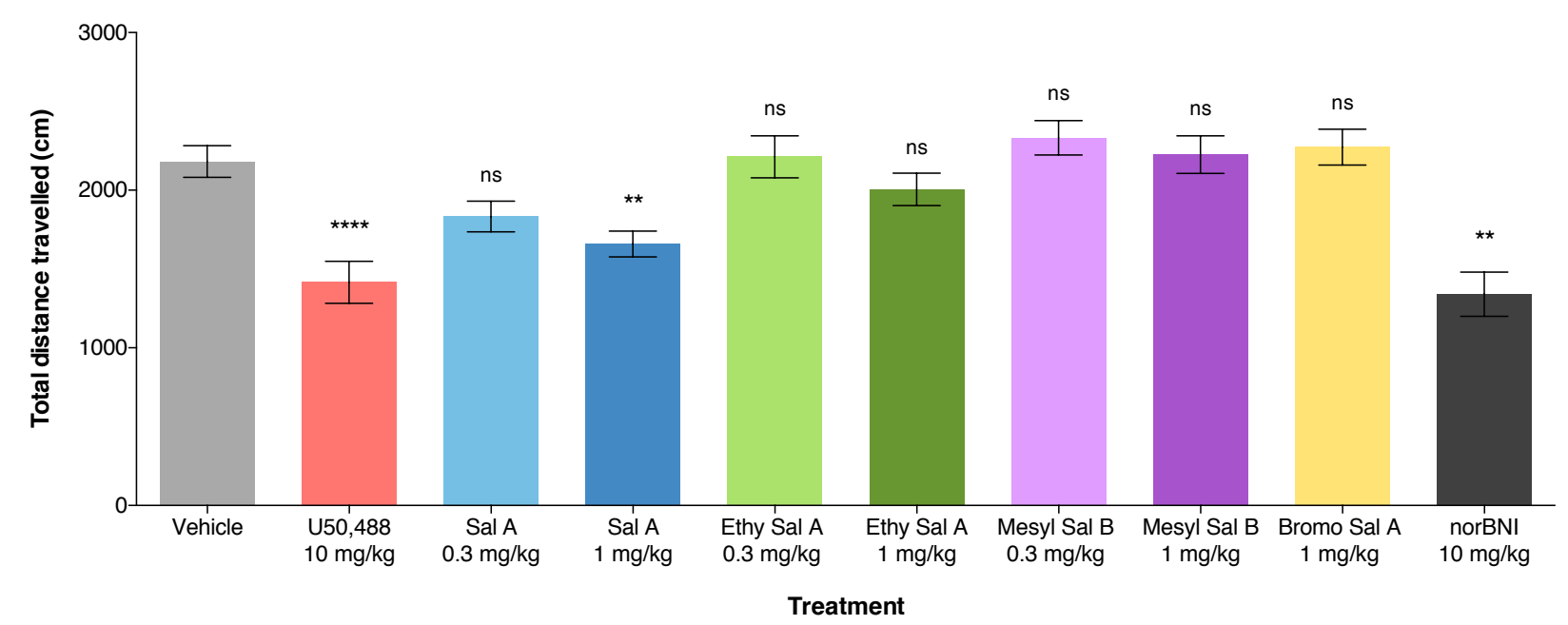

Figure 6.2: KOPr treatments affecting total distance travelled

Rats treated with prototypical agonist U50,488 (10 mg/kg), Sal A (1 mg/kg), or KOPr antagonist norBNI $(10 \mathrm{mg} / \mathrm{kg})$ showed a decrease in total distance travelled during the 6 min test phase of the NOR task indicating a sedative effect of the treatments.

${ }^{* \star} p<0.01,{ }^{\star * \star *} p<0.0001$ compared to vehicle. One-way ANOVA with Bonferroni's multiple comparisons test. $\mathrm{n}=21$. 


\section{Literature cited}

Aldrich, \& McLaughlin. (2009). Peptide kappa opioid receptor ligands: potential for drug development. AAPS J, 11(2), 312-322.

Andrews, Jansen, Linders, Princen, \& Broekkamp. (1995). Performance of four different rat strains in the autoshaping, two-object discrimination, and swim maze tests of learning and memory. Physiology \& Behavior, 57(4), 785-790.

Antunes, \& Biala. (2012). The novel object recognition memory: neurobiology, test procedure, and its modifications. Cognitive Processing, 13(2), 93-110.

Barnes, Holmes, Primeaux, York, \& Bray. (2006). Increased expression of mu opioid receptors in animals susceptible to diet-induced obesity. Peptides, 27(12), 3292-3298.

Bear, Connors, \& Paradiso. (2007). Neuroscience (Vol. 2): Lippincott Williams \& Wilkins.

Beguin, Potter, DiNieri, Munro, Richards, Paine, .. . Xu. (2008). N-Methylacetamide Analog of Salvinorin A: A Highly Potent and Selective k-Opioid Receptor Agonist with Oral Efficacy. Journal of Pharmacology and Experimental Therapeutics, 324(1), 188-195.

Belcheva, Clark, Haas, Serna, Hahn, Kiss, \& Coscia. (2005). $\mu$ and K opioid receptors activate ERK/MAPK via different protein kinase $\mathrm{C}$ isoforms and secondary messengers in astrocytes. Journal of Biological Chemistry, 280(30), 27662-27669.

Bellchambers, Chieng, Keay, \& Christie. (1998). Swim-stress but not opioid withdrawal increases expression of c-fos immunoreactivity in rat periaqueductal gray neurons which project to the rostral ventromedial medulla. Neuroscience, 83(2), 517-524.

Bevins, \& Besheer. (2006). Object recognition in rats and mice: a one-trial nonmatching-to-sample learning task to study 'recognition memory'. Nature Protocols, 1(3), 1306-1311.

Bisler, Schleicher, Gass, Stehle, Zilles, \& Staiger. (2002). Expression of c-Fos, ICER, Krox-24 and JunB in the whisker-to-barrel pathway of rats: time course of induction upon whisker stimulation by tactile exploration of an enriched environment. Journal of Chemical Neuroanatomy, 23(3), 187-198. 
Borniger, \& Hesp. (2016). Enhancing remyelination through a novel opioid-receptor pathway. Journal of Neuroscience, 36(47), 11831-11833.

Bourtchuladze, Frenguelli, Blendy, Cioffi, Schutz, \& Silva. (1994). Deficient long-term memory in mice with a targeted mutation of the cAMP-responsive element-binding protein. Cell, 79(1), 59-68.

Braida, Capurro, Zani, Rubino, Vigano, Parolaro, \& Sala. (2009). Potential anxiolyticand antidepressant-like effects of salvinorin $A$, the main active ingredient of Salvia divinorum, in rodents. British Journal of Pharmacology, 157(5), 844-853.

Braida, Donzelli, Martucci, Capurro, \& Sala. (2011). Learning and memory impairment induced by salvinorin A, the principal ingredient of Salvia divinorum, in wistar rats. International Journal of Toxicology, 30(6), 650-661.

Bruchas, \& Chavkin. (2010). Kinase cascades and ligand-directed signaling at the kappa opioid receptor. Psychopharmacology (Berl), 210(2), 137-147.

Bruchas, Land, Aita, Xu, Barot, Li, \& Chavkin. (2007). Stress-induced p38 mitogenactivated protein kinase activation mediates k-opioid-dependent dysphoria. Journal of Neuroscience, 27(43), 11614-11623.

Bruijnzeel. (2009). kappa-Opioid receptor signaling and brain reward function. Brain Research Reviews, 62(1), 127-146.

Burford, Traynor, \& Alt. (2015). Positive allosteric modulators of the $\mu$-opioid receptor: a novel approach for future pain medications. British Journal of Pharmacology, 172(2), 277-286.

Carey, Lyons, Shay, Dunton, \& McLaughlin. (2009). Endogenous kappa opioid activation mediates stress-induced deficits in learning and memory. Journal of Neuroscience, 29(13), 4293-4300.

Carlezon, Beguin, DiNieri, Baumann, Richards, Todtenkopf, . . . Cohen. (2006). Depressive-like effects of the kappa-opioid receptor agonist salvinorin A on behavior and neurochemistry in rats. Journal of Pharmacology and Experimental Therapeutics, 316(1), 440-447.

Carlezon, Duman, \& Nestler. (2005). The many faces of CREB. Trends in Neurosciences, 28(8), 436-445. 
Carr, \& Lucki. (2010). Comparison of the kappa-opioid receptor antagonist DIPPA in tests of anxiety-like behavior between Wistar Kyoto and Sprague Dawley rats. Psychopharmacology, 210(2), 295-302.

Castellano, Libri, \& Ammassari-Teule. (1988). The amygdala mediates the impairing effect of the selective $\mathrm{K}$-opioid receptor agonist $\mathrm{U}-50,488$ on memory in CD1 mice. Behavioural Brain Research, 30(3), 259-263.

Center. (2011). Pharmacology of opioids in the treatment of chronic pain syndromes. Pain Physician, 14, E343-E360.

Cohen, \& Squire. (1980). Preserved learning and retention of pattern-analyzing skill in amnesia: Dissociation of knowing how and knowing that. Science, 210(4466), 207-210.

Conrad, Galea, Kuroda, \& McEwen. (1996). Chronic stress impairs rat spatial memory on the $Y$ maze, and this effect is blocked by tianeptine treatment. Behavioral Neuroscience, 110(6), 1321.

Crowley, \& Kash. (2015). Kappa opioid receptor signaling in the brain: Circuitry and implications for treatment. Progress in Neuropsychopharmacology and Biological Psychiatry, 62, 51-60.

Cullinan, Herman, Battaglia, Akil, \& Watson. (1995). Pattern and time course of immediate early gene expression in rat brain following acute stress. Neuroscience, 64(2), 477-505.

Dash, Hochner, \& Kandel. (1990). Injection of the cAMP-responsive element into the nucleus of Aplysia sensory neurons blocks long-term facilitation. Nature, 345(6277), 718-721.

Daumas, Betourne, Halley, Wolfer, Lipp, Lassalle, \& Francés. (2007). Transient activation of the CA3 Kappa opioid system in the dorsal hippocampus modulates complex memory processing in mice. Neurobiology of Learning and Memory, 88(1), 94-103.

Deehan, McKinzie, Carroll, McBride, \& Rodd. (2012). The long-lasting effects of JDTic, a kappa opioid receptor antagonist, on the expression of ethanol-seeking behavior and the relapse drinking of female alcohol-preferring $(P)$ rats. Pharmacology, Biochemistry and Behaviour, 101(4), 581-587. 
Dogra, \& Yadav. (2015). Biased agonism at kappa opioid receptors: Implication in pain and mood disorders. European Journal of Pharmacology, 763, 184-190.

Dominique, Roozendaal, \& McGaugh. (1998). Stress and glucocorticoids impair retrieval of long-term spatial memory. Nature, 394(6695), 787-790.

Du, Duan, Wei, Cai, Chai, Lv, ... Xie. (2016). Kappa opioid receptor activation alleviates experimental autoimmune encephalomyelitis and promotes oligodendrocytemediated remyelination. Nature Communications, 7, 11120.

Duncan, Johnson, \& Breese. (1993). Topographic patterns of brain activity in response to swim stress: assessment by 2-deoxyglucose uptake and expression of Foslike immunoreactivity. Journal of Neuroscience, 13(9), 3932-3943.

Duncan, Knapp, Johnson, \& Breese. (1996). Functional classification of antidepressants based on antagonism of swim stress-induced fos-like immunoreactivity. Journal of Pharmacology and Experimental Therapeutics, 277(2), 1076-1089.

Durán-Prado, Malagón, Gracia-Navarro, \& Castaño. (2008). Dimerization of G protein-coupled receptors: new avenues for somatostatin receptor signalling, control and functioning. Molecular and Cellular Endocrinology, 286(1), 63-68.

Feng, He, Yang, Chao, H Lazarus, \& Xia. (2012). Current research on opioid receptor function. Current Drug Targets, 13(2), 230-246.

Filipkowski, Rydz, Berdel, Morys, \& Kaczmarek. (2000). Tactile experience induces c-fos expression in rat barrel cortex. Learning \& Memory, 7(2), 116-122.

Fine. (2008). The Britannica Guide to The Brain: A guided tour of the brain-mind, memory, and intelligence: Encyclopaedia Britannica.

Ghelardini, Galeotti, Bartolini, Anzini, Cappelli, \& Vomero. (2001). Antiamnesic Effect of the Two Novel k-Opioid Agonists, VA 100 and VA 101, in the Mouse Passive Avoidance Test. Drug Development and Research, 54, 12-18.

Ghosh, Amstad, \& Cerutti. (1993). UVB-induced DNA breaks interfere with transcriptional induction of c-fos. Molecular and Cellular Biology, 13(11), 6992-6999.

Goldenberg. (2012). Multiple sclerosis review. PT, 37(3), 175-184.

Goldstein, \& Ghazarossian. (1980). Immunoreactive dynorphin in pituitary and brain. Proceedings of the National Academy of Sciences, 77(10), 6207-6210. 
Grella, Funk, Coen, Li, \& Le. (2014). Role of the kappa-opioid receptor system in stress-induced reinstatement of nicotine seeking in rats. Behavioural Brain Research, 265, 188-197.

Gupta, Décaillot, \& Devi. (2006). Targeting opioid receptor heterodimers: strategies for screening and drug development. The AAPS journal, 8(1), E153-E159.

Gupta, Upmanyu, \& Vashist. (2012). Various Animal Models To Check Learning And Memory-A Review: LAP LAMBERT Academic Publishing.

Hajrasouliha, Tavakoli, Jabehdar-Maralani, Ebrahimi, Shafaroodi, Mirkhani, . . . Dehpour. (2005). Cholestatic liver disease modulates susceptibility to ischemia/ reperfusion-induced arrhythmia, but not necrosis and hemodynamic instability: the role of endogenous opioid peptides. Journal of Hepatology, 43(3), 491-498.

Harding, Tidgewell, Byrd, Cobb, Dersch, Butelman, . . . Prisinzano. (2005). Neoclerodane diterpenes as a novel scaffold for mu opioid receptor ligands. Journal of Medicinal Chemistry, 48(15), 4765-4771.

Harlan, \& Garcia. (1998). Drugs of abuse and immediate-early genes in the forebrain. Molecular Neurobiology, 16(3), 221-267.

Herring, Schaefer, Gudelsky, Vorhees, \& Williams. (2008). Effect of (+)methamphetamine on path integration learning, novel object recognition, and neurotoxicity in rats. Psychopharmacology, 199(4), 637-650.

Hiramatsu, \& Hoshino. (2004). Involvement of kappa-opioid receptors and sigma receptors in memory function demonstrated using an antisense strategy. Brain Research, $1030(2), 247-255$.

Hiramatsu, Hoshino, Kameyama, \& Nabeshima. (2002). Involvement of K-opioid and $\sigma$ receptors in short-term memory in mice. European Journal of Pharmacology, 453(1), 91-98.

Hiramatsu, Hyodo, \& Kameyama. (1997). U-50,488 H, a selective kappa opoid receptor agonist, ameliorates memory impairments induced by muscarinic autoreceptor agonist, carbachol in mice. Neuroscience Letters, 236(1), 45-48.

Hiramatsu, \& Kameyama. (1998). Roles of kappa-opioid receptor agonists in learning and memory impairment in animal models. Methods and Findings in Experimental and Clinical Pharmacology, 20(7), 595-599. 
Hiramatsu, Mizuno, \& Kanematsu. (2006). Pharmacological characterization of the ameliorating effect on learning and memory impairment and antinociceptive effect of KT-95 in mice. Behavioural Brain Ressearch, 167(2), 219-225.

Hiramatsu, Mori, Murasawa, \& Kameyama. (1996). Improvement by dynorphin A (113) of galanin-induced impairment of memory accompanied by blockade of reductions in acetylcholine release in rats. British Journal of Pharmacology, 118(2), 255-260.

Hiramatsu, Murasawa, Nabeshima, \& Kameyama. (1998). Effects of U-50,488 H on scopolamine-, mecamylamine-and dizocilpine-induced learning and memory impairment in rats. Journal of Pharmacology and Experimental Therapeutics, 284(3), 858-867.

Hughes, \& Dragunow. (1995). Induction of immediate-early genes and the control of neurotransmitter-regulated gene expression within the nervous system. Pharmacological Reviews, 47(1), 133-178.

legorova, Fisyunov, \& Krishtal. (2010). G-protein-independent modulation of P-type calcium channels by $\mu$-opioids in Purkinje neurons of rat. Neuroscience Letters, 480(2), 106-111.

Institute of Medicine. (2011). Relieving pain in America: A blueprint for transforming prevention, care, education, and research.

Itoh, Ukai, \& Kameyama. (1994). Dynorphin A-(1-13) potently improves the impairment of spontaneous alternation performance induced by the mu-selective opioid receptor agonist DAMGO in mice. Journal of Pharmacology and Experimental Therapeutics, 269(1), 15-21.

Izquierdo, Medina, Vianna, Izquierdo, \& Barros. (1999). Separate mechanisms for short-and long-term memory. Behavioural Brain Research, 103(1), 1-11.

John, French, \& Erlichman. (2006). The antinociceptive effect of salvinorin A in mice. European Journal of Pharmacology, 545(2-3), 129-133.

Jordan, Cvejic, \& Devi. (2000). Opioids and their complicated receptor complexes. Neuropsychopharmacology, 23, S5-S18.

Kam, Chan, \& Wong. (2004). K-opioid receptor signals through Src and focal adhesion kinase to stimulate c-Jun N-terminal kinases in transfected COS-7 cells and human monocytic THP-1 cells. Journal of Pharmacology and Experimental Therapeutics, 310(1), 301-310. 
Kamei, Nagai, Nakano, Togan, Takayanagi, Takahashi, . . . Takuma. (2006). Repeated methamphetamine treatment impairs recognition memory through a failure of novelty-induced ERK1/2 activation in the prefrontal cortex of mice. Biological psychiatry, 59(1), 75-84.

Kelsey, Verhaak, \& Schierberl. (2015). The kappa-opioid receptor antagonist, norbinaltorphimine (nor-BNI), decreases morphine withdrawal and the consequent conditioned place aversion in rats. Behavioural Brain Research, 283, 16-21.

Knoll, \& Carlezon. (2010). Dynorphin, stress, and depression. Brain Research, 1314, 56-73.

Ko, \& Husbands. (2009). Effects of atypical kappa-opioid receptor agonists on intrathecal morphine-induced itch and analgesia in primates. Journal of Pharmacology and Experimental Therapeutics, 328(1), 193-200.

Koob, \& Le Moal. (2001). Drug addiction, dysregulation of reward, and allostasis. Neuropsychopharmacology, 24(2), 97-129.

Kristiansen, \& Nyengaard. (2012). Digital stereology in neuropathology. APMIS, 120(4), 327-340.

Lahti, VonVoigtlander, \& Barsuhn. (1982). Properties of a selective kappa agonist, U-50,488 H. Life sciences, 31(20), 2257-2260.

Li, Luo, Krupnick, Benovic, \& Liu-Chen. (1999). U50, 488H-induced Internalization of the Human k Opioid Receptor Involves a $\beta$-Arrestin-and Dynamin-dependent Mechanism. Journal of Biological Chemistry, 274(17), 12087-12094.

Liu-Chen. (2004). Agonist-induced regulation and trafficking of $\mathrm{k}$ opioid receptors. Life Sciences, 75(5), 511-536.

Mansour, Fox, Akil, \& Watson. (1995). Opioid-receptor mRNA expression in the rat CNS: anatomical and functional implications. Trends in Neurosciences, 18(1), 22-29.

McClung, \& Nestler. (2003). Regulation of gene expression and cocaine reward by CREB and $\triangle$ FosB. Nature Neuroscience, 6(11), 1208-1215.

McCurdy, Sufka, Smith, Warnick, \& Nieto. (2006). Antinociceptive profile of salvinorin A, a structurally unique kappa opioid receptor agonist. Pharmacology, Biochemistry and Behaviour, 83(1), 109-113. 
McLennan, Kiss, Miyatake, Belcheva, Chambers, Pozek, . . Coscia. (2008). Kappa

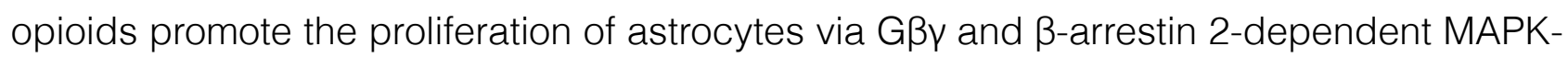
mediated pathways. Journal of Neurochemistry, 107(6), 1753-1765.

Institute of Medicine. (2011). Relieving pain in America: A blueprint for transforming prevention, care, education, and research. Washington, DC.

Mei, Mayoral, Nobuta, Wang, Desponts, Lorrain, ... Chan. (2016). Identification of the Kappa-Opioid Receptor as a Therapeutic Target for Oligodendrocyte Remyelination. Journal of Neuroscience, 36(30), 7925-7935.

Mello, \& Negus. (2000). Interactions between kappa opioid agonists and cocaine: preclinical studies. Annals of the New York Academy of Sciences, 909(1), 104-132.

Mendez, Arias, Uceda, \& Arias. (2015). c-Fos expression correlates with performance on novel object and novel place recognition tests. Brain Research Bulletin, 117, 16-23.

Mochizuki-Kawai. (2008). Neural basis of procedural memory. Brain and nerveShinkei kenkyu no shinpo, 60(7), 825-832.

Montag-Sallaz, Welzl, Kuhl, Montag, \& Schachner. (1999). Novelty-induced increased expression of immediate-early genes c-fos and arg 3.1 in the mouse brain. Journal of Neurobiology, 38(2), 234-246.

Morani, Ewald, Prevatt-Smith, Prisinzano, \& Kivell. (2013). The 2-methoxy methyl analogue of salvinorin A attenuates cocaine-induced drug seeking and sucrose reinforcements in rats. European Journal of Pharmacology, 720(1-3), 69-76.

Morani, Schenk, Prisinzano, \& Kivell. (2012). A single injection of a novel kappa opioid receptor agonist salvinorin A attenuates the expression of cocaine-induced behavioral sensitization in rats. Behavioural Pharmacology, 23(2), 162-170.

Morris. (1984). Developments of a water-maze procedure for studying spatial learning in the rat. Journal of Neuroscience Methods, 11(1), 47-60.

Mucha, \& Herz. (1985). Motivational properties of kappa and mu opioid receptor agonists studied with place and taste preference conditioning. Psychopharmacology, 86(3), 274-280.

Muigg, Hoelzl, Palfrader, Neumann, Wigger, Landgraf, \& Singewald. (2007). Altered brain activation pattern associated with drug-induced attenuation of enhanced 
depression-like behavior in rats bred for high anxiety. Biological Psychiatry, 61(6), 782-796.

Murray, \& Mishkin. (1986). Visual recognition in monkeys following rhinal cortical ablations combined with either amygdalectomy or hippocampectomy. Journal of Neuroscience, 6(7), 1991-2003.

Nadel, \& Hardt. (2011). Update on memory systems and processes. Neuropsychopharmacology, 36(1), 251-273.

Nagase, Kawamura, Kawai, \& Hayakawa. (2011). Discovery of the First in Class Drug for Intractable Itch, Nalfurafine Hydrochloride. ChemInform, 42(31).

Nakao, \& Mochizuki. (2009). Nalfurafine hydrochloride: a new drug for the treatment of uremic pruritus in hemodialysis patients. Drugs of Today (Barcelona, Spain: 1998), 45(5), 323-329.

Narita, Kuzumaki, Miyatake, Sato, Wachi, Seyama, \& Suzuki. (2006). Role of $\delta$-opioid receptor function in neurogenesis and neuroprotection. Journal of Neurochemistry, 97(5), 1494-1505.

National Committee for Addiction Treatment. (2011). Addiction treatment is everybody's business.

National Committee for Addiction Treatment. (2016). Shaping the sector.

Onaolapo, Onaolapo, Akanmu, \& Olayiwola. (2015). Caffeine/sleep-deprivation interaction in mice produces complex memory effects. Annals of Neurosciences, 22(3), 139.

Owen, \& Brenner. (2012). Mapping molecular memory: navigating the cellular pathways of learning. Cellular and Molecular Neurobiology, 32(6), 919-941.

Paris, Reilley, \& McLaughlin. (2011). Kappa opioid receptor-mediated disruption of novel object recognition: Relevance for psychostimulant treatment. Journal of Addiction Research \& Therapy, S4.

Paton, Kumar, Crowley, Harper, Prisinzano, \& Kivell. (2017). The analgesic and antiinflammatory effects of Salvinorin A analogue beta-tetrahydropyran Salvinorin B in mice. European Journal of Pain.

Perez, \& Karnik. (2005). Multiple signaling states of G-protein-coupled receptors. Pharmacological Reviews, 57(2), 147-161. 
Pfeiffer, Brantl, Herz, \& Emrich. (1986). Psychotomimesis mediated by k opiate receptors. Science, 233, 774-777.

Phillips, \& LeDoux. (1992). Differential contribution of amygdala and hippocampus to cued and contextual fear conditioning. Behavioral Neuroscience, 106(2), 274.

Prisinzano, Tidgewell, \& Harding. (2005). k Opioids as potential treatments for stimulant dependence. The AAPS journal, 7(3), E592-E599.

Renthal, \& Nestler. (2009). Chromatin regulation in drug addiction and depression. Dialogues in Clinical Neuroscience, 11(3), 257.

Riley, Groer, Young, Ewald, Kivell, \& Prisinzano. (2014). Synthesis and kappa-opioid receptor activity of furan-substituted salvinorin A analogues. Journal of Medicinal Chemistry, 57(24), 10464-10475.

Robison, \& Nestler. (2011). Transcriptional and epigenetic mechanisms of addiction. Nature Reviews Neuroscience, 12(11), 623-637.

Roth, Baner, Westkaemper, Siebert, Rice, Steinberg, . . Rothman. (2002). Salvinorin A: a potent naturally occurring nonnitrogenous kappa opioid selective agonist. Proceedings of the National Academy of Sciences, 99(18), 11934-11939.

Rubaj, Gustaw, Zgodziński, Kleinrok, \& Sieklucka-Dziuba. (2000). The role of opioid receptors in hypoxic preconditioning against seizures in brain. Pharmacology Biochemistry and Behavior, 67(1), 65-70.

Rudd, Seth, David, \& Scholl. (2016). Increases in drug and opioid-involved overdose deaths - United States, 2010-2015. MMWR Morbidity and Mortality Weekly Report, 65(50-51), 1445-1452.

Rusin, Giovannucci, Stuenkel, \& Moises. (1997). K-opioid receptor activation modulates $\mathrm{Ca} 2+$ currents and secretion in isolated neuroendocrine nerve terminals. Journal of Neuroscience, 17(17), 6565-6574.

Saitoh, Yoshikawa, Onodera, \& Kamei. (2005). Role of $\delta$-opioid receptor subtypes in anxiety-related behaviors in the elevated plus-maze in rats. Psychopharmacology, 182(3), 327-334.

Sandi, \& Pinelo-Nava. (2007). Stress and memory: behavioral effects and neurobiological mechanisms. Neural Plasticity, 2007. 
Sandin, Nylander, Georgieva, Schött, Ögren, \& Terenius. (1998). Hippocampal dynorphin B injections impair spatial learning in rats: a k-opioid receptor-mediated effect. Neuroscience, 85(2), 375-382.

Saunders, Murray, \& Mishkin. (1984). Further evidence that amygdala and hippocampus contribute equally to recognition memory. Neuropsychologia, 22(6), 785-796.

Schenk, Partridge, \& Shippenberg. (1999). U69593, a kappa-opioid agonist, decreases cocaine self-administration and decreases cocaine-produced drug-seeking. Psychopharmacology, 144(4), 339-346.

Schindler, Li, \& Chavkin. (2010). Behavioral stress may increase the rewarding valence of cocaine-associated cues through a dynorphin/kappa-opioid receptormediated mechanism without affecting associative learning or memory retrieval mechanisms. Neuropsychopharmacology, 35(9), 1932-1942.

Schmidt, Schmidt, Butelman, Harding, Tidgewell, Murry, . . . Prisinzano. (2005). Pharmacokinetics of the plant-derived kappa-opioid hallucinogen salvinorin $A$ in nonhuman primates. Synapse, 58(3), 208-210.

Schmitz, \& Hof. (2005). Design-based stereology in neuroscience. Neuroscience, 130(4), 813-831.

Schroder, O'Dell, \& Marshall. (2003). Neurotoxic methamphetamine regimen severely impairs recognition memory in rats. Synapse, 49(2), 89-96.

Schwarzer. (2009). 30 years of dynorphins--new insights on their functions in neuropsychiatric diseases. Pharmacology \& Therapeutics, 123(3), 353-370.

Shaywitz, \& Greenberg. (1999). CREB: a stimulus-induced transcription factor activated by a diverse array of extracellular signals. Annual Review of Biochemistry, 68(1), 821-861.

Sheng, \& Greenberg. (1990). The regulation and function of c-fos and other immediate early genes in the nervous system. Neuron, 4(4), 477-485.

Shippenberg, Zapata, \& Chefer. (2007). Dynorphin and the pathophysiology of drug addiction. Pharmacology \& Therapeutics, 116(2), 306-321.

Shu, Wu, Bao, \& Leonard. (2003). Interactions among memory-related centers in the brain. Journal of Neuroscience Research, 71(5), 609-616. 
Siebert. (1994). Salvia divinorum and salvinorin A: new pharmacologic findings. Journal of Ethnopharmacology, 43(1), 53-56.

Silva, Kogan, Frankland, \& Kida. (1998). CREB and memory. Annual Review of Neuroscience, 21(1), 127-148.

Simonin, Valverde, Smadja, Slowe, Kitchen, Dierich, ... Kieffer. (1998). Disruption of the k-opioid receptor gene in mice enhances sensitivity to chemical visceral pain, impairs pharmacological actions of the selective $\mathrm{k}$-agonist $\mathrm{U}-50,488 \mathrm{H}$ and attenuates morphine withdrawal. The EMBO Journal, 17(4), 886-897.

Simonson, Morani, Ewald, Walker, Kumar, Simpson, . . Kivell. (2015b). Pharmacology and anti-addiction effects of the novel k opioid receptor agonist Mesyl Sal B, a potent and long-acting analogue of salvinorin A. British Journal of Pharmacology, 172(2), 515-531.

Strupp, \& Levitsky. (1984). Social transmission of food preferences in adult hooded rats (Rattus norvegicus). Journal of Comparative Psychology, 98(3), 257.

Sufka, Loria, Lewellyn, Zjawiony, Ali, Abe, \& Khan. (2014). The effect of Salvia divinorum and Mitragyna speciosa extracts, fraction and major constituents on place aversion and place preference in rats. Journal of ethnopharmacology, 151(1), 361-364.

Taussig, Iñiguez-Lluhi, \& Gilman. (1993). Inhibition of adenylyl cyclase by Gia. Science, 261, 218-218.

The European Dana Alliance for the Brain. (2003). Neuroscience, Science of the Brain. Liverpool: The British Neuroscience Association.

Tian, Zhou, Yang, Xia, Wu, \& Guo. (2008). Role of $\delta$-opioid receptors in cumulative electro-acupuncture induced protection from ischemic injury in the rat brain. Shanghai $J$ TCM, 42, 71-74.

Tischmeyer, \& Grimm. (1999). Activation of immediate early genes and memory formation. Cellular and Molecular Life Sciences, 55(4), 564-574.

Tully. (1991). Genetic dissection of learning and memory in Drosophila melanogaster. Neurobiology of Learning, Emotion and Affect, 30-66.

Tulving. (1986). Episodic and semantic memory: Where should we go from here? Behavioral and Brain Sciences, 9(03), 573-577. 
Ueno, Mori, \& Yanagita. (2013). One year long-term study on abuse liability of nalfurafine in hemodialysis patients. International Journal of Clinical Pharmacology and Therapeutics, 51(11), 823-831.

Urban, Clarke, Von Zastrow, Nichols, Kobilka, Weinstein, . . . Sexton. (2007). Functional selectivity and classical concepts of quantitative pharmacology. Journal of Pharmacology and Experimental Therapeutics, 320(1), 1-13.

van der Staay, Schuurman, van Reenen, \& Korte. (2009). Emotional reactivity and cognitive performance in aversively motivated tasks: a comparison between four rat strains. Behavioral and Brain Functions, 5(1), 50.

Vieira-Brock, McFadden, Nielsen, Smith, Hanson, \& Fleckenstein. (2015). Nicotine Administration Attenuates Methamphetamine-Induced Novel Object Recognition Deficits. International Journal of Neuropsychopharmacology, 18(12).

Vonvoigtlander, Lahti, \& Ludens. (1983). U-50,488: a selective and structurally novel non-Mu (kappa) opioid agonist. Journal of Pharmacology and Experimental Therapeutics, 224(1), 7-12.

Wang, Sun, Tao, Chi, \& Liu. (2010). The role of kappa-opioid receptor activation in mediating antinociception and addiction. Acta Pharmacologica Sinica, 31(9), 1065-1070.

Warburton, Glover, Massey, Wan, Johnson, Bienemann, . . . Bashir. (2005). cAMP responsive element-binding protein phosphorylation is necessary for perirhinal long-term potentiation and recognition memory. Journal of Neuroscience, 25(27), 6296-6303.

Warburton, Koder, Cho, Massey, Duguid, Barker, . . B Brown. (2003). Cholinergic neurotransmission is essential for perirhinal cortical plasticity and recognition memory. Neuron, 38(6), 987-996.

West, Slomianka, \& Gundersen. (1991). Unbiased Stereological Estimation of the Total Number of Neurons in the Subdivisions of the Rat Hippocampus Using the Optical Fractionator. Anatomical Record, 231(4), 482-497.

Wilkinson, Herrman, Palmatier, \& Bevins. (2006). Rats' novel object interaction as a measure of environmental familiarity. Learning and Motivation, 37(2), 131-148.

Winters, Saksida, \& Bussey. (2008). Object recognition memory: neurobiological mechanisms of encoding, consolidation and retrieval. Neuroscience and Biobehavioural Reviews, 32(5), 1055-1070. 
Wisler, Xiao, Thomsen, \& Lefkowitz. (2014). Recent developments in biased agonism. Current Opinion in Cell Biology, 27, 18-24.

Wittert, Hope, \& Pyle. (1996). Tissue distribution of opioid receptor gene expression in the rat. Biochemical and Biophysical Research Communications, 218(3), 877-881.

Wu, Li, \& Wong. (1999). Cardioprotection of preconditioning by metabolic inhibition in the rat ventricular myocyte. Circulation Research, 84(12), 1388-1395.

Wyss, Chambless, Kadish, \& van Groen. (2000). Age-related decline in water maze learning and memory in rats: strain differences. Neurobiology of Aging, 21(5), 671-681.

Yin, Del Vecchio, Zhou, \& Tully. (1995). CREB as a memory modulator: induced expression of a dCREB2 activator isoform enhances long-term memory in Drosophila. Cell, 81(1), 107-115.

Yin, \& Tully. (1996). CREB and the formation of long-term memory. Current Opinion in Neurobiology, 6(2), 264-268.

Yin, Wallach, Del Vecchio, Wilder, Zhou, Quinn, \& Tully. (1994). Induction of a dominant negative CREB transgene specifically blocks long-term memory in Drosophila. Cell, 79(1), 49-58.

Zan, Wang, Wang, Liu, Hang, Shu, \& Liu. (2015). Antagonism of kappa opioid receptor in the nucleus accumbens prevents the depressive-like behaviors following prolonged morphine abstinence. Behavioural Brain Research, 291, 334-341.

Zhu, Brown, McCabe, \& Aggleton. (1995). Effects of the novelty or familiarity of visual stimuli on the expression of the immediate early gene c-fos in rat brain. Neuroscience, 69(3), 821-829.

Zola-Morgan, \& Squire. (1985). Medial temporal lesions in monkeys impair memory on a variety of tasks sensitive to human amnesia. Behavioural Neuroscience, 99(1), 22-34.

Zola-Morgan, Squire, \& Amaral. (1989). Lesions of the amygdala that spare adjacent cortical regions do not impair memory or exacerbate the impairment following lesions of the hippocampal formation. Journal of Neuroscience, 9(6), 1922-1936.

Zola-Morgan, Squire, Clower, \& Rempel. (1993). Damage to the perirhinal cortex exacerbates memory impairment following lesions to the hippocampal formation. Journal of Neuroscience, 13(1), 251-265. 
Zuberi, Townsend, Patterson, Zheng, \& Berthoud. (2008). Increased adiposity on normal diet, but decreased susceptibility to diet-induced obesity in $\mu$-opioid receptordeficient mice. European Journal of Pharmacology, 585(1), 14-23. 